\title{
EISENSTEIN SERIES AND THE SELBERG TRACE FORMULA. II
}

\author{
H. JACQUET AND D. ZAGIER
}

\begin{abstract}
The integral of the kernel of the trace formula against an Eisenstein series is investigated. The analytic properties of this integral imply the divisibility of the convolution $L$-function attached to a form by the zeta function of the field.
\end{abstract}

Introduction. This paper is a sequel and generalization of [12], but can be read independently of that paper; in particular, we will repeat the description of the problem given in the introduction of [12], now, however, in an adelic setting.

Let $F$ be a global field, $A$ its ring of adeles, and $\rho_{0}$ the representation of $\operatorname{PGL}(2, A)$ by right translation on the space of cusp forms $L_{0}^{2}(\operatorname{PGL}(2, F) \backslash$ $\operatorname{PGL}(2, \mathbf{A}))$. Given any $\varphi \in C_{0}^{\infty}(\operatorname{PGL}(2, \mathbf{A}))$, the operator $\rho_{0}(\varphi)$ on this space is of Hilbert-Schmidt type and can be represented by a kernel function $K_{0}(x, y)$ for which an explicit formula of the form

$$
K_{0}(x, y)=K(x, y)-K_{\text {Eis }}(x, y)-K_{\text {sp }}(x, y)
$$

is known, where $K$ is given as a sum over $\operatorname{PGL}(2, F), K_{\mathrm{Eis}}$ as an integral involving Eisenstein series, and $K_{\mathrm{sp}}$ as a sum of products of characters. In particular, one can calculate $\operatorname{tr} \rho_{0}(\varphi)$ from the identity

$$
\operatorname{tr} \rho_{0}(\varphi)=\int_{\mathrm{PGL}(2, F) \backslash \mathrm{PGL}(2, \mathbf{A})} K_{0}(x, x) d x ;
$$

the result is the Selberg trace formula.

What we will do is to calculate instead the integral

$$
I(s)=\int_{\mathrm{PGL}(2, F) \backslash \mathrm{PGL}(2, \mathbf{A})} K_{0}(x, x) E(x, s) d x,
$$

where $E(x, s)$ is an Eisenstein series. Our main result is an identity expressing $I(s)$, roughly speaking, as a finite linear combination of zeta functions of quadratic extensions of $F$. Since the residue of $E(x, s)$ at $s=1$ is a constant function of $x$, one can in principle recover the Selberg trace formula from this identity by computing the residue of $I(s)$ at $s=1$, but the formula for $I(s)$ has other interesting consequences. Most notably, it implies that, as in the special cases treated in [11 and 12], $I(s)$ is divisible by $\zeta_{F}(s)$ or, in other words, that the function $K_{0}(x, x)$ for any $\varphi$ is orthogonal to the functions $E(x, \rho)$ for all zeros $\rho$ of the zeta function of $F$. A somewhat more precise formulation of the main result is as follows.

Received by the editors January 11, 1986.

1980 Mathematics Subject Classification (1985 Revision). Primary 11F70; Secondary 11R39. 
THEOREM. For $0<\mathscr{R}_{e}(s)<1$,

$$
I(s)=\sum_{E} I_{E}(s)+\sum_{i=1}^{5} I_{i}(s)+\sum_{\chi} \frac{1}{2 \pi i} \int_{\operatorname{Im}(u)=1 / 2} I_{\chi}(s, u) d u
$$

where the first sum is a finite sum over certain quadratic extensions $E$ of $F$, the last sum is a finite sum over certain grossencharacters of $F$, and

$$
\begin{aligned}
& I_{E}(s) \text { is a multiple of } \zeta_{E}(s), \\
& I_{1}(s) \text { is a multiple of } \zeta_{F}(s)^{2}, \\
& I_{2}(s) \text { is a multiple of } \zeta_{F}(s) \zeta_{F}(2 s-1), \\
& I_{3}(s) \text { is a multiple of } \zeta_{F}(s) \zeta_{F}(2 s), \\
& I_{4}(s) \text { is a multiple of } \zeta_{F}(s) \zeta_{F}(2 s) / \zeta_{F}(s+1), \\
& I_{5}(s) \text { is a multiple of } \zeta_{F}(s) \zeta_{F}(2 s-1) / \zeta_{F}(s-1), \\
& I_{\chi}(s, u) \text { is a multiple of } \zeta_{F}(s)^{2} \frac{L(s+1-2 u, \chi) L(s-1+2 u, \chi)}{L(2-2 u, \chi) L(2 u, \chi)} .
\end{aligned}
$$

(Here "multiple" means "product with an entire function".) The actual statement proved in the text will involve $L$-functions rather than zeta-functions, because $\varphi$ will be taken as a function on $\operatorname{GL}(2, A)$ with a given central character rather than a function on $\operatorname{PGL}(2, \mathrm{~A})$.

The organization of the paper is as follows. The first section, apart from a few lemmas (e.g. an estimate of the growth of Whittaker functions), is just a review of those facts from the theory of Eisenstein series which will be needed in the sequel; it can be skipped by the expert reader. The bulk of the paper is $\$ 2$, in which we calculate $I(s)$ for $\mathscr{R} e(s)>1$, obtaining the same formula as given above but without the term $I_{5}$. The extra term $I_{5}(s)$ in the strip $0<\mathscr{R} e(s)<1$ appears as a residue coming from the poles of $I_{\chi}(s, u)$ at $u=s / 2$ and $u=1-s / 2$ when $\chi$ is the trivial character. (For the same reason, the poles at $u=(1 \pm s) / 2$ give a contribution which cancels the term $I_{4}(s)$ when we cross the line $\mathscr{R} e(s)=0$. A similar phenomenon already occurred in [12].) This will be carried out in $\$ 3$, where we also give the main application - the divisibility of $I(s)$ by $\zeta_{F}(s)$, and as a consequence of this the holomorphy of the symmetric square of the $L$-series attached to a cusp form. This latter fact was proved for classical holomorphic forms by Shimura [10] and independently by one of the authors [11]; Shimura's method was generalized by Gelbart and Jacquet [3] to an adelic setting, while the present paper is essentially the adelic generalization of [11]. Another application is-or should be-the trace formula, which as explained above arises by calculating the residue of $I(s)$ at $s=1$. This calculation is complicated by the fact that some of the terms in the formula for $I(s)$ (namely the terms $I_{1}, I_{2}$ and $I_{\chi}$ in the theorem above) have double poles at $s=1$, and although the coefficients of $(s-1)^{-2}$ naturally cancel in the sum, this means that we need two terms of the Laurent expansion rather than just the leading term in order to calculate the residue. We were able to calculate the residues of $I_{E}$ and $I_{1}$ to $I_{5}$ explicitly, obtaining six of the seven terms in the usual adelic Selberg trace 
formula (cf. 3.2), but the formula for $I_{\chi}(s, u)$ is so complicated that we could not reduce the expression for its residue to the corresponding term in the trace formula as it is usually formulated. After almost a five year delay during which we hoped to return to and settle this point, we decided to publish the paper with the deduction of the usual trace formula from our formula left incomplete.

In any case, both of these two consequences of our theorem-the trace formula and the holomorphy of symmetric square $L$-functions for representations of GL(2) - were already known. The main interest in our result lies in the form of the identity, which can be thought of as a generalization of the trace formula in which the various terms are expressed locally (i.e. as products of local integrals). Furthermore, the method can in principle be generalized to GL( $n)$. For GL(3) this has already been partially carried out by Parameswaran Kumar.

0. Notations and conventions. Tate integrals. $F$ denotes a global field, $F_{v}$ the completion of $F$ at a typical place $v, R_{v}$ the valuation ring of $F_{v}$, and A and $\mathrm{A} \times$ the adeles and ideles of $F$. We will generally use Greek letters for elements of $F$ and Latin letters for adelic variables. The norm map from $A^{\times}$to $\mathbb{R}_{+}^{\times}$is denoted by || , the set of ideles of norm 1 by $\mathbf{A}_{1}^{\times}$. We choose once and for all a splitting $\mathbf{A}^{\times}=\mathbf{A}_{1}^{\times} \times \mathbb{R}_{+}^{\times}$ and denote by $\Lambda$ the set of characters on $A^{\times} / F^{\times}$which are trivial on $\mathbb{R}_{+}^{\times}$; thus the most general (quasi-) character on $\mathbf{A}^{\times} / F^{\times}$has the form $a \mapsto \chi(a)|a|^{s}$ with $\chi \in \Lambda$, $s \in \mathbb{C}$. We choose once and for all a nontrivial additive character $\psi: \mathbb{A} / F \rightarrow \mathbb{C}$. The Haar measures on $\mathbf{A}, \mathbf{A}_{1}^{\times}$and $\mathbf{A}^{\times}$are normalized by $\int_{\mathbf{A} / F} d x=1, \int_{\mathbf{A}_{1}^{\times} / F^{\times}} d^{\times} a_{1}=1$ and $d^{\times} a=d^{\times} a_{1} \times d^{\times} t$, where $d^{\times} t=d t / t$ is the standard Haar measure on $\mathbb{R}_{+}^{\times}$.

We denote by $G$ the algebraic group GL(2) and by $Z, A, N$ and $P(=A N)$ the subgroups of matrices of the form $\left(\begin{array}{ll}a & 0 \\ 0 & a\end{array}\right),\left(\begin{array}{ll}a & 0 \\ 0 & b\end{array}\right),\left(\begin{array}{ll}1 & x \\ 0 & 1\end{array}\right)$ and $\left(\begin{array}{cc}a & x \\ 0 & b\end{array}\right)$, respectively; we write $G_{F}, G_{v}$ and $G_{\mathrm{A}}$ for $G(F), G\left(F_{v}\right)$ and $G(\mathrm{~A})$ and similarly for the other groups. We denote by $K=\Pi_{v} K_{v}$ the standard maximal compact subgroup of $G_{\mathrm{A}}$. We will often identify $N_{\mathbf{A}}, Z_{\mathbf{A}}$ and $A_{\mathbf{A}}$ with $\mathbf{A}, \mathbf{A}^{\times}$and $\left(\mathbf{A}^{\times}\right)^{2}$, respectively; in particular, this will be done to define the Haar measures on these groups and also to identify $\psi$, elements of $\Lambda$, and pairs of elements of $\Lambda$ with characters of $N_{\mathbf{A}} / N_{F}$, of $Z_{\mathbf{A}} / Z_{F}$, and of $A_{\mathrm{A}} / A_{F}$, respectively. The Haar measure on $K$ is normalized so that $\int_{K} d k=1$ and the Haar measure on $G_{\mathbf{A}}$ then chosen so that

$$
\int_{G_{\mathbf{A}}} f(g) d g=\int_{K} \int_{A_{\mathbf{A}}} \int_{N_{\mathbf{A}}} f(k n a) d n d a d k=\int_{K} \int_{A_{\mathbf{A}}} \int_{N_{\mathbf{A}}} f(k a n)\left|a_{2} / a_{1}\right| d n d a d k
$$

(here $a_{1}, a_{2}$ denote the diagonal components of $a \in \mathbf{A}$ ). We denote by $w$ the element $\left(\begin{array}{ll}0 & 1 \\ 1 & 0\end{array}\right)$ of $G$ and by $g \mapsto g^{\imath}(g \in G)$ the involution $g \mapsto^{t} g^{-1}$.

We denote by $\mathscr{S}(\mathbf{A})$ the space of Schwartz-Bruhat functions and by $\mathscr{S}_{0}(\mathbf{A})$ the subspace spanned by products $\Phi=\Pi_{v} \Phi_{v}$ whose components at real and complex places have the form

$$
\begin{array}{ll}
\Phi_{v}\left(x_{v}\right)=e^{-\pi x_{v}^{2}} \times \text { polynomial in } x_{v} & (v \text { real }), \\
\Phi_{v}\left(x_{v}\right)=e^{-2 \pi x_{v}, \bar{x}_{v}} \times \text { polynomial in } x_{v}, \bar{x}_{v} & (v \text { complex })
\end{array}
$$


the spaces $\mathscr{S}\left(\mathrm{A}^{2}\right)$ and $\mathscr{S}_{0}\left(\mathrm{~A}^{2}\right)$ are defined analogously. We denote by

$$
\begin{array}{ll}
\hat{\Phi}(x)=\int_{\mathbf{A}} \Phi(u) \psi(x u) d u & (\Phi \in \mathscr{S}(\mathbf{A})), \\
\hat{\Phi}(x, y)=\int_{\mathbf{A}} \int_{\mathbf{A}} \Phi(u, v) \bar{\psi}(x u+y v) d u d v & \left(\Phi \in \mathscr{S}\left(\mathbf{A}^{2}\right)\right), \\
\tilde{\Phi}(x, y)=\int_{\mathbf{A}} \Phi(x, v) \bar{\psi}(y v) d v & \left(\Phi \in \mathscr{S}\left(\mathbf{A}^{2}\right)\right),
\end{array}
$$

the Fourier transform and Fourier transform with respect to the second variable. For $g \in G_{\mathbf{A}}$ and $\Phi \in \mathscr{S}\left(\mathbf{A}^{2}\right), g \Phi$ denotes the function $g \Phi(x, y)=\Phi[(x, y) g]$; thus $\widehat{g \Phi}=|\operatorname{det} g|^{-1} g^{\imath} \hat{\Phi}$. Finally, we call any integral of the form

$$
\int_{\mathbf{A}^{\times}} \Phi(a) \chi(a)|a|^{s} d^{\times} a \quad(\Phi \in \mathscr{S}(\mathbb{A}), \chi \in \Lambda, s \in \mathbb{C}, \mathscr{R} e(s)>1)
$$

a Tate integral for $L(s, \chi)$ (where $L(s, \chi)$ itself is defined by making the appropriate standard choice for $\Phi)$ and denote by $L(\Phi, s, \chi)$ the meromorphic continuation of this function, i.e.

$$
\begin{aligned}
L(\Phi, s, \chi)= & L(\hat{\Phi}, 1-s, \bar{\chi}) \\
= & \int_{|a| \geqslant 1} \Phi(a) \chi(a)|a|^{s} d^{\times} a+\int_{|a| \geqslant 1} \hat{\Phi}(a) \bar{\chi}(a)|a|^{1-s} d^{\times} a \\
& + \begin{cases}\frac{\hat{\Phi}(0)}{s-1}-\frac{\Phi(0)}{s} & \text { if } \chi=1, \\
0 & \text { otherwise. }\end{cases}
\end{aligned}
$$

Finally, we mention the identity

$$
\int_{K} \int_{\mathbf{A}^{\times}} \Phi[(0, a) k]|a|^{2} d^{\times} a d k=c \hat{\Phi}(0) \quad\left(\Phi \in \mathscr{S}\left(\mathbf{A}^{2}\right)\right),
$$

which can be thought of as the analogue of the ordinary polar coordinates formula

$$
\int_{-\infty}^{\infty} \int_{-\infty}^{\infty} \Phi(x+i y) d x d y=\int_{0}^{2 \pi} \int_{0}^{\infty} \Phi\left[r e^{i \theta}\right] r^{2} \frac{d r}{r} d \theta
$$

in $\mathbf{R}^{2}$. The constant $c$ is given by

$$
c=\frac{L\left(2,1_{F}\right)|D|^{1 / 2}}{\operatorname{Res}_{s=1} L\left(s, 1_{F}\right)},
$$

where $D$ is the discriminant and $L\left(s, 1_{F}\right)$ the zeta-function (with factors at infinity added) of $F$. It is also equal to $\frac{1}{2} \operatorname{vol}\left(Z_{\mathbf{A}} G_{F} \backslash G_{\mathbf{A}}\right)$.

1. Review of Eisenstein series and related topics. Almost all of the material in this section is standard; we refer the reader to [2, 4].

1.1. Eisenstein series. For $\chi_{1}, \chi_{2} \in \Lambda$ and $S \in \mathbb{C}$ we denote by $\pi_{\chi_{1}, \chi_{2}, s}$ the representation of $G_{\mathbf{A}}$ by right translation on the space $H\left(\chi_{1}, \chi_{2}, s\right)$ of (classes of) functions $f$ on $G_{\mathbf{A}}$ satisfying

$$
f\left[\left(\begin{array}{ll}
a & x \\
0 & b
\end{array}\right) g\right]=\chi_{1}(a) \chi_{2}(b)\left|\frac{a}{b}\right|^{s} f(g) \quad\left(a, b \in \mathbf{A} \times, x \in \mathbf{A}, g \in G_{\mathbf{A}}\right)
$$


and

$$
\int_{K}|f(k)|^{2} d k<\infty
$$

Because of the Iwasawa decomposition, the functions in $H\left(\chi_{1}, \chi_{2}, s\right)$ are determined by their restrictions to $K$, so we may identify all these spaces with the space

$$
\begin{aligned}
H\left(\chi_{1}, \chi_{2}\right)=\left\{f \in L^{-}(K) \mid f\left[\left(\begin{array}{ll}
a & x \\
0 & b
\end{array}\right) k\right]\right. & =\chi_{1}(a) \chi_{2}(b) f(k) \\
\forall k & \left.\in K,\left(\begin{array}{ll}
a & x \\
0 & b
\end{array}\right) \in P \cap K\right\} .
\end{aligned}
$$

In other words, $\bigcup_{s \in \mathbb{C}} H\left(\chi_{1}, \chi_{2}, s\right)$ is a fiber bundle over $\mathbf{C}$ and we have trivialized this bundle. Given any $f \in H\left(\chi_{1}, \chi_{2}\right)$, the corresponding section of $\cup_{s} H\left(\chi_{1}, \chi_{2}, s\right)$ is defined by

$$
S f\left[\left(\begin{array}{cc}
a & x \\
0 & b
\end{array}\right) k, s\right]=\chi_{1}(a) \chi_{2}(b)\left|\frac{a}{b}\right|^{s} f(k) \quad\left(a, b \in \mathbf{A}^{\times}, x \in \mathbf{A}, k \in K\right)
$$

and the corresponding Eisenstein series (for $\mathscr{R} e(s)>1$ ) by

$$
E f(g, s)=\sum_{\gamma \in P_{F} \backslash G_{F}} S f(\gamma g, s) \quad\left(g \in G_{\mathbf{A}}\right) .
$$

For later purposes, we fix once and for all an orthonormal basis (of $K$-finite functions) $\left\{f_{\alpha}\right\}_{\alpha \in A\left(\chi_{1}, \chi_{2}\right)}$ of the Hilbert space $H\left(\chi_{1}, \chi_{2}\right)$; the corresponding functions $S f_{\alpha}(g, s)$ and $E f_{\alpha}(g, s)$ will sometimes be denoted simply $S_{\alpha}(g, s), E_{\alpha}(g, s)$.

The definition of Eisenstein series just given corresponds to the classical series

$$
E(z, s)=\sum_{\gamma \in\left\{ \pm\left(\begin{array}{c}
1 \\
0 \\
1
\end{array}\right)\right\} \backslash \operatorname{SL}(2, \mathbf{z})} \operatorname{Im}(\gamma z)^{s} \quad(z, s \in \mathbb{C}, \operatorname{Im}(z)>0, \mathscr{R} e(s)>1) .
$$

For analytic purposes another definition, analogous to the function

$$
\frac{1}{2} \operatorname{Im}(z)^{s} \sum_{(m, n) \in \mathbf{Z}^{2}-0}(m z+n)^{-2 s}=\zeta(2 s) E(z, s),
$$

is more convenient: For $\Phi \in S\left(\mathrm{~A}^{2}\right)$ the function

$$
f\left(g, \Phi, \chi_{1}, \chi_{2}, s\right)=\chi_{1}(\operatorname{det} g)|\operatorname{det} g|^{s} \int_{\mathbf{A}^{\times}} \Phi[(0, t) g] \chi_{1} \chi_{2}^{-1}(t)|t|^{2 s} d^{\times} t
$$

(which is a Tate integral for $\left.L\left(2 s, \chi_{1} \chi_{2}^{-1}\right)\right)$ belongs to $H\left(\chi_{1}, \chi_{2}, s\right)$ and the corresponding Eisenstein series

$$
E(g, s)=E\left(g, \Phi, \chi_{1}, \chi_{2}, s\right)=\sum_{\gamma \in P_{F} \backslash G_{F}} f\left(\gamma g, \Phi, \chi_{1}, \chi_{2}, s\right),
$$

again convergent for $\mathscr{R} e(s)>1$, can be rewritten as

$$
E(g, s)=\chi_{1}(\operatorname{det} g)|\operatorname{det} g|^{s} \int_{\mathbf{A}^{\times} / F^{\times}} \sum_{\xi \in F^{2}-0} \Phi[\xi t g]|t|^{2 s} \chi_{1} \chi_{2}^{-1}(t) d^{\times} t .
$$

The Poisson summation formula now implies that $E(g, s)$ has a meromorphic continuation to all $s$, satisfies the functional equation

$$
E\left(g, \Phi, \chi_{1}, \chi_{2}, s\right)=E\left(g^{\iota}, \hat{\Phi}, \chi_{1}^{-1}, \chi_{2}^{-1}, 1-s\right)
$$


(see "Notations and Conventions"), and is holomorphic unless $\chi_{1}=\chi_{2}$, when it has simple poles at $s=0$ and $s=1$ with residues given by

$$
\operatorname{Res}_{s=1} E(g, s)=\frac{1}{2} \hat{\Phi}(0) \chi_{1}(\operatorname{det} g), \quad \operatorname{Res}_{s=0} E(g, s)=-\frac{1}{2} \Phi(0) \chi_{1}(\operatorname{det} g) .
$$

The relationship between the two types of Eisenstein series is given by the following lemma.

Lemma. If $f \in H\left(\chi_{1}, \chi_{2}\right)$ is $K$-finite, then the section $S f(g, s)$ can be written as a finite linear combination

$$
S f(g, s)=L\left(2 s, \chi_{1} \chi_{2}^{-1}\right)^{-1} \sum_{i} P_{i}(s) f\left(g, \Phi_{i}, \chi_{1}, \chi_{2}, s\right)
$$

and correspondingly the Eisenstein series $E_{j}(g, s)$ as

$$
E f(g, s)=L\left(2 s, \chi_{1} \chi_{2}\right)^{-1} \sum_{i} P_{i}(s) E\left(g, \Phi_{i}, \chi_{1}, \chi_{2}, s\right),
$$

where $\Phi_{i} \in S_{0}\left(\mathbf{A}^{2}\right)$ and each $P_{i}(s)$ is the reciprocal of a polynomial in $s$ and in $q_{v}^{-s}$ for finitely many places $v$ which has no zeroes in the half-plane $\mathscr{R}_{e}(s)>0$.

Proof. The space $H\left(\chi_{1}, \chi_{2}\right)$ is the restricted tensor product over all places $v$ of analogously defined spaces $H_{v}\left(\chi_{1 v}, \chi_{2 v}\right)$, and we may assume that $f=\prod_{v} f_{v}$ with $f_{v} \in H_{v}\left(\chi_{1 v}, \chi_{2 v}\right), f_{v} \equiv 1$ for almost all finite $v$. We claim that for such an $f$ we can write (with the obvious notations)

$$
S_{v} f_{v}\left(g_{v}, s\right)=\frac{P_{v}(s)}{L_{v}\left(2 s, \chi_{1 v} \chi_{2 v}^{-1}\right)} f_{v}\left(g_{v}, \Phi_{v}, \chi_{1 v}, \chi_{2 v}, s\right)
$$

where $\Phi_{v}$ is a Schwartz-Bruhat function on $F_{v}^{2}$, equal to the characteristic function of $R_{v}^{2}$ for almost all $v$, and $P_{v}(s)$ is an elementary function of $s$, equal to 1 for almost all $v$. From this it will follow that $f(g, s)$ equals

$$
P(s) L\left(2 s, \chi_{1} \chi_{2}^{-1}\right)^{-1} f\left(g, \Phi, \chi_{1}, \chi_{2}, s\right)
$$

with $P=\Pi P_{v}$ and $\Phi=\Pi \Phi_{v} \in \mathscr{S}\left(\mathbf{A}^{2}\right)$.

To prove (*) we must distinguish several cases. Observe that it suffices to check (*) for $g_{v} \in K_{v}$ since both sides belong to $H_{v}\left(\chi_{1 v}, \chi_{2 v}, s\right)$. For almost all finite places $v$, $f_{v}$ is identically 1 and $\chi_{1 v}$ and $\chi_{2 v}$ are unramified; then (*) holds with $P_{v} \equiv 1$ and $\Phi_{v}$ the characteristic function of $R_{v}^{2}$. For the remaining finite places $v$ we define

$$
\Phi_{v}(x, y)= \begin{cases}f_{v}(k) \chi_{1 v}^{-1}(\operatorname{det} k) & \text { if }(x, y)=(0,1) k, \quad k \in K_{v}, \\ 0 & \text { otherwise; }\end{cases}
$$

this is well-defined, and for $k \in K_{v}$ we have

$$
\begin{aligned}
f_{v}\left(k, \Phi_{v}, \chi_{1 v}, \chi_{2 v}, s\right) & =\chi_{1 v}(\operatorname{det} k) \int_{R_{v}^{\times}} \Phi_{v}[(0, t) k] \chi_{1 v} \chi_{2 v}^{-1}(t)|t|_{v}^{2 s} d^{\times} t \\
& =f_{v}(k),
\end{aligned}
$$

and therefore $(*)$ holds with $P_{v}(s)=L_{v}\left(2 s, \chi_{1 v} \chi_{2 v}^{-1}\right)$, which is the reciprocal of a polynomial in $q_{v}^{-s}$ having no zeroes in $\mathscr{R} e(s)>0$. If $v$ is a real place, then (since $f$ is $K$-finite) we may assume that $f_{v}$ has the form

$$
f_{v}\left(\begin{array}{cc}
\cos \theta & \sin \theta \\
-\sin \theta & \cos \theta
\end{array}\right)=e^{i n \theta}
$$


for some $n \in \mathbf{Z}$ such that $\chi_{1 v} \chi_{2 v}(-1)=(-1)^{n}$; then we set

$$
\Phi_{v}(x, y)=e^{-\pi\left(x^{2}+y^{2}\right)}(y-\varepsilon i x)^{|n|} \quad\left(x, y \in F_{v}\right)
$$

with $\varepsilon=\operatorname{sign}(n)$ and find

$$
\begin{aligned}
f_{v}\left[\left(\begin{array}{cc}
\cos \theta & \sin \theta \\
-\sin \theta & \cos \theta
\end{array}\right), \Phi_{v}, \chi_{1 v}, \chi_{2 v}, s\right] & =e^{i n \theta} \int_{\mathbf{R}^{\times}} e^{-\pi t^{2} t^{|n|}|t|^{2 s} \chi_{1} \chi_{2}^{-1}(t) d^{\times} t} \\
& =Q(s) L\left(2 s, \chi_{1} \chi_{2}^{-1}\right) f_{v}\left[\left(\begin{array}{cc}
\cos \theta & \sin \theta \\
-\sin \theta & \cos \theta
\end{array}\right)\right]
\end{aligned}
$$

where $Q(s)$ is a polynomial whose roots lie in the half-plane $\mathscr{R}_{e}(s) \leqslant 0$, so $(*)$ holds with $P_{v}(s)=Q(s)^{-1}$. Finally, let $v$ be a complex plane. Then $\chi_{1 v} \chi_{2 v}^{-1}(z)=$ $(z /|z|)^{a}|z|^{i x}$ for some $x \in \mathbb{R}, a \in \mathbb{Z}$ and all $z \in F_{v}^{\times}$. Say $a \geqslant 0$. We may assume that $f$ has the form

$$
f\left(\begin{array}{cc}
\bar{\alpha} & -\bar{\beta} \\
\beta & \alpha
\end{array}\right)=\alpha^{p} \bar{\alpha}^{q} \beta^{r} \bar{\beta}^{u}
$$

with $p, q, r, u \in \mathbb{Z}$ and $q+u=p+r+a$. Then we take

$$
\Phi_{v}(x, y)=x^{r} \bar{x}^{u} y^{p} \bar{y}^{q} e^{-2 \pi(x \bar{x}+y \bar{y})}
$$

and again find

$$
\begin{aligned}
f_{v}\left[\left(\begin{array}{cc}
\bar{\alpha} & -\bar{\beta} \\
\beta & \alpha
\end{array}\right), \Phi_{v}, \chi_{1 v}, \chi_{2 v}, s\right] & =\alpha^{p} \bar{\alpha}^{q} \beta^{r} \bar{\beta}^{u} \int_{\mathbb{C}^{\times}} t^{p+r} \bar{t}^{q+u} t^{a}|t|^{2 s+i x} e^{-2 \pi|t|^{2}} d^{\times} t \\
& =Q(s) L\left(2 s, \chi_{1} \chi_{2}^{-1}\right) f_{v}\left[\left(\begin{array}{cc}
\bar{\alpha} & -\bar{\beta} \\
\beta & \alpha
\end{array}\right)\right]
\end{aligned}
$$

with $Q(s)$ a polynomial not vanishing for $\mathscr{R} e(s)>0$, so we can set $P_{v}(s)=Q(s)^{-1}$ as before. This completes the proof of the lemma.

Notation. For our standard basis elements $f_{\alpha}\left(\alpha \in \mathbf{A}\left(\chi_{1}, \chi_{2}\right)\right)$ we will sometimes use the notations $P_{\alpha, i}, \Phi_{\alpha, i}\left(i \in I_{\alpha}\right)$ for the polynomials and Schwartz-Bruhat functions occurring in the decomposition of $S_{\alpha}(g, s)$ given by the lemma.

1.2. Whittaker functions. We now discuss the Fourier coefficients of the Eisenstein series. The Bruhat decomposition gives for $\mathscr{R} e(s)>1$

$$
E f(g, s)=S f(g, s)+\sum_{\nu \in N_{F}} S f(w \nu g, s)
$$

and hence

$$
\int_{N_{F} \backslash N_{\mathbf{A}}} E f(n g, s) \bar{\psi}(n) d n=\int_{N_{\mathbf{A}}} S f(w n g, s) \bar{\psi}(n) d n,
$$

where we have used the isomorphism $x \mapsto\left(\begin{array}{ll}1 & x \\ 0 & 1\end{array}\right)$ between $\mathbf{A} / F$ and $N_{\mathbf{A}} / N_{F}$ to write $\psi$ as a character of the latter group. We will denote this function by $W f(g, s)$. It is a meromorphic function of $s$. To see how it depends on $s$, we apply the same construction to $E\left(g, \Phi, \chi_{1}, \chi_{2}, s\right)$ (which is sufficient because of the lemma in 1.1). 
We find

$$
\begin{aligned}
W\left(g, \Phi, \chi_{1}, \chi_{2}, s\right) & =\int_{N_{F} \backslash N_{\mathbf{A}}} E\left(n g, \Phi, \chi_{1}, \chi_{2}, s\right) \overline{\psi(n)} d n \\
& =\int_{N_{\mathbf{A}}} f\left(w n g, \Phi, \chi_{1}, \chi_{2}, s\right) \overline{\psi(n)} d n \\
& =\chi_{1}(\operatorname{det} g)|\operatorname{det} g|^{s} \int_{\mathbf{A}} \bar{\psi}(x) \int_{\mathbf{A} \times} \Phi[(t, t x) g] \chi_{1} \chi_{2}^{-1}(t)|t|^{2 s} d^{\times} t d x \\
& =\chi_{1}(\operatorname{det} g)|\operatorname{det} g|^{s} \int_{\mathbf{A}^{\times}} \widetilde{g \Phi}\left(t, t^{-1}\right) \chi_{1} \chi_{2}^{-1}(t)|t|^{2 s-1} d^{\times} t
\end{aligned}
$$

with $\widetilde{g \Phi}(x, y)$ as defined in $\S 0$. The last integral converges for all $s$, so $W\left(g, \Phi, \chi_{1}, \chi_{2}, s\right)$ is an entire function of $s$. We use it to obtain two further properties of Whittaker functions: an estimate of their growth and a formula for their Mellin transforms.

LEMMA 1. For any $\varepsilon>0, W\left(s, \Phi, \chi_{1}, \chi_{2}, s\right)$ satisfies

$$
\left|W\left(g, \Phi, \chi_{1}, \chi_{2}, s\right)\right| \leqslant \Phi_{0}(a)|a|^{1-\max \left(\mathscr{R e}(s), \mathscr{R}_{e}(1-s)\right)-\varepsilon}
$$

for $g=\left(\begin{array}{cc}a b & x \\ 0 & b\end{array}\right) k \in G_{\mathbf{A}}$, where $\Phi_{0}$ is a suitable Schwartz-Bruhat function on $\mathbf{A}$. Moreover, this estimate (i.e. the choice of $\Phi_{0}$ given $\Phi$ and $\varepsilon$ ) is uniform for $s$ in $a$ vertical strip.

Proof. Because $W$ is $K$-finite and invariant (up to a factor of absolute value 1 ) by $Z_{\mathbf{A}} N_{\mathbf{A}}$, we may assume that $g=\left(\begin{array}{ll}a & 0 \\ 0 & 1\end{array}\right)$. Then using the integral representation just obtained, we see that it suffices to prove that, given $\Phi>0$, there is a $\Phi_{0} \in \mathscr{S}(\mathrm{A})$ such that

$$
I(a) \underset{\mathrm{DEF}}{=}|a|^{s} \int_{\mathbf{A}^{\times}} \Phi\left(a t, t^{-1}\right)|t|^{2 s-1} d^{\times} t \leqslant \Phi_{0}(a)|a|^{1-A}
$$

for all $a \in \mathbf{A}^{\times}$and $s$ in the interval $1-A<(s)<A$. This is a local question. We may assume that $\Phi=\Pi \Phi_{v}$ where $\Phi_{v}$ is the characteristic function of $R_{v}^{2}$ for all finite $v$; then with obvious notations $I(a)=\Pi I_{v}\left(a_{v}\right)$. For $v$ finite the local integral $I_{v}\left(a_{v}\right)$ is zero for $\left|a_{v}\right|_{v}>1$ and is given by

$$
\begin{aligned}
I_{v}\left(a_{v}\right) & =\left|a_{v}\right|_{v}^{s} \int_{1 \leqslant|t|_{v} \leqslant\left|a_{v}\right|_{v}^{-1}|t|_{v}^{2 s-1} d^{\times} t} \\
& = \begin{cases}\frac{\left|a_{v}\right|_{v}^{1-s}-\left|a_{v}\right|_{v}^{s} q_{v}^{1-2 s}}{1-q_{v}^{1-2 s}} & \text { if } q_{v}^{2 s-1} \neq 1, \\
\left|a_{v}\right|_{v}^{s}\left(1-\frac{\log \left|a_{v}\right|_{v}}{\log q_{v}}\right) & \text { if } q_{v}^{2 s-1}=1,\end{cases}
\end{aligned}
$$

for $\left|a_{v}\right|_{v} \leqslant 1$. From this we get

$$
\begin{array}{ll}
I_{v}\left(a_{v}\right)=0 & \text { if }\left|a_{v}\right|_{v}>1, \\
I_{v}\left(a_{v}\right)=1 & \text { if }\left|a_{v}\right|_{v}=1, \\
\left|I_{v}\left(a_{v}\right)\right|<C_{v}(\varepsilon)\left|a_{v}\right|_{v}^{1-\theta-\varepsilon} & \text { if }\left|a_{v}\right|_{v}<1
\end{array}
$$


for any $\varepsilon>0$, where $\theta=\max (s, 1-s)$ and $C_{v}(\varepsilon)$ is a constant independent of $a$ which is $<1$ for almost all $v$ (for $\theta \neq \frac{1}{2}, C_{v}(\varepsilon)$ can be taken to be $q_{v}^{-\varepsilon} /\left(1-q_{v}^{1-2 \theta}\right)$ ). At a real place we have an analogous calculation: if $|\Phi(x, y)| \leqslant f(\max (x, y))$, where $f(x)$ is bounded as $x \rightarrow 0$ and rapidly decreasing as $x \rightarrow \infty$, then we find after an obvious change of variables

$$
\begin{aligned}
\left|I_{v}\left(a_{v}\right)\right|_{v} & \leqslant\left|a_{v}\right|_{v}^{s} \int_{0}^{\infty} f\left(\max \left(\left|a_{v}\right|_{v} t, t^{-1}\right)\right) t^{2 s-1} \frac{d t}{t} \\
& \leqslant 2\left|a_{v}\right|_{v}^{1-\theta} \int_{\left|a_{v}\right|_{v}^{1 / 2}}^{\infty} f(x) x^{2 \theta-2} d x
\end{aligned}
$$

which is clearly of rapid decay as $\left|a_{v}\right|_{v} \rightarrow \infty$ and is bounded by

$$
2\left|a_{v}\right|_{v}^{1-\theta-\varepsilon} \int_{0}^{\infty} f(x) x^{2 \theta+2 \varepsilon-2} d x=C_{v}(\varepsilon)\left|a_{v}\right|_{v}^{1-\theta-\varepsilon}
$$

as $\left|a_{v}\right|_{v} \rightarrow 0$ (the integral converges at $x=0$ because $2 \theta+2 \varepsilon-2>-1$ ). The calculation for complex $v$ is exactly analogous. Thus we obtain in all cases

$$
\left|I_{v}\left(a_{v}\right)\right| \leqslant C_{v}(\varepsilon) \Phi_{0 v}\left(a_{v}\right)\left|a_{v}\right|_{v}^{1-\theta-\varepsilon}
$$

with $\Phi_{0 v} \in S\left(F_{v}\right), \Phi_{0 v}=$ characteristic function of $R_{v}$ for all finite $v$, and $C_{v}(\varepsilon) \leqslant 1$ for almost all $v$, and multiplying these equations together gives the desired estimate. The uniformity in vertical strips is clear from the proof.

LEMMA 2. The integral

$$
\begin{array}{r}
V f\left(g, s ; s^{\prime}, \tau\right)=\int_{\mathbf{A}^{\times}} W f\left[\left(\begin{array}{ll}
a & 0 \\
0 & 1
\end{array}\right) g, s\right] \tau \chi_{2}^{-1}(a)|a|^{s+s^{\prime}-1} d^{\times} a \\
\\
\left(f \in H_{\chi_{1}, \chi_{2}}, g \in G_{\mathbf{A}}, s, s^{\prime} \in \mathbb{C}, \tau \in \Lambda\right)
\end{array}
$$

converges for $\mathscr{R} e\left(s^{\prime}\right)>1, \mathscr{R} e(s)>1-\frac{1}{2} \mathscr{R} e\left(s^{\prime}\right)$ and has a meromorphic continuation in $s, s^{\prime}$ given by the formula

$$
V f\left(g, s ; s^{\prime}, \tau\right)=\frac{L\left(s^{\prime}, \tau\right) L\left(2 s+s^{\prime}-1, \tau \chi_{1} \chi_{2}^{-1}\right)}{L\left(2 s, \chi_{1} \chi_{2}^{-1}\right)} Q\left(g, s ; s^{\prime}, \tau\right)
$$

where $Q\left(g, s ; s^{\prime}, \tau\right)$ for $g \in K$ is the quotient of a polynomial in $s, s^{\prime}$ and $q_{v}^{ \pm s}, q_{v}^{ \pm s^{\prime}}$ for finitely many places $v$ by a polynomial in $s$ which has no roots in the half plane $\mathscr{R} e(s)>0$. The residue of $V f$ at $s=\left(1-s^{\prime}\right) / 2\left(s^{\prime}\right.$ constant $)$ is given by

$$
\operatorname{Res}_{s=\left(1-s^{\prime}\right) / 2} V f\left(g, s ; s^{\prime}, \tau\right)= \begin{cases}-\frac{1}{2} S f\left(g, \frac{1-s^{\prime}}{2}\right) & \text { if } \tau=\chi_{1}^{-1} \chi_{2}, \\ 0 & \text { otherwise }\end{cases}
$$

Proof. The statement about the convergence is immediate by Lemma 1. By the lemma of 1.1, we have

$$
\begin{aligned}
& V f\left(g, s ; s^{\prime}, \tau\right) \\
& \quad=\sum_{i} \frac{P_{i}(s)}{L\left(2 s, \chi_{1} \chi_{2}^{-1}\right)} \int_{\mathbf{A}^{\times}} W\left[\left(\begin{array}{cc}
a & 0 \\
0 & 1
\end{array}\right) g, \Phi_{i}, \chi_{1}, \chi_{2}, s\right] \tau \chi_{2}^{-1}(a)|a|^{s+s^{\prime}-1} d^{\times} a ;
\end{aligned}
$$


we substitute for $W\left(g, \Phi, \chi_{1}, \chi_{2}, s\right)$ the formula given before Lemma 1 and observe that replacing $g$ by $\left(\begin{array}{ll}a & 0 \\ 0 & 1\end{array}\right) g$ relaces $\widetilde{g \Phi}(t, u)$ by $\widetilde{g \Phi}(a t, u)$. This gives

$$
\begin{aligned}
V f\left(g, s ; s^{\prime}, \tau\right) & =\sum_{i} \frac{P_{i}(s)}{L\left(2 s, \chi_{1} \chi_{2}^{-1}\right)} \chi_{1}(\operatorname{det} g)|\operatorname{det} g|^{s} \\
& \times \int_{\mathbf{A} \times} \int_{\mathbf{A} \times} \widetilde{g \Phi_{i}}\left(a t, t^{-1}\right) \chi_{1} \chi_{2}^{-1}(t)|t|^{2 s-1} \tau \chi_{1} \chi_{2}^{-1}(a)|a|^{2 s+s^{\prime}-1} d^{\times} t d^{\times} a .
\end{aligned}
$$

The inner integral can be rewritten by the change of variables $a \rightarrow t^{-1} a, t \rightarrow t^{-1}$ as

$$
\iint_{\left(\mathbf{A}^{\times}\right)^{2}} \widetilde{g \Phi_{i}}(a, t) \tau \chi_{1} \chi_{2}^{-1}(a)|a|^{2 s+s^{\prime}-1} \tau(t)|t|^{s^{\prime}} d^{\times} a d^{\times} t
$$

which is a Tate integral for $L\left(2 s+s^{\prime}-1, \tau \chi_{1} \chi_{2}^{-1}\right) L\left(s^{\prime}, \tau\right)$; if $g \in K$, then (since $\left.\widetilde{g \Phi_{i}} \in S_{0}\left(A^{2}\right)\right)$ it is the product of $L\left(2 s+s^{\prime}-1, \chi_{1} \chi_{2}^{-1}\right) L\left(s^{\prime}, \tau\right)$ with a polynomial in $s, s^{\prime}$ and finitely many $q_{v}^{ \pm s}, q_{v}^{ \pm s^{\prime}}$. This proves the second statement of the lemma. To compute the residue at $s=\left(1-s^{\prime}\right) / 2$, we recall that the residue of a Tate integral $L(\Phi, s, \chi)$ at $s=0, \chi=1$ is $-\Phi(0)$, so that the residue of the above double integral at $s=\left(1-s^{\prime}\right) / 2, \tau \chi_{1} \chi_{2}^{-1}=1$ is given by

$$
-\frac{1}{2} \int_{\mathbf{A} \times} \widetilde{g \Phi_{i}}(0, t) \tau(t)|t|^{s^{\prime}} d^{\times} t .
$$

But $\widetilde{g \Phi_{i}}(0, t)$ is the Fourier transform of $g \Phi_{i}(0, t)=\Phi_{i}[(0, t) g]$, so using Tate's functional equation we see that this integral is (the analytic continuation of) the Tate integral

$$
-\frac{1}{2} \int_{\mathbf{A}^{\times}} \Phi_{i}[(0, t) g] \tau^{-1}(t)|t|^{1-s^{\prime}} d^{\times} t
$$

and hence (since $\tau^{-1}=\chi_{1} \chi_{2}^{-1}$ )

$$
\begin{aligned}
\operatorname{Res}_{s=\left(1-s^{\prime}\right) / 2} V f\left(g, s ; s^{\prime}, \tau\right) & =-\frac{1}{2} \chi_{1}(\operatorname{det} g)|\operatorname{det} g|^{\left(1-s^{\prime}\right) / 2} \\
& \times \sum_{i} \frac{P_{i}\left(\left(1-s^{\prime}\right) / 2\right)}{L\left(1-s^{\prime}, \chi_{1} \chi_{2}^{-1}\right)} \int_{\mathbf{A}^{\times}} \Phi_{i}[(0, t) g] \chi_{1} \chi_{2}^{-1}(t)|t|^{1-s^{\prime}} d^{\times} t \\
= & -\frac{1}{2} \sum_{i} \frac{P_{i}\left(\left(1-s^{\prime}\right) / 2\right)}{L\left(1-s^{\prime}, \chi_{1} \chi_{2}^{-1}\right)} f\left(g, \Phi_{i}, \chi_{1}, \chi_{2}, \frac{1-s^{\prime}}{2}\right) \\
= & -\frac{1}{2} S f\left(g, \frac{1-s^{\prime}}{2}\right)
\end{aligned}
$$

as claimed.

EXERCISES. 1. Show, using the Tate functional equation, that $V f$ satisfies the functional equation

$$
V f\left(g, s ; s^{\prime}, \tau\right)=V \tilde{f}\left(g^{\prime}, 1-s ; 1-s^{\prime}, \tau^{-1}\right),
$$

where $\tilde{f} \in H\left(\chi_{2}^{-1}, \chi_{1}^{-1}\right)$ is defined by $\tilde{f}(k)=f\left(w k^{\iota}\right)$.

2. Show that $\operatorname{Res}_{s^{\prime}=1} V f\left(g, s ; s^{\prime}, 1\right)=S f(w g, s)$. 
1.3. Intertwining operators. The intertwining operator serves to compute the constant Fourier coefficient of Eisenstein series. Formally, it is the operator $M\left(\chi_{1}, \chi_{2}, s\right): H\left(\chi_{1}, \chi_{2}, s\right) \rightarrow H\left(\chi_{2}, \chi_{1}, 1-s\right)$ defined by

$$
M\left(\chi_{1}, \chi_{2}, s\right) f(g)=\int_{N_{\mathbf{A}}} f(w n g) d n .
$$

This integral converges only if $\operatorname{Res}(s)>1$, but its analytic continuation may be obtained from its effect on the elements $f\left(g, \Phi, \chi_{1}, \chi_{2}, s\right)$ :

$$
\begin{aligned}
\int_{N_{\mathbf{A}}} f\left(w n g, \Phi, \chi_{1}, \chi_{2}, s\right) d n & \\
& =\chi_{1}(\operatorname{det} g)|\operatorname{det} g|^{s} \int_{\mathbf{A}} \int_{\mathbf{A}^{\times}} \Phi[(t, t x) g] \chi_{1} \chi_{2}^{-1}(t)|t|^{2 s} d^{\times} t d x \\
& =\chi_{1}(\operatorname{det} g)|\operatorname{det} g|^{s} \int_{\mathbf{A}^{\times}} \int_{\mathbf{A}} \Phi[(t, x) g] d x \chi_{1} \chi_{2}^{-1}(t)|t|^{2 s-1} d^{\times} t
\end{aligned}
$$

which is a Tate integral for $L_{F}\left(2 s-1, \chi_{1} \chi_{2}^{-1}\right)$. Thus we see that, roughly speaking, the operator $M\left(\chi_{1}, \chi_{2}, s\right)$ has the analytic behavior of

$$
L_{F}\left(2 s-1, \chi_{1} \chi_{2}^{-1}\right) / L_{F}\left(2 s, \chi_{1} \chi_{2}^{-1}\right) .
$$

In fact, if we apply the Tate functional equation to the last expression obtained, we find that it (or rather, its analytic continuation to $\mathscr{R}_{e}(s)<\frac{1}{2}$ ) equals

$$
\chi_{1}(\operatorname{det} g)|\operatorname{det} g|^{s-1} \int_{\mathbf{A}^{\times}} \hat{\Phi}\left[(0, t) w g^{\imath}\right] \chi_{2} \chi_{1}^{-1}(t)|t|^{2-2 s} d^{\times} t \text {, }
$$

in other words we have the explicit formula

$$
M\left(\chi_{1}, \chi_{2}, s\right) f\left(g, \Phi, \chi_{1}, \chi_{2}, s\right)=f\left(w g^{\iota}, \hat{\Phi}, \chi_{1}^{-1}, \chi_{2}^{-1}, 1-s\right) .
$$

From this formula it follows in particular that $M\left(\chi_{1}, \chi_{2}, s\right)^{-1}=M\left(\chi_{2}, \chi_{1}, 1-s\right)$.

EXERCISE. Show that $\operatorname{Res}_{s^{\prime}=0} V f\left(g, s ; s^{\prime}, 1\right)=-M\left(\chi_{1}, \chi_{2}, s\right) S f(g, s)$ if $\tau=1,=0$ otherwise (compare Exercise 2 of 2.1).

As an application, we prove

LEMMA 3. Let $\phi$ be a $C^{\infty}$ function on $G_{\mathbf{A}}$ which is left and right $K$-finite, transforms by a character $\omega$ of $Z_{\mathbf{A}}$, and has compact support modulo $Z_{\mathbf{A}}$. For $s \in \mathbb{C}, \chi \in \Lambda$ define

$$
\xi_{s, \chi}(g)=\int_{N_{\mathbf{A}}} \int_{\mathbf{A}^{\times}} \varphi\left[n\left(\begin{array}{cc}
a^{-1} & 0 \\
0 & 1
\end{array}\right) g\right] \chi(a)|a|^{(s+1) / 2} d^{\times} a d n \quad\left(g \in G_{\mathbf{A}}\right)
$$

and

$$
\Xi(s, \chi)=\int_{N_{\mathbf{A}}} \xi_{s, \chi}(w n) d n
$$


Then $\Xi(s, \chi)$ is a holomorphic multiple of $L\left(s, \chi^{2} \omega^{-1}\right) / L\left(s+1, \chi^{2} \omega^{-1}\right)$ and

$$
\operatorname{Res}_{s=1} \Xi(s, \chi)= \begin{cases}c^{-1} \int_{Z_{\mathbf{A}} \backslash G_{\mathbf{A}}} \varphi(g) \bar{\chi}(\operatorname{det} g) d g & \text { if } \chi^{2}=\omega, \\ 0 & \text { otherwise, }\end{cases}
$$

where $c=\frac{1}{2} \operatorname{vol}\left(Z_{\mathbf{A}} G_{F} \backslash G_{\mathbf{A}}\right)$ as in $\S 0$.

Proof. Note that the integral defining $\xi_{s, \chi}$ converges for all $s$ (the integrand has compact support) and that $\xi_{s, \chi}$ belongs to $H\left(\chi, \chi^{-1} \omega,(s+1) / 2\right)$. Hence the integral defining $\Xi(s, \chi)=M\left(\chi, \chi^{-1} \omega,(s+1) / 2\right) \xi_{s, \chi}(1)$ converges for $\mathscr{R}_{e}(s)>1$ and can be continued analytically by the formula above: we expand

$$
\xi_{s, \chi}(g)=\sum_{\alpha \in A\left(\chi, \chi^{-1} \omega\right)}\left(\xi_{s, \chi}, f_{\alpha}\right) S f_{\alpha}\left(g, \frac{s+1}{2}\right)
$$

(the sum is finite because $\xi_{s, \chi}$ is right $K$-finite) and use the lemma of 1.1 to obtain

$$
\begin{aligned}
\Xi(s, \chi)= & \sum_{\alpha}\left(\xi_{s, \chi}, f_{\alpha}\right) \sum_{i \in I_{\alpha}} \frac{P_{\alpha, i}((s+1) / 2)}{L\left(s+1, \chi^{2} \omega^{-1}\right)} M\left(\chi, \chi^{-1} \omega, \frac{s+1}{2}\right) \\
& \times f\left(1, \Phi_{\alpha, i}, \chi, \chi^{-1} \omega, \frac{s+1}{2}\right) \\
= & L\left(s+1, \chi^{2} \omega^{-1}\right)^{-1} \sum_{\alpha, i}\left(\xi_{s, \chi}, f_{\alpha}\right) P_{\alpha, i}\left(\frac{s+1}{2}\right) \\
& \times f\left(w, \hat{\Phi}_{\alpha, i}, \chi^{-1}, \chi \omega^{-1}, \frac{1-s}{2}\right) .
\end{aligned}
$$

The analytic properties of $\Xi(s, \chi)$ are clear from this formula. In particular, it is clear that $\Xi(s, \chi)$ has no pole at $s=1$ unless $\chi \omega^{-1}=\chi^{-1}$. Using the formula

$$
\operatorname{Res}_{s=0} f\left(g, \Phi, \chi_{1}, \chi_{1}, s\right)=-\frac{1}{2} \chi_{1}(\operatorname{det} g) \Phi(0)
$$

(which is obtained by the usual Tate method) we find for $\chi^{2}=\omega$

$$
\operatorname{Res}_{s=1} \Xi(s, \chi)=\frac{1}{L(2,1)} \sum_{\alpha, i}\left(\xi_{1, \chi}, f_{\alpha}\right) P_{\alpha, i}(1) \hat{\Phi}_{\alpha, i}(0) \text {. }
$$

By the polar coordinates identity given at the end of $\S 0$ we have

$$
\hat{\Phi}(0)=\iint_{\mathbf{A}^{2}} \Phi(u, v) d u d v=c^{-1} \int_{\mathbf{A}^{\times}} \int_{K} \Phi[(0, a) k] d k|a|^{2} d^{\times} a
$$

for any $\Phi \in \mathscr{S}\left(\mathbf{A}^{2}\right)$. Hence

$$
\begin{aligned}
\sum_{i} \frac{P_{\alpha, i}(1)}{L(2,1)} \hat{\Phi}_{\alpha, i}(0) & =c^{-1} \sum_{i} \frac{P_{\alpha, i}(1)}{L(2,1)} \int_{K} f\left(k, \Phi_{\alpha, i}, \chi, \chi, 1\right) \chi^{-1}(\operatorname{det} k) d k \\
& =c^{-1} \int_{K} f_{\alpha}(k) \chi^{-1}(\operatorname{det} k) d k .
\end{aligned}
$$

Substituting this into the formula for the residue gives

$$
\operatorname{Res}_{s=1} \Xi(s, \chi)=c^{-1} \int_{K} \xi_{1, \chi}(k) \chi^{-1}(\operatorname{det} k) d k
$$


which by virtue of the Iwasawa decomposition is equivalent to the result given in the lemma.

1.4. The Selberg kernel function. Let $\varphi$ be a function with the same properties as in Lemma 3 of 1.3 and $\rho(\varphi)$ the operator

$$
\rho(\varphi) f(x)=\int_{G_{F} Z_{\mathbf{A}} \backslash G_{\mathbf{A}}} \varphi(y) f(x y) d y
$$

on the space $L^{2}=L^{2}\left(G_{F} \backslash G_{\mathbf{A}}, \omega^{-1}\right)$ of functions on $G_{F} \backslash G_{\mathbf{A}}$ which transform under $Z_{\mathbf{A}}$ by $\omega^{-1}$ and are square integrable on $G_{F} Z_{\mathbf{A}} \backslash G_{\mathbf{A}}$. This operator can clearly be represented by the kernel function

$$
K(x, y)=\sum_{\gamma \in G_{F} / Z_{F}} \varphi\left(x^{-1} \gamma y\right) .
$$

It is known (cf. [1 or 2] for an exposition) that $L^{2}$ is the direct sum of the space $L_{0}^{2}$ of cusp forms and spaces $L_{\mathrm{Eis}}^{2}$ and $L_{\mathrm{sp}}^{2}$ defined using Eisenstein series and residues of Eisenstein series, respectively, and one has a corresponding decomposition of $K$ as $K_{0}+K_{\text {Eis }}+K_{\text {sp }}$ where $K_{0}$ is the kernel function for the operator $\rho_{0}(\varphi)=$ $\rho(\varphi) \mid L_{0}^{2}$ and similarly for $K_{\text {Eis }}$ and $K_{\text {sp }}$. The theory of Eisenstein series leads to the following explicit formulas for $K_{\mathrm{Eis}}$ and $K_{\mathrm{sp}}$ :

$$
\begin{gathered}
K_{\mathrm{Eis}}(x, y)=\sum_{\chi \in \Lambda} \sum_{\alpha, \beta \in A\left(\chi, \chi^{-1} \omega\right)} \frac{1}{4 \pi i} \\
\quad \times \int_{\mathscr{R e}(u)=1 / 2}\left(\pi_{\chi, \chi^{-1} \omega, u}(\varphi) f_{\beta}, f_{\alpha}\right) E_{\alpha}(x, u) \overline{E_{\beta}(y, u)} d u, \\
K_{\mathrm{sp}}(x, y)=\frac{1}{2} \sum_{\substack{\chi \in \Lambda \\
\chi^{2}=\omega}} \bar{\chi}(\operatorname{det} x) \chi(\operatorname{det} y) \int_{G_{F} Z_{A} \backslash G_{A}} \varphi(g) \chi(\operatorname{det} g) d g
\end{gathered}
$$

here (as in 1.3) $\left\{f_{\alpha}\right\}, \alpha \in A\left(\chi, \chi^{-1} \omega\right)$ is an orthonormal basis of $H\left(\chi, \chi^{-1} \omega\right)$ consisting of $K$-finite functions and $E_{\alpha}(x, u)=E f_{\alpha}(x, u)$. The $K$-finiteness assumption on $\varphi$ implies that the triple sum on $\chi, \alpha, \beta$ in the first formula and the sum on $\chi$ in the second formula are finite (independently of $x, y$ ). The matrix coefficient $\left(\pi_{\chi, \chi^{-1} \omega}(\varphi) f_{\alpha}, f_{\beta}\right)$ occurring in the integral is the "Selberg transform" of $\varphi$. It is given explicitly by

$$
\begin{aligned}
\left(\pi_{\chi, \chi^{-1} \omega, u}(\varphi) f_{\alpha}, f_{\beta}\right)=\int_{Z_{\mathbf{A}} \backslash G_{\mathbf{A}}}\left(\pi_{\chi, \chi^{-1} \omega, u}(g) f_{\alpha}, f_{\beta}\right) \varphi(g) d g \\
\quad=\int_{Z_{\mathbf{A}} \backslash G_{\mathbf{A}}} \int_{K} S_{\alpha}(k g, u) \overline{f_{\beta}(k)} d k \varphi(g) d g \\
\quad=\int_{K} \int_{Z_{\mathbf{A}} \backslash G_{\mathbf{A}}} S_{\alpha}(g, u) \overline{f_{\beta}(k)} \varphi\left(k^{-1} g\right) d g d k \\
\quad=\int_{\mathbf{A} \times} \int_{N_{\mathbf{A}}} \int_{K} \int_{K} f_{\alpha}\left(k^{\prime}\right) \overline{f_{\beta}(k)} \varphi\left[k^{-1} n\left(\begin{array}{cc}
a & 0 \\
0 & 1
\end{array}\right) k^{\prime}\right] \chi(a)|a|^{u-1} d k d k^{\prime} d n d^{\times} a,
\end{aligned}
$$


where in the last line we have used the Iwasawa decomposition and the transformation behavior of $f_{\alpha}(g, u)$. This expresses $\left(\pi_{\chi, \chi^{-1} \omega, u}(\varphi) f_{\alpha}, f_{\beta}\right)$ as the Mellin transform of a function

$$
a \mapsto \int_{N_{\mathrm{A}}} \int_{K} \int_{K} f_{\alpha}\left(k^{\prime}\right) f_{\beta}(k) \varphi\left[k^{-1} n\left(\begin{array}{cc}
a & 0 \\
0 & 1
\end{array}\right) k^{\prime}\right] d k d k^{\prime} d n,
$$

which is of compact support on $\mathbf{A}^{\times}$, and therefore shows that it is a rapidly decreasing function of $|\operatorname{Im}(u)|$ for $\mathscr{R} e(u)$ fixed (or bounded). It follows that the integral in the formula for $K_{\text {Eis }}(x, y)$ converges normally for $x$ and $y$ in compact sets.

We will also need

Lemma. The function $K_{0}(x, y)=K(x, y)-K_{\mathrm{Eis}}(x, y)-K_{\mathrm{sp}}(x, y)$ is of rapid decay with respect to both variables.

Proof. We may assume that $\varphi$ is a triple convolution $\varphi_{1} * \varphi_{2} * \varphi_{3}$ of highly differentiable functions (since any $\varphi$ is a linear combination of such convolutions); then $K_{0, \varphi}=\varphi_{1} * K_{0, \varphi_{2}} * \varphi_{3}$ where the first convolution is with respect to $x$ and the second to $y$, and the lemma follows because the convolution of a function which is $L^{2}$ and cuspidal with a highly differentiable function is of rapid decay.

2. Calculation of $I(s)$ for $\mathscr{R}_{e}(s)>1$. We fix once and for all-two idele class characters $\omega, \tau \in \Lambda,-\mathrm{a} C^{\infty}$ function $\varphi$ on $G_{\mathbf{A}}$ which is bi- $K$-finite, transforms under $Z_{\mathbf{A}}$ by the character $\omega$, and has compact support modulo $Z_{\mathbf{A}}$, - a SchwartzBruhat function $\Phi \in \mathscr{S}_{0}\left(\mathbf{A}^{2}\right)$. Let $E\left(x, \Phi, \tau, \tau^{-1}, s\right)$ be the Eisenstein series defined in 1.1 (since $\Phi$ and $\tau$ are fixed we will often write $E(x, s)$ for this function and similarly $f(x, s)$ instead of $\left.f\left(x, \Phi, \tau, \tau^{-1}, s\right)\right)$ and $K_{0}(x, y)$ the kernel function defined in 1.4. Then both $K_{0}(x, x)$ and $E(x, s)$ are invariant under $Z_{\mathbf{A}}$ as well as left $G_{F}$-invariant (this is why we took two characters with product 1 in the definition of $E(x, s)$ ), so the integral

$$
I(s)=I(s, \varphi, \Phi, \tau)=\int_{G_{F} Z_{\mathbf{A}} \backslash G_{\mathbf{A}}} K_{0}(x, x) E(x, s) d x
$$

makes sense. The lemma of 1.4 implies that it converges for all $s$ where $E(x, s)$ is finite (i.e. all $s$, except 0 and 1 if $\tau^{2}=1$ ). It is our purpose to compute it.

2.1. Decomposition of $I(s)$. The calculation of $I(s)$ will be based on the following decomposition of the restriction of $K_{0}$ to the diagonal

$$
K_{0}(x, x)=\sum_{C} \mathscr{K}_{C}(x)+\mathscr{K}_{\infty}(x),
$$

where the summation is over all conjugacy classes $C \neq\{1\}$ in $G_{F} / Z_{F}$ and $\mathscr{K}_{C}, \mathscr{K}_{\infty}$ are defined by

$$
\begin{gathered}
\mathscr{K}_{C}(x)=\sum_{\substack{\lambda \in C^{\prime} \\
\lambda \notin P_{F} / Z_{F}}} \varphi\left(x^{-1} \lambda x\right), \\
\mathscr{K}_{\infty}(x)=\sum_{\lambda \in P_{F} / Z_{F}} \varphi\left(x^{-1} \lambda x\right)-K_{\mathrm{Eis}}(x, x)-K_{\mathrm{sp}}(x, x) .
\end{gathered}
$$


Since each of these expressions is left invariant under $P_{F}$, we obtain (for $\left.\mathscr{R} e(s)>1\right)$

$$
\begin{aligned}
I(s) & =\int_{G_{F} Z_{\mathbf{A}} \backslash G_{\mathbf{A}}} K_{0}(x, x) \sum_{\gamma \in P_{F} \backslash G_{F}} f(\gamma x, s) d x \\
& =\int_{P_{F} Z_{\mathbf{A}} \backslash G_{\mathbf{A}}} K_{0}(x, x) f(x, s) d x \\
& =\sum_{C} I_{C}(s)+I_{\infty}(s),
\end{aligned}
$$

where

$$
\begin{aligned}
I_{C}(s) & =\int_{P_{F} Z_{\mathbf{A}} \backslash G_{\mathbf{A}}} \mathscr{K}_{C}(x) f(x, s) d x, \\
I_{\infty}(s) & =\int_{P_{F} Z_{\mathbf{A}} \backslash G_{\mathbf{A}}} \mathscr{K}_{\infty}(x) f(x, s) d x .
\end{aligned}
$$

The integrals $I_{C}(s)$ and $I_{\infty}(s)$ will be calculated in 2.2 and 2.3-2.6, respectively.

Note that the above decomposition cannot be applied to the integral $\int_{G_{F} Z_{A} \backslash G_{\mathrm{A}}} K_{0}(x, x) d x$, because the individual terms $\mathscr{K}_{C}(x)$ (C not elliptic) and $\mathscr{K}_{\infty}(x)$ are not $G_{F}$-invariant. This is why our calculation of $I(s)$ and subsequent calculation of $\operatorname{tr} \rho_{0}(\varphi)$ as $\operatorname{Res}_{s=1} I(s)$ is in principle (though not in practice!) actually easier than the direct computation by integrating $K_{0}(x, x)$ over $G_{F} Z_{\mathrm{A}} \backslash G_{\mathrm{A}}$; in particular, the usual convergence problems associated with the nonelliptic conjugacy classes are avoided here and the truncation of the fundamental domain which is needed, say, in the proof of the trace formula in [2], is unnecessary. We also observe that there is no asymmetry involved in the fact that we have singled out one particular parabolic subgroup $P_{F}$ to define $\mathscr{K}_{\infty}$, because this choice is already implicit in the definition of $E(x, s)$.

We can make the above decomposition of $I(s)$ more explicit by classifying the various conjugacy classes $(\neq 1)$ as elliptic, hyperbolic, or unipotent. Each elliptic element $\lambda \in G_{F}$ generates a quadratic extension $E=F[\lambda]$ with $E^{\times} \subset G_{F}$; conversely, by fixing an embedding $E^{\times} \subset G_{F}$ for each quadratic extension $E$ of $F$ and taking $C=\{\lambda\}\left(=\right.$ conjugacy class of $\lambda$ in $\left.G_{F} / Z_{F}\right)$ where $\lambda$ runs over $E^{\times} / F^{\times}-\{1\}$, we get each elliptic conjugacy class $C$ exactly twice. Also, every hyperbolic element of $G_{F} / Z_{F}$ is conjugate to exactly two elements of the form $\left(\begin{array}{cc}\alpha & 0 \\ 0 & 1\end{array}\right)$ with $\alpha \in F^{\times}-\{1\}$, and every unipotent element is conjugate to $\left(\begin{array}{ll}1 & 1 \\ 0 & 1\end{array}\right)$. Thus we have

$$
I(s)=I_{\text {ell }}(s)+I_{\text {hyp }}(s)+I_{\text {unip }}(s)+I_{\infty}(s)
$$

with

$$
\begin{gathered}
I_{\text {ell }}(s)=\sum_{\{E: F]=2} I_{E}(s), \quad I_{E}(s)=\frac{1}{2} \sum_{\substack{\lambda \in E^{\times} / F^{\times} \\
\lambda \neq 1}} I_{\{\lambda\}}(s), \\
I_{\text {hyp }}(s)=\frac{1}{2} \sum_{\substack{\alpha \in F^{\times} \\
\alpha \neq 1}} I_{\left.\left\{\begin{array}{ll}
\alpha & 0 \\
0 & 1
\end{array}\right)\right\}}(s), \quad I_{\text {unip }}(s)=I_{\left\{\left(\begin{array}{ll}
1 & 1 \\
0 & 1
\end{array}\right)\right\}} .
\end{gathered}
$$


2.2. The contributions from conjugacy classes. Let $C$ be a nontrivial conjugacy class in $G_{F} / Z_{F}$ and $\lambda$ any element of $C$. Since every element of $C$ has the form $\gamma^{-1} \lambda \gamma$ for an element $\gamma \in G_{F}$ which is determined uniquely up to left multiplication by an element of the centralizer $G(\lambda)_{F}$ of $\lambda$ in $G_{F}$, we have

$$
\mathscr{K}_{C}(x)=\sum_{\substack{\gamma \in G(\lambda)_{F} \backslash G_{F} \\ \gamma^{-1} \lambda \gamma \notin P_{F} \backslash Z_{F}}} \varphi\left(x^{-1} \gamma^{-1} \lambda \gamma x\right) .
$$

Clearly the condition on $\gamma$ in the summation depends only on the double coset $G(\lambda)_{F} \gamma P_{F} \in G(\lambda)_{F} \backslash G_{F} / P_{F}$.

Lemma. The $\gamma$ in $G_{F}$ such that $\gamma^{-1} \lambda \gamma \notin P_{F} / Z_{F}$ form exactly one double coset $G(\lambda)_{F} \gamma_{0} P_{F}$. Moreover, $\gamma_{0}^{-1} G(\lambda)_{F} \gamma_{0} \cap P_{F}=Z_{F}$, so the elements $\gamma_{0} p\left(p \in P_{F} / Z_{F}\right)$ form a system of representatives for $G(\lambda)_{F} \backslash\left\{\gamma \in G_{F} \mid \gamma^{-1} \lambda \gamma \notin P_{F} / Z_{F}\right\}$.

Proof. If $\lambda$ is elliptic, $\lambda \in E^{\times} / F^{\times} \subset G_{F} / Z_{F}$, then the condition $\gamma^{-1} \lambda \gamma \notin P_{F} / Z_{F}$ is satisfied for all $\gamma$, so we need only show that $G(\lambda)_{F} P_{F}=G_{F}$ and $G(\lambda)_{F} \cap P_{F}=$ $Z_{F}$. But this is clear since $G_{F} / P_{F}=F \times F-\{(0,0)\} \cong E^{\times}=G(\lambda)_{F}$. If $\lambda$ is hyperbolic, then we may assume $\lambda=\left(\begin{array}{cc}\alpha & 0 \\ 0 & 1\end{array}\right)\left(\alpha \in F^{\times}, \alpha \neq 1\right)$. So $G(\lambda)=A$. Since

$$
\left(\begin{array}{ll}
a & b \\
c & d
\end{array}\right)^{-1}\left(\begin{array}{ll}
\alpha & 0 \\
0 & 1
\end{array}\right)\left(\begin{array}{ll}
a & b \\
c & d
\end{array}\right)=(a d-b c)^{-1}\left(\begin{array}{cc}
* & * \\
(1-\alpha) a c & *
\end{array}\right)
$$

the condition $\gamma^{-1} \lambda \gamma \notin P_{F} / Z_{F}$ for $\gamma=\left(\begin{array}{ll}a & b \\ c & d\end{array}\right)$ is equivalent to $a \neq 0, c \neq 0$, and it is easily seen that such a $\gamma$ has a unique representation (up to $g \rightarrow g z, p \rightarrow p z^{-1}$ with $\left.z \in Z_{F}\right)$ as $g\left(\begin{array}{ll}1 & 0 \\ 1 & 1\end{array}\right) p$ with $g \in G(\lambda)_{F}, p \in P_{F}$ (namely $g=\left(\begin{array}{cc}a & 0 \\ 0 & c\end{array}\right), p=\left(\begin{array}{cc}1 & b / a \\ 0 & d / c-b / a\end{array}\right)$ ), so the lemma is true with $\gamma_{0}=\left(\begin{array}{ll}1 & 0 \\ 1 & 1\end{array}\right)$. Similarly, if $\lambda$ is unipotent we can suppose that $\lambda=\left(\begin{array}{ll}1 & 1 \\ 0 & 1\end{array}\right)$; then $G(\lambda)=N$ and the condition $\gamma^{-1} \lambda \gamma \notin P_{F} / Z_{F}$ is equivalent to $c \neq 0$, and we can easily check that the statement of the lemma holds with $\gamma_{0}=\left(\begin{array}{ll}0 & 1 \\ 1 & 0\end{array}\right)$.

Applying the lemma to the formula preceding it we find

$$
\mathscr{K}_{C}(x)=\sum_{p \in P_{F} / Z_{F}} \varphi\left(x^{-1} p^{-1} \gamma_{0}^{-1} \lambda \gamma_{0} p x\right),
$$

from which

$$
I_{C}(s)=\int_{Z_{\mathbf{A}} \backslash G_{\mathbf{A}}} \varphi\left(x^{-1} \gamma_{0}^{-1} \lambda \gamma_{0} x\right) f(x, s) d x .
$$

But $f(x, s)=f\left(x, \Phi, \tau, \tau^{-1}, s\right)$ can be written in the form

$$
f(x, s)=\int_{Z_{A}} \Phi[(0,1) z x] \tau(\operatorname{det} z x)|\operatorname{det} z x|^{s} d z,
$$

so this can be written as an integral over all $G_{\mathbf{A}}$ :

$$
\begin{aligned}
I_{C}(s) & =\int_{G_{\mathbf{A}}} \varphi\left(x^{-1} \gamma_{0}^{-1} \lambda \gamma_{0} x\right) \Phi[(0,1) x] \tau(\operatorname{det} x)|\operatorname{det} x|^{s} d x \\
& =\int_{G_{\mathbf{A}}} \varphi\left(x^{-1} \lambda x\right) \Phi\left[(0,1) \gamma_{0}^{-1} x\right] \tau(\operatorname{det} x)|\operatorname{det} x|^{s} d x .
\end{aligned}
$$

We now look at the three types of terms individually. 
Elliptic contribution. Let $C=\{\lambda\}, \lambda \in E^{\times} / F^{\times} \subset G_{F} / Z_{F}, \gamma_{0}=1$. Since $x \rightarrow$ $\varphi\left(x^{-1} \gamma x\right)$ is left invariant under $G(\lambda)_{\mathbf{A}}=E_{\mathbf{A}}^{\times}$, we find

$$
I_{C}(s)=\int_{E_{\mathbf{A}}^{\times} \backslash G_{\mathbf{A}}} \varphi\left(x^{-1} \lambda x\right) \int_{E_{\mathbf{A}}^{\times}} \Phi[(0,1) e x] \tau(\operatorname{det} e x)|\operatorname{det} e x|^{s} d^{\times} e d x,
$$

where $d^{\times} e$ is a Haar measure on $E_{\mathbf{A}}^{\times}$and $d x$ a complementary measure on $E_{\mathbf{A}}^{\times} \backslash G_{\mathbf{A}}$. Thus, formally at least, the contribution from a given quadratic extension $E$ of $F$ is

$$
\begin{aligned}
I_{E}(s)=\int_{E_{\mathbf{A}}^{\times} \backslash G_{\mathbf{A}}}\left(\frac{1}{2} \sum_{\substack{\lambda \in E^{\times} / F^{\times} \\
\lambda \neq 1}} \varphi\left(x^{-1} \lambda x\right)\right) \\
\quad \times\left(\int_{E_{\mathbf{A}}^{\times}} \Phi[(0,1) e x] \tau(\operatorname{det} e x)|\operatorname{det} e x|^{s} d^{\times} e\right) d x .
\end{aligned}
$$

But the first expression in parentheses is easily seen to have compact support on $E_{\mathbf{A}}^{\times} \backslash G_{\mathbf{A}}$, so this expression certainly converges. As to the second factor, if we identify $E_{\mathbf{A}}^{\times}$with the ideles of $E$, det $\left.\right|_{E^{\times}: E^{\times} \rightarrow F^{\times}}$with the norm map $N_{E / F}$, $e \mapsto|\operatorname{det} e|_{F}$ with the idele norm in $E$, and $\mathscr{S}\left(\mathrm{A}^{2}\right)$ with $\mathscr{S}\left(E_{\mathrm{A}}\right)$, we see that this is just the Tate integral for $L_{E}\left(s, \tau \circ N_{E / F}\right)$.

What about the sum over $E$ ? We claim that it is in fact finite; in particular, there are no problems about the convergence of the elliptic contribution. To see this consider the function $\theta: \quad x \mapsto \operatorname{tr}(x)^{2} / \operatorname{det}(x)$ from $G_{\mathbf{A}} / Z_{\mathbf{A}}$ to $\mathbf{A}$. This map is continuous and hence maps the support of $\varphi$ to a compact set in $\mathbf{A}$. On the other hand, it is invariant under conjugation. Therefore the set of elements $\theta(\lambda)$ with $\lambda \in G(F)$ such that $\varphi\left(x^{-1} \lambda x\right) \neq 0$ for some $x \in G_{\mathbf{A}}$ is the intersection of $F$ with a compact set and hence finite. On the other hand, for $\lambda$ elliptic the field $E=F(\lambda)$ is determined by $\theta(\lambda)$, at least if $\theta(\lambda) \neq 0$ (namely $E=F[\sqrt{1-4 / \theta(\lambda)}]$ ), so we get only finitely many quadratic fields $F(\lambda)$ with $\operatorname{tr}(\lambda) \neq 0$ and $\varphi\left(x^{-1} \lambda x\right) \neq 0$ for some $x \in G_{\mathbf{A}}$. For $\operatorname{tr}(\lambda) \neq 0$ we use a similar argument: the map $\lambda \mapsto \operatorname{det}(\lambda)$ from $G_{\mathbf{A}} / Z_{\mathbf{A}}$ to the discrete set $\mathbf{A}^{\times} / \mathbf{A}^{\times 2} U$ ( $U=$ maximal compact subgroup of $\mathbf{A}^{\times}$) is continuous, so the image of $\operatorname{supp}(\varphi)$ is finite; since $F^{\times 2}$ has finite index in $F^{\times} \cap \mathrm{A}^{\times 2} U$, we deduce that there are only finitely many values of $\operatorname{det}(\lambda)\left(\bmod F^{\times 2}\right)$ with $\lambda \in G_{F}$ and $\varphi\left(x^{-1} \lambda x\right) \neq 0$ for some $x \in G_{\mathbf{A}}$, and since $F(\lambda)$ for $\lambda$ of trace zero is determined by $\operatorname{det}(\lambda)\left(\bmod F^{\times 2}\right)$ this completes the argument. (Compare the above proof with the formula for $I_{1}(s)$ given in Theorem 2, p. 323, of [12], where the fact that the sum of zeta-functions is finite when $\varphi$ has compact support follows from the inequality $\left|z^{2}+1-t^{2} / 4\right|^{2} / y^{2} \geqslant t^{2}-4$ for $t \in \mathbb{R}, z=x+i y \in H$.)

Summarizing, we have proved that $I_{\text {ell }}(s)=\sum_{[E: F]=2} I_{E}(s)$ where $I_{E}(s)$ is an entire multiple of $L_{E}\left(s, \tau \circ N_{E / F}\right)$ and $I_{E}(s)=0$ for all but finitely many $E$.

Hyperbolic contribution. This is similar; here we take

$$
\begin{gathered}
C=\{\lambda\}, \quad \lambda=\left(\begin{array}{ll}
\alpha & 0 \\
0 & 1
\end{array}\right) \quad\left(a \in F^{\times}, \alpha \neq 1\right), \\
G(\lambda)=A, \quad \gamma_{0}=\left(\begin{array}{ll}
1 & 0 \\
1 & 1
\end{array}\right), \quad(0,1) \gamma_{0}^{-1}=(1,1)
\end{gathered}
$$


and find

$$
\begin{aligned}
I_{\text {hyp }}(s)=\int_{A_{\mathbf{A}} \backslash G_{\mathbf{A}}}\left(\frac{1}{2} \sum_{\substack{\alpha \in F^{\times} \\
\alpha \neq 1}} \varphi\left[x^{-1}\left(\begin{array}{ll}
\alpha & 0 \\
0 & 1
\end{array}\right) x\right]\right) \\
\\
\times\left(\int_{A_{\mathbf{A}}} \Phi[(1,1) a x] \tau(\operatorname{det} a x)|\operatorname{det} a x|^{s} d^{\times} a\right) d x .
\end{aligned}
$$

Again the integral converges because the first factor has compact support on $A_{\mathbf{A}} \backslash G_{\mathbf{A}}$, and the inner integral

$$
\tau(\operatorname{det} x)|\operatorname{det} x|^{s} \int_{\mathbf{A}^{\times 2}} \int \Phi\left[\left(a_{1}, a_{2}\right) x\right] \tau\left(a_{1}\right) \tau\left(a_{2}\right)\left|a_{1}\right|^{s}\left|a_{2}\right|^{s} d^{\times} a_{1} d^{\times} a_{2}
$$

is a Tate integral for $L_{F}(s, \tau)^{2}$. Thus $I_{\text {hyp }}(s)$ is an entire multiple of $L_{F}(s, \tau)^{2}$.

Unipotent contribution. For $\lambda$ unipotent we must proceed differently because $x \mapsto \varphi\left(x^{-1} \lambda x\right)$ no longer has compact support modulo $G(\lambda)_{\mathbf{A}}$. Fixing $\lambda=\left(\begin{array}{ll}1 & 1 \\ 0 & 1\end{array}\right)$, $\gamma_{0}=\left(\begin{array}{ll}0 & 1 \\ 1 & 0\end{array}\right)$ and using the Iwasawa decomposition $x=\left(\begin{array}{ll}1 & 0 \\ 0 & a\end{array}\right)\left(\begin{array}{ll}t & u \\ t\end{array}\right) k$, we find

$$
\begin{aligned}
& I_{\text {unip }}(s)=\int_{K}\left(\int_{\mathbf{A}^{\times}} \varphi\left[k^{-1}\left(\begin{array}{ll}
1 & a \\
0 & 1
\end{array}\right) k\right] \tau(a)|a|^{s} d^{\times} a\right) \\
& \times\left(\int_{\mathbf{A}^{\times}} \int_{\mathbf{A}} \Phi[(t, u) k] d u|t|^{2 s-1} \tau^{2}(t) d^{\times} t\right) \tau(\operatorname{det} k) d k,
\end{aligned}
$$

and since $a \mapsto \varphi\left[k^{-1}\left(\begin{array}{cc}1 & a \\ 0 & 1\end{array}\right) k\right]$ and $t \mapsto \int_{A} \Phi[(t, u) k] d u$ are Schwartz-Bruhat functions on $\mathbf{A}$ for each $k \in K$ and $K$ is compact, we deduce that $I_{\text {unip }}(s)$ is an entire multiple of $L_{F}(s, \tau) L_{F}\left(2 s-1, \tau^{2}\right)$.

2.3. Decomposition of $I_{\infty}(s)$ and computation of $I_{\infty}^{\prime}(s)$. We now turn to $I_{\infty}(s)$, the "contribution from the cusps." We compute this by what is called Rankin's method - namely, since $f(x, s)$ is $N_{\mathbf{A}}$-invariant and transforms by $\tau(a)|a|^{s}$ under $x \mapsto a x$, we have

$$
\begin{aligned}
I_{\infty}(s) & =\int_{\mathcal{A}_{F} N_{\mathbf{A}} Z_{\mathbf{A}} \backslash G_{\mathbf{A}}} f(x, s) \mathscr{K}_{\infty, N}(x) d x \\
& =\int_{K} f(k, s) \int_{\mathbf{A}^{\times} / F^{\times}} \mathscr{K}_{\infty, N}\left[\left(\begin{array}{cc}
a & 0 \\
0 & 1
\end{array}\right) k\right] \tau(a)|a|^{s-1} d^{\times} a d k,
\end{aligned}
$$

where

$$
\mathscr{K}_{\infty, N}(x)=\int_{N_{F} \backslash N_{\mathbf{A}}} \mathscr{K}_{\infty}(n x) d n
$$

is the "constant term" of $\mathscr{K}_{\infty}$ and we have used the Iwasawa decomposition. The first term in the definition of $\mathscr{K}_{\infty}(x)$ can be written

$$
\sum_{\beta \in F} \varphi\left[x^{-1}\left(\begin{array}{ll}
1 & \beta \\
0 & 1
\end{array}\right) x\right]+\sum_{\substack{\alpha \in F^{\times} \\
\alpha \neq 1}} \sum_{\beta \in F} \varphi\left[x^{-1}\left(\begin{array}{cc}
1 & \beta \\
0 & 1
\end{array}\right)^{-1}\left(\begin{array}{cc}
\alpha & 0 \\
0 & 1
\end{array}\right)\left(\begin{array}{ll}
1 & \beta \\
0 & 1
\end{array}\right) x\right],
$$

and the constant term of this is

$$
\sum_{\beta \in F} \varphi\left[x^{-1}\left(\begin{array}{cc}
1 & \beta \\
0 & 1
\end{array}\right) x\right]+\sum_{\alpha \in F^{\times}-\{1\}} \int_{\mathbf{A}} \varphi\left[x^{-1}\left(\begin{array}{cc}
\alpha & u \\
0 & 1
\end{array}\right) x\right] d u .
$$


Write the other two terms in $\mathscr{K}_{\infty}(x)$ as $-h(x, x)$, where $h(x, y)=-K_{\text {Eis }}(x, y)-$ $K_{\text {sp }}(x, y)$. Since $h(x, y)$ is (left) $N_{F}$-invariant with respect to each variable, the constant term of $h(x, x)$ can be expressed as a diagonal sum of Fourier coefficients of $h(x, y)$ with respect to $x$ and $y$ separately by the identity

$$
\begin{aligned}
\int_{N_{F} \backslash N_{\mathbf{A}}} h(n x, n x) d n & =\iint_{\left(N_{F} \backslash N_{\mathbf{A}}\right)^{2}} h\left(n_{1} x, n_{2} x\right) n_{1} d n_{2} \\
& +\sum_{\alpha \in F^{\times}} \iint_{(\mathbf{A} / F)^{2}} h\left[\left(\begin{array}{cc}
1 & u_{1} \\
0 & 1
\end{array}\right) x,\left(\begin{array}{cc}
1 & u_{2} \\
0 & 1
\end{array}\right) x\right] \psi\left(\alpha u_{1}-\alpha u_{2}\right) d u_{1} d u_{2} .
\end{aligned}
$$

In the first term on the right we can replace $h(x, y)=-K(x, y)+K_{0}(x, y)$ by $-K(x, y)$, since $K_{0}$ by definition has no constant term with respect to $x$ or $y$; in the second term we can replace $h$ by $-K_{\text {Eis }}$ since $K_{\text {sp }}(x, y)$ has no nonconstant Fourier coefficients. We also use the identity

$$
K(x, y)=\sum_{\substack{\nu_{1}, \nu_{2} \in N_{F} \\
\alpha \in F^{\times}}} \varphi\left[x^{-1} \nu_{1}^{-1}\left(\begin{array}{cc}
\alpha & 0 \\
0 & 1
\end{array}\right) w \nu_{2} y\right]+\sum_{\substack{\nu \in N_{F} \\
\alpha \in F^{\times}}} \varphi\left[x^{-1}\left(\begin{array}{cc}
\alpha & 0 \\
0 & 1
\end{array}\right) \nu y\right]
$$

(Bruhat decomposition) to rewrite the first term as

$$
-\iint_{N_{\mathbf{A}}^{2}} \sum_{\alpha \in F^{\times}} \varphi\left[x^{-1} n_{1}^{-1}\left(\begin{array}{cc}
\alpha & 0 \\
0 & 1
\end{array}\right) w n_{2} x\right] d n_{1} d n_{2}-\sum_{\alpha \in F^{\times}} \int_{\mathbf{A}} \varphi\left[x^{-1}\left(\begin{array}{cc}
\alpha & u \\
0 & 1
\end{array}\right) x\right] d u .
$$

Recombining the terms and making the substitution $u_{i} \rightarrow \alpha u_{i}$ in the integral involving $K_{\text {Eis }}$, we find that we have obtained the decomposition

$$
\mathscr{K}_{\infty, N}(x)=\mathscr{K}_{\infty}^{\prime}(x)+\mathscr{K}_{\infty}^{\prime \prime}(x)+\mathscr{K}_{\infty}^{\prime \prime \prime}(x)
$$

with

$$
\begin{aligned}
& \mathscr{K}_{\infty}^{\prime}(x)=\sum_{\beta \in F} \varphi\left[x^{-1}\left(\begin{array}{cc}
1 & \beta \\
0 & 1
\end{array}\right) x\right]-\int_{\mathbf{A}} \varphi\left[x^{-1}\left(\begin{array}{ll}
1 & u \\
0 & 1
\end{array}\right) x\right] d u, \\
& \mathscr{K}_{\infty}^{\prime \prime}(x)=-\iint_{N_{\AA}^{2}} \sum_{\alpha \in F^{\times}} \varphi\left[x^{-1} n_{1}^{-1}\left(\begin{array}{cc}
\alpha & 0 \\
0 & 1
\end{array}\right) w n_{2} x\right] d n_{1} d n_{2}, \\
& \mathscr{K}_{\infty}^{\prime \prime \prime}(x)=-\sum_{\alpha \in F^{\times}} \iint_{\left(N_{\mathbf{A}} / N_{F}\right)^{2}} K_{\mathrm{Eis}}\left[n_{1}\left(\begin{array}{cc}
\alpha & 0 \\
0 & 1
\end{array}\right) x, n_{2}\left(\begin{array}{cc}
\alpha & 0 \\
0 & 1
\end{array}\right) x\right] \psi\left(n_{1} n_{2}^{-1}\right) d n_{1} d n_{2} .
\end{aligned}
$$

We write the corresponding decomposition of $I_{\infty}$ as

$$
\begin{aligned}
I_{\infty}(s) & =I_{\infty}^{\prime}(s)+I_{\infty}^{\prime \prime}(s)+I_{\infty}^{\prime \prime \prime}(s), \\
I_{\infty}^{(i)}(s) & =\int_{A_{F} N_{\mathbf{A}} Z_{\mathbf{A}} \backslash G_{\mathbf{A}}} f(x, s) \mathscr{K}_{\infty}^{(i)}(x) d x \\
& =\int_{K} f(k, s) \int_{\mathbf{A}^{\times} / F^{\times}} \mathscr{K}_{\infty}^{(i)}\left[\left(\begin{array}{ll}
a & 0 \\
0 & 1
\end{array}\right) k\right] \tau(a)|a|^{s-1} d^{\times} a d k .
\end{aligned}
$$

The integrals $I_{\infty}^{\prime \prime}(s)$ and $I_{\infty}^{\prime \prime \prime}(s)$ are fairly hard to compute and will be treated in the next two sections, but the integral $I_{\infty}^{\prime}(s)$ is easy and will be done now. By the 
Poisson summation formula,

$$
\begin{aligned}
& \mathscr{K}_{\propto}^{\prime}(x)=\sum_{\alpha \in F^{\times}} \int_{\mathbf{A}} \varphi\left[x^{-1}\left(\begin{array}{ll}
1 & u \\
0 & 1
\end{array}\right) x\right] \psi(\alpha u) d u \\
& =\sum_{\alpha \in F^{\times}} \int_{\mathbf{A}} \varphi\left[x^{-1}\left(\begin{array}{cc}
\alpha & 0 \\
0 & 1
\end{array}\right)^{-1}\left(\begin{array}{cc}
1 & u \\
0 & 1
\end{array}\right)\left(\begin{array}{ll}
\alpha & 0 \\
0 & 1
\end{array}\right) x\right] \psi(u) d u,
\end{aligned}
$$

so

$$
\begin{aligned}
I_{\infty}^{\prime}(s) & =\int_{K} f(k, s) \int_{\mathbf{A}^{\times}} \int_{\mathbf{A}} \varphi\left[k^{-1}\left(\begin{array}{ll}
a & 0 \\
0 & 1
\end{array}\right)^{-1}\left(\begin{array}{cc}
1 & u \\
0 & 1
\end{array}\right)\left(\begin{array}{ll}
a & 0 \\
0 & 1
\end{array}\right) k\right] \\
& \times \psi(u) d u \tau(a)|a|^{s-1} d^{\times} a d k \\
= & \int_{K} f(k, s) \int_{\mathbf{A}^{\times}} \int_{\mathbf{A}} \varphi\left[k^{-1}\left(\begin{array}{cc}
1 & u \\
0 & 1
\end{array}\right) k\right] \psi(a u) d u \tau(a)|a|^{s} d^{\times} a d k,
\end{aligned}
$$

where in the last line we have made the substitution $u \rightarrow a u$. The function

$$
u \mapsto \varphi\left[k^{-1}\left(\begin{array}{cc}
1 & u \\
0 & 1
\end{array}\right) k\right]
$$

and hence also its Fourier transform

$$
a \mapsto \int_{\mathbf{A}} \varphi\left[k^{-1}\left(\begin{array}{cc}
1 & u \\
0 & 1
\end{array}\right) k\right] \psi(a u) d u,
$$

are Schwartz-Bruhat functions for any $k$, so the inner double integral is a Tate integral for $L_{F}(s, \tau)$; using the compactness of $K$ and the $K$-finiteness of $\varphi$, we deduce that $I_{\infty}^{\prime}(s)$ is an entire multiple of $L_{F}(s, \tau) L_{F}\left(2 s, \tau^{2}\right)$.

2.4. Computation of $I_{\infty}^{\prime \prime}(s)$. Substituting the identity

$$
\begin{gathered}
\varphi\left[k^{-1}\left(\begin{array}{ll}
a^{-1} & 0 \\
0 & 1
\end{array}\right)\left(\begin{array}{cc}
1 & u_{1} \\
0 & 1
\end{array}\right)\left(\begin{array}{cc}
\alpha & 0 \\
0 & 1
\end{array}\right) w\left(\begin{array}{cc}
1 & u_{2} \\
0 & 1
\end{array}\right)\left(\begin{array}{ll}
a & 0 \\
0 & 1
\end{array}\right) k\right] \\
=\omega(a) \varphi\left[k^{-1}\left(\begin{array}{cc}
1 & a^{-1} u_{1} \\
0 & 1
\end{array}\right)\left(\begin{array}{cc}
a^{-2} \alpha & 0 \\
0 & 1
\end{array}\right) w\left(\begin{array}{cc}
1 & a^{-1} u_{2} \\
0 & 1
\end{array}\right) k\right]
\end{gathered}
$$

into the formula for $I_{\infty}^{\prime \prime}(s)$ and making the change of variables $u_{1} \rightarrow a u_{1}, u_{2} \rightarrow a u_{2}$, we find-after an interchange of summation and integration which will be justified below - the formula

$$
I_{\infty}^{\prime \prime}(s)=-\int_{N_{\mathbf{A}}} \int_{\mathbf{A}^{\times} / F^{\times}} \sum_{\alpha \in F^{\times}} \xi_{s}\left(w n, a^{2} \alpha\right) \tau \omega(a) d^{\times} a d n,
$$

where we have set

$$
\begin{aligned}
\xi_{s}(g, a)=|a|^{(s+1) / 2} \int_{K} f(k, s) \int_{N_{\mathbf{A}}} \varphi\left[k^{-1} n\left(\begin{array}{ll}
a^{-1} & 0 \\
0 & 1
\end{array}\right) g k\right] d n d k & \left(s \in \mathbb{C}, a \in \mathbf{A}^{\times}, g \in G_{\mathbf{A}}\right) .
\end{aligned}
$$

We apply to it the following lemma.

LemmA. Let $\xi$ be a smooth function of compact support on $\mathbb{A}^{\times}$and $\omega \in \Lambda$. Then

$$
\int_{\mathbf{A} \times / F^{\times}} \sum_{\alpha \in F^{\times}} \xi\left(\alpha a^{2}\right) \omega(a) d^{\times} a=\frac{1}{2} \sum_{\chi^{2}=\omega} \xi_{\chi}
$$


where the summation is over all $\chi \in \Lambda$ with $\chi^{2}=\omega$ and

$$
\xi_{\chi}=\int_{\mathbf{A}^{\times}} \xi(a) \chi(a) d^{\times} a .
$$

Proof. We first observe that, since the function $a \mapsto \sum_{\alpha \in F^{\times}} \xi(\alpha a)$ has compact support on $\mathrm{A}^{\times} / F^{\times}$and the map $a \mapsto a^{2}$ from $\mathrm{A}^{\times} / F^{\times}$to itself is proper, the integrand on the left-hand side of the equation has compact support, so the integral converges. We now use the decomposition $\mathbf{A}^{\times}=\mathbb{R}_{+}^{\times} \times \mathbf{A}_{1}^{\times}$(see §0) and set

$$
\xi_{1}\left(a_{1}\right)=\int_{\mathbf{R}_{+}^{\times}} \xi\left(a_{1} t\right) d^{\times} t \quad\left(a_{1} \in \mathbf{A}_{1}^{\times}\right) ;
$$

then

$$
\xi_{\chi}=\int_{\mathbf{A}_{1}^{\times}} \xi_{1}\left(a_{1}\right) \chi\left(a_{1}\right) d^{\times} a_{1}
$$

is just the $\bar{\chi}$ th Fourier coefficient of $\xi_{1}$ and we find

$$
\begin{aligned}
\int_{\mathbf{A}^{\times} / F^{\times}} \omega(a) \sum_{\alpha \in F^{\times}} \xi\left(\alpha a^{2}\right) d^{\times} a & =\int_{\mathbf{A}_{1}^{\times} / F^{\times}} \omega\left(a_{1}\right) \sum_{\alpha \in F^{\times}} \int_{\mathbf{R}_{x}^{+}} \xi\left(\alpha a_{1}^{2} t^{2}\right) d^{\times} t d^{\times} a_{1} \\
& =\int_{\mathbf{A}_{1}^{\times} / F} \omega\left(a_{1}\right) \sum_{\alpha \in F^{\times}} \frac{1}{2} \xi_{1}\left(\alpha a_{1}^{2}\right) d^{\times} a_{1} \\
& =\int_{\mathbf{A}_{1}^{\times} / F^{\times}} \omega\left(a_{1}\right) \frac{1}{2} \sum_{\chi \in \Lambda} \xi_{\chi} \bar{\chi}\left(a_{1}^{2}\right) d^{\times} a_{1}
\end{aligned}
$$

(Poisson summation formula),

and since the integral of $\bar{\chi}\left(a_{1}^{2}\right) \omega\left(a_{1}\right)$ over $\mathbf{A}_{1}^{x} / F^{\times}$is 1 for $\chi^{2}=\omega$ and 0 otherwise this completes the proof.

We apply the lemma to $\xi_{s}(g, \cdot)$, which has compact support for $g$ fixed. The function

$$
g \mapsto \int_{K} \varphi\left(k^{-1} g k\right) f(k, \Phi, s) d k
$$

is $K$-finite on both sides (because the restriction of $f$ to $K$ is), and it follows that the function $a \mapsto \xi_{s}(g, a)$ transforms by a finite-dimensional representation of $U$ (the maximal compact subgroup of $\mathbf{A}^{\times}$) which is independent of $g$. Therefore the Fourier coefficient $\xi_{s, \chi}(g)$, defined in the lemma is zero for all $\chi$ not belonging to a certain finite subset of $\Lambda$ which depends only on $\varphi$. The formula

$$
I_{\infty}^{\prime \prime}(s)=-\frac{1}{2} \sum_{\chi^{2}=\omega \tau} \int_{N_{\mathrm{A}}} \xi_{s, \chi}(w n) d n
$$

which we have obtained therefore expresses $I_{\infty}^{\prime \prime}(s)$ as a finite sum of integrals. Moreover, the function $\xi_{s, \chi}$ belongs to $H\left(\chi, \chi^{-1} \omega,(s+1) / 2\right)$ and so these integrals are precisely the intertwining operators discussed in 1.3 and known to converge absolutely. Thus the final expression obtained is finite, and since the whole argument could have been applied with $s$ replaced by $\mathscr{R}_{e}(s)$ and $\varphi$ by a positive and 
bi- $K$-invariant majorant, we see a posteriori that our integrals were absolutely convergent at every stage and all the steps of our computation valid. The calculation given at the end of 1.3 (together with the usual argument about the $K$-finiteness of $\varphi)$ now shows that $I_{\infty}^{\prime \prime}(s)$ is an entire multiple of $L(s, \tau) L\left(2 s, \tau^{2}\right) / L(s+1, \tau)$.

2.5. Computation of $I_{\infty}^{\prime \prime \prime}(s)$. We need a closed expression and an estimate for

$$
\int_{\left(N_{\mathbf{A}} / N_{F}\right)^{2}} K_{\mathrm{Eis}}\left(n_{1} x, n_{2} x\right) \psi\left(n_{1}\right) \psi\left(n_{2}^{-1}\right) d n_{1} d n_{2}
$$

To obtain them, replace $K_{\text {Eis }}$ by its expression as a sum of integrals, recalling from 1.3 that the sum is finite and the integrals normally convergent on compact sets. It follows that the above integral equals

$$
\sum_{\chi} \sum_{\alpha, \beta} \frac{1}{4 \pi i} \int_{\mathscr{R} e(u)=1 / 2}\left(\pi_{\chi, \chi^{-1} \omega, u}(\varphi) f_{\beta}, f_{\alpha}\right) W_{\alpha}(x, u) \overline{W_{\beta}(x, u)} d u
$$

where $W_{\alpha}=W f_{\alpha}$ is the Whittaker function defined in 1.2. We have to check that this can be integrated against $f(x, s)$ for $\mathscr{R} e(s)$ sufficiently large. Using Lemma 1 of 1.2 , we can majorize the resulting double integral by

$$
\int_{\mathbf{A} \times} \int_{-\infty}^{\infty}\left|\left(\pi_{\chi, \chi^{-1} \omega, 1 / 2+i r}(\varphi) f_{\alpha}, f_{\beta}\right)\right| P(r) \Phi_{1}(a) \Phi_{2}(a)|a|^{\operatorname{Re}_{e}(s)-\varepsilon} d r d^{\times} a,
$$

where $P(r)$ is an "elementary function", $\Phi_{1}$ and $\Phi_{2}$ are Schwartz-Bruhat functions, and $\varepsilon$ is an arbitrary positive number. Since this clearly converges for $\mathscr{R} e(s)>1+\varepsilon$, we see that the integral $I_{\infty}^{\prime \prime \prime}(s)$ converges absolutely for $\mathscr{R} e(s)>1$ and is given in that domain by the formula

$$
I_{\infty}^{\prime \prime \prime}(s)=-\sum_{\chi} \sum_{\alpha, \beta \in A\left(\chi, \chi^{-1} \omega\right)} \frac{1}{4 \pi i} \int_{\mathscr{R}_{e}(u)=1 / 2}\left(\pi_{\chi, \chi^{-1} \omega, u}(\varphi) f_{\beta}, f_{\alpha}\right) J_{\alpha, \beta}(s, u) d u
$$

where

$$
J_{\alpha, \beta}(s, u)=\int_{N_{\mathbf{A}} Z_{\mathbf{A}} \backslash G_{\mathbf{A}}} f(x, s) W_{\alpha}(x, u) \overline{W_{\beta}(x, 1-\bar{u})} d x
$$

(we have replaced $u$ by $1-\bar{u}$ in $W_{\beta}$ to make $J_{\alpha \beta}$ holomorphic in $u$ ), which can also be written

$$
J_{\alpha, \beta}(s, u)=\int_{N_{\mathbf{A}} \backslash G_{\mathbf{A}}} \Phi[(0,1) x] W_{\alpha}(x, u) \overline{W_{\beta}(x, 1-\bar{u})} \tau(\operatorname{det} x)|\operatorname{det} x|^{s} d x
$$

We need the analytic properties of $J_{\alpha \beta}(s, u)$. They are given by the following four propositions.

Proposition 1. Let $f_{1}, f_{2} \in H\left(\chi_{1}, \chi_{2}\right)$ be K-finite, $\tau \in \Lambda, \Phi \in S\left(A^{2}\right), u \in \mathbf{C}$. Let $J(s, u)=J\left(f_{1}, f_{2} ; \Phi ; \tau ; s, u\right)$ be the function defined for $\mathscr{R} e(s)$ sufficiently large 
by

$$
J(s, u)=\int_{N_{\mathbf{A}} \backslash G_{\mathbf{A}}} \Phi[(0,1) x] W f_{1}(x, u) \overline{W f_{2}(x, 1-\bar{u})} \tau(\operatorname{det} x)|\operatorname{det} x|^{s} d x .
$$

Then $J(s, u)$ is given by an expression of the form

$$
J(s, u)=\frac{R(s, u)}{P(u)} \frac{L(s, \tau)^{2} L\left(s+1-2 u, \chi_{1}^{-1} \chi_{2} \tau\right) L\left(s+2 u-1, \chi_{1} \chi_{2}^{-1} \tau\right)}{L\left(2-2 u, \chi_{1}^{-1} \chi_{2}\right) L\left(2 u, \chi_{1} \chi_{2}^{-1}\right)}
$$

where $P(u)$ is a polynomial in $u$ and finitely many $q_{v}^{u}, q_{v}^{-u}$ which has no zeros for $0<\mathscr{R e}(u)<1$ and $R(s, u)$ is a polynomial in $s, u$ and $q_{v}^{s}, q_{v}^{-s}, q_{v}^{u}, q_{v}^{-u}$ for finitely many $v$.

Proposition 2. The residue of $J(s, u)$ at $u=(1-s) / 2(s$ fixed $), \tau=\chi_{1}^{-1} \chi_{2}$, is given by

$$
\operatorname{Res}_{u=(1-s) / 2} J(s, u)=-\frac{1}{2} \int_{A_{\mathbf{A}} \backslash G_{\mathbf{A}}} S f_{1}\left(x, \frac{1-s}{2}\right) \overline{S f_{2}\left(w x, \frac{1+\bar{s}}{2}\right)} f(x, s) d x .
$$

Proposition 3. The function $J$ satisfies the functional equation

$$
J\left(f_{1}, f_{2} ; \Phi, \tau ; s, u\right)=J\left(\tilde{f}_{1}, \tilde{f}_{2} ; \hat{\boldsymbol{\Phi}}, \tau^{-1} ; 1-s, u\right)
$$

where $\tilde{f}_{i} \in H\left(\chi_{2}^{-1}, \chi_{1}^{-1}\right)$ is defined by $\tilde{f}_{i}(k)=f_{i}\left(w k^{\iota}\right)(k \in K)$.

Combining Propositions 2 and 3, we obtain

$$
\underset{u=s / 2}{\operatorname{Res}} J(s, u)=-\frac{1}{2} \int_{A_{\mathbf{A}} \backslash G_{\mathbf{A}}} S \tilde{f}_{1}\left(x, \frac{s}{2}\right) \overline{S \tilde{f}_{2}(w x, 1-\bar{s} / 2)} f^{\prime}(x, s) d x,
$$

for $\tau=\chi_{1} \chi_{2}^{-1}$ (the residue is 0 for other $\tau$ ), where

$$
\begin{aligned}
f^{\prime}(x, s) & =f\left(x, \hat{\Phi}, \tau^{-1}, \tau, 1-s\right) \\
& =\bar{\tau}(\operatorname{det} x)|\operatorname{det} x|^{1-s} \int_{\mathbf{A}^{\times}} \hat{\Phi}[(0, t) x] \bar{\tau}^{2}(t)|t|^{2-2 s} d^{\times} t .
\end{aligned}
$$

Proposition 4. $\lim _{s \rightarrow 1}(s-1)^{2} J(s, u)=\delta_{\tau, 1} \cdot C \cdot\left(f_{1}, f_{2}\right)$ (independent of $u$ ), where $C=\iint_{\mathbf{A}^{2}} \Phi(x, y) d x d y$.

Proof of Proposition 1. By the lemma in 1.1 , we may assume that

$$
S f_{2}(x, u)=\frac{P(u)}{L\left(2 u, \chi_{1} \chi_{2}^{-1}\right)} f\left(x, \Phi_{2}, \chi_{1}, \chi_{2}, u\right),
$$

where $\Phi_{2}$ is a Schwartz-Bruhat function and $P$ is a rational function of $u$ and finitely many $q_{v}^{u}$; then $E f_{2}(x, u)$ and $W f_{2}(x, u)$ are $P(u) / L\left(2 u, \chi_{1} \chi_{2}^{-1}\right)$ times the functions $E\left(x, \Phi_{2}, \chi_{1}, \chi_{2}, u\right)$ and $W\left(\chi, \Phi_{2}, \chi_{1}, \chi_{2}, u\right)$ whose analytic properties were given in 1.1 and 1.2. It follows from the estimate of Whittaker functions given in 1.2 that the integral $J(s, u)$ converges absolutely for $\mathscr{R}_{e}(s)>2 \max \left(\mathscr{R} e(u), \mathscr{R}_{e}(1-u)\right)$ (the shaded region in Figure 1), and although we were originally interested in $(s, u)$ with $\mathscr{R e}(u)=\frac{1}{2}$ and $\mathscr{R e}(s)>1$ we now pass by analytic continuation to a region 
where $\mathscr{R} e(u)<0$. Then $\mathscr{R} e(1-\bar{u})>1$ and we can replace $W f_{2}(x, 1-\bar{u})$ by its original definition as an integral over $f$, obtaining

$$
\begin{aligned}
J(s, u)=\beta(u) \int_{G_{\mathbf{A}}} \Phi[(0,1) x] W f_{1}( & x, u) \\
& \times \overline{f\left(w x, \Phi_{2}, \chi_{1}, \chi_{2}, 1-\bar{u}\right)} \tau(\operatorname{det} x)|\operatorname{det} x|^{s} d x,
\end{aligned}
$$

where

$$
\beta(u)=\overline{P(1-\bar{u})} / L\left(2-2 u, \chi_{1}^{-1} \chi_{2}\right)
$$

(the $\psi(n)$ in the definition of $W f\left(x, \Phi_{2}, \chi_{1}, \chi_{2}, 1-\bar{u}\right)$ is absorbed by the function $\Phi[(0,1) x] W f_{1}(x, u)$ which transforms by $\psi$ under $\left.N_{\mathbf{A}}\right)$. We now substitute for $f\left(x, \Phi_{2}, \chi_{1}, \chi_{2}, 1-\bar{u}\right)$ its definition as an integral over $\mathbf{A}^{\times}$(convergent for $\mathscr{R}_{e}(u)<$ 0 ) and interchange the order of integration to obtain

$$
\begin{aligned}
J(s, u)=\beta(u) \int_{\mathbf{A} \times} \int_{G_{\mathbf{A}}} & \Phi[(0,1) x] \overline{\Phi_{2}[(t, 0) x]} \\
& \times W f_{1}(x, u) \tau \chi_{1}^{-1}(\operatorname{det} x)|\operatorname{det} x|^{s+1-u} d k|t|^{2-2 u} \chi_{1}^{-1} \chi_{2}(t) d^{\times} t ;
\end{aligned}
$$

in this integral we replace $t$ by $t^{-1}$ and then $x$ by $\left(\begin{array}{ll}t & 0 \\ 0 & 1\end{array}\right) x$ in the inner integral and interchange the order of integration again to obtain

$$
J(s, u)=\beta(u) \int_{G_{\mathbf{A}}} \Psi(x) V f_{1}(x, u ; s, \tau) \tau \chi_{1}^{-1}(\operatorname{det} x)|\operatorname{det} x|^{s+1-u} d x
$$

where $\Psi(x)=\Phi[(0,1) x] \bar{\Phi}_{2}[(1,0) x]$ and $V f_{1}(x, u ; s, \tau)$ is the function defined in Lemma 2 of 1.2 (this transformation is due to Shalika). Since the proof that the original integral converged used an estimate only for the absolute value of $W f_{1}$ and since all other steps could have been carried out for $\tau, \chi_{1}$ and $\chi_{2}$ trivial, $s$ and $u$ real, and $\Phi$ and $\Phi_{2}$ positive, we see that the double integral over $G_{\mathbf{A}} \times \mathbf{A} \times$ is absolutely convergent and the interchanges carried out therefore justified.

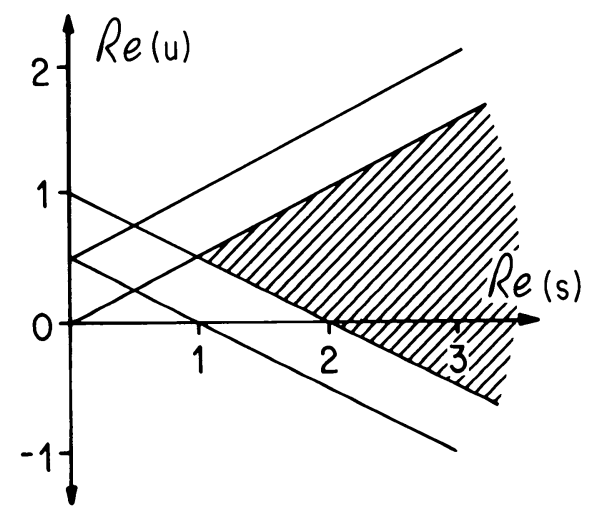

FIGURE 1 
Now the analytic properties of $V f_{1}(x, u ; s, \tau)$ as a function of $s$ and $u$ were given in 1.2 , but since we do not know the behavior of this function with respect to the adelic variable $x$ we cannot immediately make use of them in the above integral. However, $V f_{1}(x, u ; s, \tau)$ depends only on the section $S f_{1}(x, u) \in H\left(\chi_{1}, \chi_{2}, u\right)$, and writing $f_{1}$ in terms of the standard orthonormal basis $\left\{f_{\gamma}\right\}_{\gamma \in A\left(\chi_{1}, \chi_{2}\right)}$ of $H\left(\chi_{1}, \chi_{2}\right)$ we see that

$$
\begin{aligned}
S f_{1}(x, u) & =\pi_{\chi_{1}, \chi_{2}, u}(x) f_{1}(1) \\
& =\sum_{\gamma \in A\left(\chi_{1}, \chi_{2}\right)}\left(\pi_{\chi_{1}, \chi_{2}, u}(x) f_{1}, f_{\gamma}\right) f_{\gamma}(1)
\end{aligned}
$$

(here $\pi_{\chi_{1}, \chi_{2}, u}$ is the representation of $G_{\mathbf{A}}$ on $H\left(\chi_{1}, \chi_{2}, u\right)$, transferred to $H\left(\chi_{1}, \chi_{2}\right)$ by means of $S$ ) and hence

$$
V f_{1}(x, u ; s, \tau)=\sum_{\gamma}\left(\pi_{\chi_{1}, \chi_{2}, u}(x) f_{1}, f_{\gamma}\right) V_{\gamma}(1, u ; s, \tau) .
$$

The advantage of this formula for $V$ is that the dependence on $x$ and that on $u$ have been separated. Substituting it into the above expression for $J$ and interchanging the summation and integration, we would obtain

$$
\begin{aligned}
J(s, u)=\beta(u) \sum_{\gamma} V_{\gamma}(1, u ; s, \tau) \int_{G_{A}} \Psi(x)\left(\pi_{\chi_{1}, \chi_{2}, u}(x) f_{1}, f_{\gamma}\right) & \\
& \times \tau \chi_{1}^{-1}(\operatorname{det} x)|\operatorname{det} x|^{s+1-u} d x .
\end{aligned}
$$

We now show that this interchange of summation and integration is justified and that the resulting sum of integrals is finite. To do this, we use the standard device of introducing an elementary idempotent $\xi$ on $K$ (= finite sum of characters of irreducible representations divided by the dimension of the representation) such that

$$
\int_{K} \Psi(k x) \xi(k) d k=\Psi(x) .
$$

This is possible because $\Psi$ is the restriction to $G_{\mathrm{A}}$ of a function in $\mathscr{S}_{0}\left(\mathrm{~A}^{4}\right)$ and hence $K$-finite. We substitute this into the absolutely convergent double integral for $J(s, u)$ which was obtained above, getting

$$
\begin{aligned}
& J(s, u)=\beta(u) \int_{G_{\mathbf{A}}} \int_{K} \int_{\mathbf{A}^{\times}} W f_{1}\left[\left(\begin{array}{cc}
a & 0 \\
0 & 1
\end{array}\right) x, u\right] \tau \bar{\chi}_{2}(a)|a|^{s+u-1} \\
& \times \Psi(k x) \xi(k) \tau \bar{\chi}_{1}(\operatorname{det} x)|\operatorname{det} x|^{s+1} d^{\times} a d k d x .
\end{aligned}
$$

Because the functions $x \mapsto \Psi(k x)(k \in K)$ are bounded by a fixed Schwartz-Bruhat function, this triple integral converges absolutely (in the region $0>\operatorname{Re}(u)>1$ $-\frac{1}{2} \mathscr{R e}(s)$ ), so we interchange the integrations over $G_{\mathbf{A}}$ and $K$, make the change of variables $x \rightarrow k^{-1} x$ and interchange back to get

$$
\begin{array}{rl}
J(s, u)=\beta(u) \int_{G_{\mathbf{A}}} \int_{\mathbf{A}^{\times}} \int_{K} & W f_{1}\left[\left(\begin{array}{ll}
a & 0 \\
0 & 1
\end{array}\right) k x\right] \xi\left(k^{-1}\right) \tau \bar{\chi}_{1}(\operatorname{det} k) d k \\
& \times \tau \bar{\chi}_{2}(a)|a|^{s+1-u} d^{\times} a \Psi(x) \tau \bar{\chi}_{1}(\operatorname{det} x)|\operatorname{det} x|^{s+1-u} d x .
\end{array}
$$


Now, observing that the function $k \mapsto \xi\left(k^{-1}\right) \tau \bar{\chi}_{1}(\operatorname{det} k)$ is an elementary idempotent on $K$ and that the expansion

$$
S f_{1}(y x, u)=\sum_{\gamma}\left(\pi_{\chi_{1}, \chi_{2}, u}(x) f_{1}, f_{\gamma}\right) S_{\gamma}(y, u)
$$

converges normally on compact sets, we find that

$$
\begin{aligned}
\int_{K} S f_{1}(y k x, u) \xi\left(k^{-1}\right) & \tau \bar{\chi}_{1}(\operatorname{det} k) d k \\
& =\sum_{\gamma} \int_{K}\left(\pi_{\chi_{1}, \chi_{2}, u}(k x) f_{1}, f_{\gamma}\right) \xi\left(k^{-1}\right) \tau \bar{\chi}_{1}(\operatorname{det} k) d k S_{\gamma}(y, u)
\end{aligned}
$$

where the sum on $\gamma$ is finite (independently of $x$ ). From this the corresponding formula with $S$ replaced by $W$ follows immediately (first for $\mathscr{R} e(u)>1$, then by analytic continuation for all $u$ ); substituting it into the last formula for $J(s, u)$ we obtain

$$
\begin{aligned}
& J(s, u)=\beta(u) \int_{G_{\mathbf{A}}} \sum_{\gamma} \int_{K}\left(\pi_{\chi_{1}, \chi_{2}, u}(k x) f_{1}, f_{\gamma}\right) \xi\left(k^{-1}\right) \tau \bar{\chi}_{1}(\operatorname{det} k) d k \\
& \quad \times \int_{\mathbf{A} \times} W_{\gamma}\left[\left(\begin{array}{cc}
a & 0 \\
0 & 1
\end{array}\right), u\right] \tau \bar{\chi}_{2}(a)|\operatorname{det} a|^{s+1-u} d^{\times} a \Psi(x) \tau \bar{\chi}_{1}(\operatorname{det} x)|\operatorname{det} x|^{s+1-u} d x \\
& =\beta(u) \sum_{\gamma} V_{\gamma}(1, u ; s, \tau) C_{\gamma}(s, u),
\end{aligned}
$$

where

$$
\begin{aligned}
C_{\gamma}(s, u) & =\int_{G_{\mathbf{A}}} \int_{K}\left(\pi_{\chi_{1}, \chi_{2}, u}(k x) f_{1}, f_{\gamma}\right) \xi\left(k^{-1}\right) \tau \bar{\chi}_{1}(\operatorname{det} k x) \Psi(x)|\operatorname{det} x|^{s+1-u} d k d x \\
& =\int_{G_{\mathbf{A}}}\left(\pi_{\chi_{1}, \chi_{2}, u}(x) f_{1}, f_{\gamma}\right) \int_{K} \xi(k) \Psi(k x) d k \tau \bar{\chi}_{1}(\operatorname{det} x)|\operatorname{det} x|^{s+1-u} d x \\
& =\int_{G_{\mathbf{A}}}\left(\pi_{\chi_{1}, \chi_{2}, u}(x) f_{1}, f_{\gamma}\right) \Psi(x) \tau \bar{\chi}_{1}(\operatorname{det} x)|\operatorname{det} x|^{s+1-u} d x .
\end{aligned}
$$

Thus we have proved the representation of $J(s, u)$ as a sum which was given above and shown that the sum is finite.

Now $\beta(u)=\overline{P(1-\bar{u})} L\left(2-2 u, \bar{\chi}_{1} \chi_{2}\right)^{-1}$, where $\overline{P(1-\bar{u})}$ is the quotient of a polynomial in $u$ and finitely many $q_{v}^{ \pm u}$ by a polynomial in $u$ which does not vanish for $\mathscr{R} e(u)<1$, and we also know (by Lemma 2 of 1.2) $V_{\gamma}(1, u, s, \tau)$ equals

$$
Q_{\gamma}(s, u) L(s, \tau) L\left(s+2 u-1, \tau \chi_{1} \bar{\chi}_{2}\right) L\left(2 u, \chi_{1} \bar{\chi}_{2}\right)^{-1}
$$

where $Q_{\gamma}$ is the quotient of a polynomial in $s, u$ and finitely many $q_{v}^{ \pm s}, q_{v}^{ \pm u}$ by a polynomial in $u$, nonzero for $\mathscr{R} e(u)>0$. Therefore to prove the proposition we have 
to show that $C_{\gamma}(s, u)$ is an elementary multiple of $L(s, \tau) L\left(s+1-2 u, \tau \bar{\chi}_{1} \chi_{2}\right)$. To see this, we substitute for the scalar product $\left(\pi_{\chi_{1}, \chi_{2}, u}(x) f_{1}, f_{\gamma}\right)$ its value $\int_{K} S f_{1}(k x, u) \overline{f_{\gamma}(k)} d k$ and then make the substitution $x \rightarrow k^{-1} x$ in the resulting double integral to get

$C_{\gamma}(s, u)=\int_{G_{\mathrm{A}}} \int_{K} \Psi\left(k^{-1} x\right) \overline{f_{\gamma}(k)} \bar{\tau} \chi_{1}(\operatorname{det} k) d k S f_{1}(x, u) \tau \bar{\chi}_{1}(\operatorname{det} x)|\operatorname{det} x|^{s+1-u} d x$

Using the Iwasawa decomposition $x=\left(\begin{array}{ll}a & v \\ 0 & b\end{array}\right) k^{\prime}$ and the definition of $S f_{1}(x, u)$ we can rewrite this

$$
\begin{array}{r}
C_{\gamma}(s, u)=\int_{\mathbf{A} \times} \int_{\mathbf{A}^{\times}}\left(\int_{\mathbf{A}} \int_{K} \int_{K} \Psi\left[k^{-1}\left(\begin{array}{ll}
a & v \\
0 & b
\end{array}\right) k^{\prime}\right]\right. \\
\left.\times f_{1}\left(k^{\prime}\right) \overline{f_{\gamma}(k)} \tau \bar{\chi}_{1}\left(\operatorname{det} k^{-1} k^{\prime}\right) d k d k^{\prime} d v\right) \\
\times \tau(a)|a|^{s} \tau \bar{\chi}_{1} \chi_{2}(b)|b|^{s+1-2 u} d^{\times} a d^{\times} b,
\end{array}
$$

and since the expression in parentheses is (the restriction to $\mathbf{A} \times 2$ of) a SchwartzBruhat function of $a$ and $b$ this is a Tate integral for $L(s, \tau) L\left(s+1-2 u, \tau \chi_{1}^{-1} \chi_{2}\right)$ as claimed.

Proof of Proposition 2. We return to the formula

$$
J(s, u)=\beta(u) \sum_{\gamma} V_{\gamma}(1, u ; s, \tau) C_{\gamma}(s, u) .
$$

The function $C_{\gamma}(s, u)$ is analytic at $u=(1-s) / 2$. By Lemma 2 of 1.2 , the function $V_{\gamma}(1, u ; s, \tau)$ has a simple pole with

$$
\operatorname{Res}_{u=(1-s) / 2} V_{\gamma}(1, u ; s, \tau)=-\frac{1}{2} S f_{\gamma}(1,(1-s) / 2)=-\frac{1}{2} f_{\gamma}(1)
$$

if $\tau=\chi_{1}^{-1} \chi_{2}$ and no pole if $\tau \neq \chi_{1}^{-1} \chi_{2}$. Hence

$$
\begin{aligned}
\operatorname{Res}_{u=(1-s) / 2} J(s, u) & =-\frac{1}{2} \beta\left(\frac{1-s}{2}\right) \sum_{\gamma} f_{\gamma}(1) C_{\gamma}\left(s, \frac{1-s}{2}\right) \\
& =-\frac{1}{2} \beta\left(\frac{1-s}{2}\right) \int_{G_{\mathbf{A}}} S f_{1}\left(x, \frac{1-s}{2}\right) \Psi(x) \tau \bar{\chi}_{1}(\operatorname{det} x)|\operatorname{det} x|^{(3 s+1) / 2} d x,
\end{aligned}
$$

where in the last line we used the same identity as was used to decompose $J(s, u)$ as a sum over $\gamma$. Using the Iwasawa decomposition, we get

$$
\begin{aligned}
& -\frac{1}{2} \beta\left(\frac{1-s}{2}\right) \int_{\mathbf{A}^{\times}} \int_{\mathbf{A}^{\times}} \int_{K} \int_{N_{\mathbf{A}}} \Phi[(0, b) k] \overline{\Phi_{2}[(0, a) w n k]} \\
& \quad \times S f_{1}\left(k, \frac{1-s}{2}\right) \tau^{2}(b)|b|^{2 s} \tau(a)|a|^{1+s} \tau \chi_{1}^{-1}(\operatorname{det} k) d n d k d^{\times} a d^{\times} b .
\end{aligned}
$$


But

$$
\int_{\mathbf{A}^{\times}} \Phi[(0, b) k] \tau^{2}(b)|b|^{2 s} d^{\times} b \tau(\operatorname{det} k)=f(k, s)
$$

and (recall that $\chi_{1} \chi_{2}^{-1}=\bar{\tau}$ ),

$$
\begin{gathered}
\beta\left(\frac{1-s}{2}\right) \int_{\mathbf{A}^{\times}} \overline{\Phi_{2}[(0, a) g]} \tau(a)|a|^{1+s} \chi_{1}^{-1}(\operatorname{det} g)|\operatorname{det} g|^{(1+s) / 2} \\
=\overline{S f_{2}\left(g, \frac{1+\bar{s}}{2}\right)} .
\end{gathered}
$$

Hence our residue can be written as

$$
-\frac{1}{2} \int_{N_{\mathbf{A}}} \int_{K} S f_{1}\left(k, \frac{1-s}{2}\right) \overline{S f_{2}\left(w n k, \frac{1+\bar{s}}{2}\right)} f(k, s) d k d n,
$$

which is equivalent to the formula given in the proposition.

Proof of Proposition 3. We first observe that

$$
S \tilde{f}_{i}(g, u)=S f_{i}\left(w g^{\imath}, u\right)
$$

and hence by an easy computation

$$
W \tilde{f}_{i}(g, u)=w f_{i}\left[\left(\begin{array}{cc}
-1 & 0 \\
0 & 1
\end{array}\right) w g^{\iota}, u\right]
$$

By the lemma in 1.1 we may assume that

$$
\begin{gathered}
S f_{i}(g, u)=\alpha_{i}(u) f\left(g, \Phi_{i}, \chi_{1}, \chi_{2}, u\right), \\
W f_{i}(g, u)=\alpha_{i}(u) W f\left(g, \Phi_{i}, \chi_{1}, \chi_{2}, u\right)
\end{gathered}
$$

where $\alpha_{i}(u)$ depends only on $u$ and $\Phi_{1}, \Phi_{2}$ are Schwartz-Bruhat functions. It therefore suffices to prove the functional equation

$$
\begin{aligned}
\int_{N_{\mathbf{A}} \backslash G_{\mathbf{A}}} \Phi[(0,1) x] W f\left(x, \Phi_{1}, \chi_{1}, \chi_{2}, u\right) & \\
& \times \overline{W f\left(x, \Phi_{2}, \chi_{1}, \chi_{2}, 1-\bar{u}\right)} \tau(\operatorname{det} x)|\operatorname{det} x|^{s} d x \\
=\int_{N_{\mathbf{A}} \backslash G_{\mathbf{A}}} \hat{\Phi}[(0,1) x] W f\left[\left(\begin{array}{cc}
-1 & 0 \\
0 & 1
\end{array}\right) w x^{\iota}, \Phi_{1}, \chi_{1}, \chi_{2}, u\right] & \frac{\left(W f\left[\left(\begin{array}{cc}
-1 & 0 \\
0 & 1
\end{array}\right) w x^{\iota}, \Phi_{2}, \chi_{1}, \chi_{2}, \bar{u}\right]\right.}{\quad \times \bar{\tau}(\operatorname{det} x)|\operatorname{det} x|^{1-s} d x,}
\end{aligned}
$$

where $u$ is fixed and the equation is to be interpreted in the sense of analytic continuation in $s$. We may assume that $\Phi_{1}$ and $\Phi_{2}$ are products of local SchwartzBruhat functions $\Phi_{i v}$. Then the desired functional equation follows by multiplying together the corresponding local equations as given in [5, p. 20 or 3, p. 475] and taking into account the functional equations of the $L$-series involved. 
Proof of Proposition 4. It is clear from Proposition 1 that $J(s, u)$ has a double pole at $s=1$ only if $\tau=1$, which we now assume. The representation

$$
J(s, u)=\beta(u) \sum_{\gamma} V_{\gamma}(1, u ; s, 1) C_{\gamma}(s, u)
$$

together with the fact that $V_{\gamma}(1, u ; s, 1)$ and $C_{\gamma}(s, u)$ both have simple poles at $s=1$ implies

$$
J(s, u) \sim \frac{\beta(u)}{s-1} \sum_{\gamma} \operatorname{Res}_{s=1}\left(V_{\gamma}(1, u ; s, 1)\right) C_{\gamma}(s, u)
$$

as $s \rightarrow 1$. By Exercise 2 of 1.2, the sum on the right equals

$$
\begin{array}{r}
\sum_{\gamma} S f_{\gamma}(w, u) C_{\gamma}(s, u)=\int_{G_{\mathbf{A}}} \Psi(x) S f_{1}(w x, u) \chi_{1}^{-1}(\operatorname{det} x)|\operatorname{det} x|^{s+1-u} d x \\
=\int_{\mathbf{A}^{\times}} \int_{\mathbf{A}^{\times}}\left(\int_{\mathbf{A}} \int_{K} \Phi[(a, v) k] \overline{\Phi_{2}[(0, b) k]} \chi_{1}^{-1}(\operatorname{det} k) f_{1}(k) d v d k\right) \\
\times \chi_{1}^{-1} \chi_{2}(b)|a|^{s}|b|^{s+1-2 u} d^{\times} a d^{\times} b,
\end{array}
$$

where in the last line we have replaced $x$ by $w x$ and used the Iwasawa decomposition. By the usual argument, the residue of this at $s=1$ is obtained by replacing $\int_{\mathbf{A}} \times(\cdots)|a|^{s} d^{\times} a$ by $\int_{\mathbf{A}}(\cdots) d a$, so

$$
\begin{aligned}
\lim _{s \rightarrow 1}(s-1)^{2} J(s, u)= & \beta(u) \int_{K} \int_{\mathbf{A}^{\times}}\left(\int_{\mathbf{A}^{2}} \Phi[(a, v) k] d a d v\right) \\
& \times \overline{\Phi_{2}[(0, b) k]} \chi_{1}^{-1} \chi_{2}(b)|b|^{2-2 u} d^{\times} b \chi_{1}^{-1}(\operatorname{det} k) f_{1}(k) d k \\
= & C \beta(u) \int_{K} \overline{f\left(k, \Phi_{2}, \chi_{1}, \chi_{2}, 1-\bar{u}\right)} f_{1}(k) d k \\
= & C \int_{K} \overline{S f_{2}(k, 1-\bar{u})} f_{1}(k) d k \\
= & C \int_{K} f_{1}(k) \overline{f_{2}(k)} d k .
\end{aligned}
$$

\section{Analytic continuation and applications.}

3.1. Computation of $I(s)$ for $0<\operatorname{Res}<1$. In $\S 2$, we calculated the integral $I(s)=I(s, \varphi, \Phi, \tau)$ for $\mathscr{R} e(s)>1$ and obtained a formula of the form

$$
I(s)=\sum_{[E: F]=2} I_{E}(s)+\sum_{i=1}^{4} I_{i}(s)+\sum_{\chi} I_{\chi}(s)
$$


where the sum over $E$ and $\chi$ are finite (for a given $\varphi$ ) and

$$
\begin{aligned}
& I_{E}(s)=I_{E}(s, \varphi, \Phi, \tau) \\
& =\int_{E_{\mathbf{A}}^{\times} \backslash G_{\mathbf{A}}}\left(\frac{1}{2} \sum_{\substack{\lambda \in E^{\times} / F^{\times} \\
\lambda \neq 1}} \varphi\left(x^{-1} \lambda x\right)\right)\left(\int_{E_{\mathbf{A}}^{\times}} \Phi[(0,1) e x] \tau(\operatorname{det} e x)|\operatorname{det} e x|^{s} d^{\times} e\right) d x \\
& \sim L_{E}\left(s, \tau \circ N_{E / F}\right) \text {, } \\
& I_{1}(s)\left(=I_{\text {hyp }}(s)\right)=I_{1}(s, \varphi, \Phi, \tau) \\
& =\int_{A_{\mathbf{A}} \backslash G_{\mathbf{A}}}\left(\frac{1}{2} \sum_{\substack{\lambda \in A_{F} / Z_{F} \\
\lambda \neq 1}} \varphi\left(x^{-1} \lambda x\right)\right)\left(\int_{A_{\mathbf{A}}} \Phi[(1,1) a x] \tau(\operatorname{det} a x)|\operatorname{det} a x|^{s} d^{\times} a\right) d x \\
& \sim L_{F}(s, \tau)^{2} \text {, } \\
& I_{2}(s)\left(=I_{\text {unip }}(s)\right)=I_{2}(s, \varphi, \Phi, \tau) \\
& =\int_{K}\left(\int_{\mathbf{A}^{\times}} \varphi\left[k^{-1}\left(\begin{array}{ll}
1 & a \\
0 & 1
\end{array}\right) k\right] \tau(a)|a|^{s} d^{\times} a\right) \\
& \times\left(\int_{\mathbf{A}^{\times}} \int_{\mathbf{A}} \Phi[(t, u) k] d u|t|^{2 s-1} \tau^{2}(t) d^{\times} t\right) \tau(\operatorname{det} k) d k \\
& \sim L(s, \tau) L\left(2 s-1, \tau^{2}\right), \\
& I_{3}(s)\left(=I_{\infty}^{\prime}(s)\right)=I_{3}(s, \varphi, \Phi, \tau) \\
& =\int_{K} f(k, s) \int_{\mathbf{A}^{\times}}\left(\int_{\mathbf{A}} \varphi\left[k^{-1}\left(\begin{array}{ll}
1 & u \\
0 & 1
\end{array}\right) k\right] \psi(a u) d u\right) \tau(a)|a|^{s} d^{\times} a d k \\
& \sim L_{F}(s, \tau) L_{F}\left(2 s, \tau^{2}\right) \text {, } \\
& I_{4}(s)\left(=I_{\infty}^{\prime \prime}(s)\right)=I_{4}(s, \varphi, \Phi, \tau)=-\frac{1}{2} \sum_{\chi^{2}=\omega \tau} \int_{N_{\mathrm{A}}} \xi_{s, \chi}(w n) d n \\
& \sim \frac{L_{F}(s, \tau) L_{F}\left(2 s, \tau^{2}\right)}{L_{F}(s+1, \tau)}
\end{aligned}
$$

(finite sum) with $\xi_{s, \chi} \in H\left(\chi, \chi^{-1} \omega,(s+1) / 2\right)$ defined by

$$
\begin{aligned}
\xi_{s, \chi}(g) & =\xi(s, \varphi, \Phi, \tau, \chi, g) \\
& =\int_{\mathbf{A}^{\times}} \int_{K} f(k, s) \int_{N_{\mathbf{A}}} \varphi\left[k^{-1} n\left(\begin{array}{cc}
a^{-1} & 0 \\
0 & 1
\end{array}\right) g k\right] \chi(a)|a|^{(s+1) / 2} d k d^{\times} a d n,
\end{aligned}
$$

and

$$
\begin{aligned}
I_{\chi}(s) & =I_{\chi}(s, \varphi, \Phi, \tau) \\
& =-\frac{1}{4 \pi i} \int_{\mathscr{R}_{e}(u)=1 / 2} \sum_{\alpha, \beta \in A\left(\chi, \chi^{-1} \omega\right)}\left(\pi_{\chi, \chi^{-1} \omega, u}(\varphi) f_{\beta}, f_{\alpha}\right) J_{\alpha, \beta}(s, u) d u
\end{aligned}
$$


(finite sum) with

$$
\begin{aligned}
J_{\alpha, \beta}(s, u) & =\int_{N_{\mathbf{A}} \backslash G_{\mathbf{A}}} \Phi[(0,1) x] W f_{\alpha}(x, u) \overline{W f_{\beta}(x, 1-\bar{u})} \tau(\operatorname{det} x)|\operatorname{det} x|^{s} d x \\
& \sim \frac{L_{F}(s, \tau)^{2} L_{F}\left(s+1-2 u, \bar{\chi}^{2} \omega \tau\right) L_{F}\left(s-1+2 u, \chi^{2} \omega^{-1} \tau\right)}{L_{F}\left(2-2 u, \bar{\chi}^{2} \omega\right) L_{F}\left(2 u, \chi^{2} \omega^{-1}\right)}
\end{aligned}
$$

(in all of these formulas, $f(s) \sim L(s)$ means that $f$ is an entire multiple of $L$ ).

We define one more function $I_{5}(s)$ by

$$
I_{5}(s)=I_{5}(s, \varphi, \Phi, \tau)=I_{4}\left(1-s, \varphi^{\iota}, \hat{\Phi}, \bar{\tau}\right),
$$

where $\varphi^{\imath}(x)=\varphi\left(x^{\imath}\right)$.

THEOREM 1. With the notations above the function $I(s)$ is given in the strip $0<\mathscr{R}_{e}(s)<1$ by the formula

$$
I(s)=\sum_{E} I_{E}(s)+\sum_{i=1}^{5} I_{i}(s)+\sum_{\chi} I_{\chi}(s) .
$$

In the half-plane $\mathscr{R}_{e}(s)>1$ (resp. $\left.\mathscr{R e}(s)<0\right), I(s)$ is given by the same formula but without the term $I_{5}(s)$ (resp. $I_{4}(s)$ ). The substitution $s \rightarrow 1-s, \tau \rightarrow \bar{\tau}, \Phi \rightarrow \hat{\Phi}$, $\varphi \rightarrow \varphi^{\imath}$, under which $I(s)$ is invariant, interchanges $I_{2}$ and $I_{3}$, interchanges $I_{4}$ and $I_{5}$, and leaves all the other terms unchanged.

COROllaRy. The quotient $I(s) / L_{F}(s, \tau)$ is entire, except for simple poles at $s=0$ and $s=1$ if $\tau^{2}=1, \tau \neq 1$.

Proof. The original definition of $I(s)$ as an integral shows that it is an entire function of $s$ except for simple poles at $s=0$ and 1 if $\tau^{2}=1$. To prove Theorem 1 and its corollaries we have to investigate the analytic continuation of each term in the formula we have obtained for $I(s), \mathscr{R}_{e}(s)>1$. The only terms for which this is not immediate are the terms $I_{\chi}(s)$.

Recall that $I_{\chi}(s)$ is given as an integral of the form

$$
I_{\chi}(s)=\frac{1}{2 \pi i} \int_{L} I_{\chi}(s, u) d u
$$

where $L$ denotes the line $\mathscr{R} e(u)=\frac{1}{2}$ and $I_{\chi}(s, u)$ has the form

$$
I_{\chi}(s, u)=L_{F}(s, \tau)^{2} \frac{A(s, u)}{D(u)}
$$

where $D(u)$ is a meromorphic function of $u$ having no zeroes on the line $L(D(u)$ equals $L\left(2-2 u, \bar{\chi}^{2} \omega\right) L\left(2 u, \chi^{2} \omega^{-1}\right)$ times the product of the functions $P(u)$ occurring in the proposition of 2.4 for $\left.f_{1}=f_{\alpha}, f_{2}=f_{\beta}\right)$ and $A(s, u)$ is holomorphic in $s$ and $u$ except for possible poles at the points

$$
u=\frac{s}{2}, \quad 1-\frac{s}{2}, \frac{1+s}{2}, \frac{1-s}{2},
$$


where it has poles if $\chi^{-2} \omega=\tau^{ \pm 1}$, these poles being simple unless two of the numbers (*) coincide. The integral $\int_{L} A(s, u) / D(u) d u$ converges for all $s \in \mathbb{C}$ except that it does not make sense if $\mathscr{R} e(s)=0$ or 1 because at least one of the poles (*) lies on $L$. It therefore defines a holomorphic function of $s$ in each of the three regions

$$
\begin{aligned}
& R_{0}: \mathscr{R}_{e}(s)>1, \\
& R_{1}: 0<\mathscr{R}_{e}(s)<1, \\
& R_{2}: \mathscr{R}_{e}(s)<0 .
\end{aligned}
$$

However, the function it defines in these three regions are not analytic continuations of one another.

To obtain the analytic continuation, let $B$ be a box in the $u$-plane with center at $u=\frac{1}{2}$ and sides parallel to the axes which does not contain any zeroes of $D(u)$ and let $L^{\prime}$ be the deformation of $L$ obtained by going along $L$, then around the right edge of the box $B$, and then continuing along $L$. See Figure 2 . Then for the same reason as before, the integral $\int_{L^{\prime}} A(s, u) / D(u) d u$ makes sense for all $s \in \mathbb{C}$ for which none of the points (*) lies on $L^{\prime}$ and in particular for all $s$ in the interior of the box $B^{\prime}=2 B=\left\{s \mid \frac{1}{2} s \in B\right\}$. To obtain the analytic continuation of the integral over $L$ from $R_{0}$ to $R_{1}$, we will compare this integral with the integral over $L^{\prime}$ in the right and left halves of the box $B^{\prime}$. See Figure 3. For $s \in R_{0} \cap B^{\prime}$ we have

$$
\frac{1}{2 \pi i}\left(\int_{L^{\prime}}-\int_{L}\right) \frac{A(s, u)}{D(u)} d u=\frac{1}{D(s / 2)} \operatorname{Res}_{u=s / 2} A(s, u)
$$

by Cauchy's theorem, since the only pole of the integrand between $L$ and $L^{\prime}$ is at $s / 2$. Similarly, for $s \in R_{1} \cap B^{\prime}$ we find

$$
\frac{1}{2 \pi i}\left(\int_{L^{\prime}}-\int_{L}\right) \frac{A(s, u)}{D(u)} d u=\frac{1}{D(1-s / 2)} \operatorname{Res}_{u=1-s / 2} A(s, u) .
$$

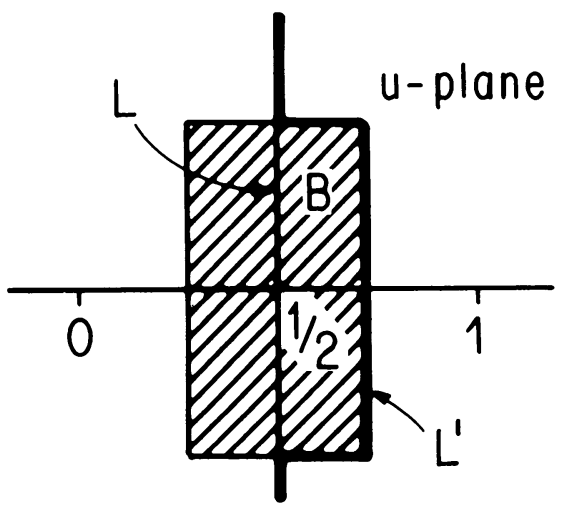

FIGURE 2 


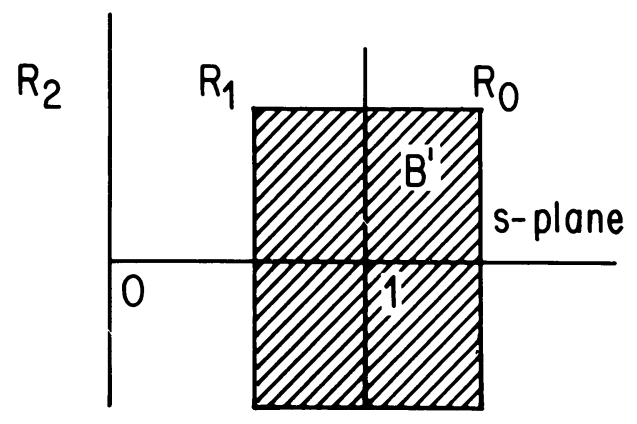

FIgURE 3

Together, these formulas give the analytic continuation of $I_{\chi}(s)$ to the region $R_{0} \cup R_{1} \cup B^{\prime}:$

$$
I_{\chi}(s)= \begin{cases}\frac{1}{2 \pi i} \int_{L} I_{\chi}(s, u) d u & \left(s \in R_{0}\right), \\ \frac{1}{2 \pi i} \int_{L^{\prime}} I_{\chi}(s, u) d u-\operatorname{Res}_{u=s / 2} I_{\chi}(s, u) & \left(s \in B^{\prime}\right), \\ \frac{1}{2 \pi i} \int_{L} I_{\chi}(s, u) d u-\left(\operatorname{Res}_{u=s / 2}-\operatorname{Res}_{u=1-s / 2}\right) I_{\chi}(s, u) & \left(s \in R_{1}\right) .\end{cases}
$$

Since we can take the box $B$ (and hence $B^{\prime}$ ) to be as tall as we want by making it thin enough, this in fact provides the analytic continuation of $I_{\chi}(s)$ to the entire half-plane $\mathscr{R}_{e}(s)>0$. (The continuation to the entire plane could then be obtained by the same method, taking for $B$ a box with center $u=0$, but since we will prove functional equations for all the terms in the formula for $I(s)$ it will not be necessary to carry this out.)

We will now compute the residues of $I_{\chi}(s, u)$ at $u=s / 2$ and $u=1-s / 2$, obtaining

$$
\operatorname{Res}_{u=s / 2} I_{\chi}(s, u)=-\operatorname{Res}_{u=1-s / 2} I_{\chi}(s, u)=-\frac{1}{2} I_{5}(s)
$$

where $I_{5}(s)$ is the function defined just before Theorem 1; this and the above discussion then give the formula for $I(s)\left(s \in R_{1}\right)$ which was stated in the theorem.

To compute the residues of $I_{\chi}(s, u)$ we use the formulas for the residues of $J(s, u)$ given after the statement of Proposition 3 of 2.5. Substituting the first of these formulas into the definition of $I_{\chi}(s, u)$ gives

$$
\begin{aligned}
\operatorname{Res}_{u=s / 2} I_{\chi}(s, u)= & -\frac{1}{4} \sum_{\alpha, \beta \in A\left(\chi, \chi^{-1} \omega\right)}\left(\pi_{\chi, \chi^{-1} \omega, s / 2}(\varphi) f_{\beta}, f_{\alpha}\right) \\
& \times \int_{A_{\mathbf{A}} \backslash G_{\mathbf{A}}} S \tilde{f}_{\alpha}\left(x, \frac{s}{2}\right) \overline{S \tilde{f}_{\beta}\left(w x, 1-\frac{\bar{s}}{2}\right)} f^{\prime}(x, s) d x
\end{aligned}
$$


if $\chi^{2}=\omega \tau$ (the residue is 0 if $\chi^{2} \neq \omega \tau$ ). But

$$
\begin{aligned}
\sum_{\alpha}\left(\pi_{\chi, \chi^{-1} \omega, s / 2}(\varphi) f_{\beta}, f_{\alpha}\right) S \tilde{f}_{\alpha}(x, s / 2) & =\sum_{\alpha}\left(\pi_{\chi \omega^{-1}, \chi^{-1}, s / 2}\left(\varphi^{\iota}\right) \tilde{f}_{\beta}, \tilde{f}_{\alpha}\right) S \tilde{f}_{\alpha}(x, s / 2) \\
& =\int_{G_{\mathbf{A}}} S \tilde{f}_{\beta}(h, s / 2) \varphi^{\iota}\left(x^{-1} h\right) d h,
\end{aligned}
$$

where $\varphi^{\imath}(x)=\varphi\left(x^{\imath}\right)$. Substituting this into the formula for the residue and computing the integral over $h$ by the Iwasawa decomposition, we find that the residue equals

$$
-\frac{1}{4} \sum_{\beta} \int_{A_{\mathbf{A}} \backslash G_{\mathbf{A}}} \int_{K} S \tilde{f}_{\beta}\left(k, \frac{s}{2}\right) \overline{S \tilde{f}_{\beta}\left(w x, 1-\frac{\bar{s}}{2}\right)} F_{x, \chi}(k) f^{\prime}(x, s) d k d x,
$$

where we have set

$$
F_{x, \chi}(h)=\int_{N_{\mathbf{A}}} \int_{\mathbf{A}^{\times}} \varphi^{\iota}\left[x^{-1} n\left(\begin{array}{ll}
a & 0 \\
0 & 1
\end{array}\right) h\right] \chi \omega^{-1}(a)|a|^{s / 2-1} d^{\times} a d n .
$$

Note that $h \rightarrow F_{x, \chi}(h)$ belongs to $H\left(\chi^{-1} \omega, \chi, 1-s / 2\right)$, as does the function $h$

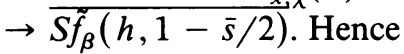

$$
\sum_{\beta} \int_{K} S \tilde{f}_{\beta}\left(k, \frac{s}{2}\right) \overline{S \tilde{f}_{\beta}\left(w x, 1-\frac{\bar{s}}{2}\right)} F_{x, \chi}(k) d k=F_{x, \chi}(w x),
$$

so we obtain

$$
\begin{aligned}
\operatorname{Res}_{u=s / 2} I_{\chi}(s, u)= & -\frac{1}{4} \int_{K} \int_{N_{\mathbf{A}}} \int_{N_{\mathbf{A}}} \int_{A_{\mathbf{A}}} \varphi^{i}\left[k^{-1} n_{1}\left(\begin{array}{cc}
a^{-1} & 0 \\
0 & 1
\end{array}\right) w n_{2} k\right] \\
& \times \chi^{-1} \omega(a)|a|^{1-s / 2} f^{\prime}(k, s) d^{\times} a d n_{1} d n_{2} d k \\
= & -\frac{1}{4} \int_{N_{\mathbf{A}}} \xi\left(1-s, \varphi^{\iota}, \hat{\Phi}, \tau^{-1}, \chi^{-1} \omega, w n\right) d n
\end{aligned}
$$

(with $\xi(\cdots)$ defined as at the beginning of the chapter) and

$$
\begin{aligned}
\sum_{\chi} \operatorname{Res}_{u=s / 2} I_{\chi}(s, u) & =\sum_{\chi^{2}=\omega \tau} \operatorname{Res}_{u=s / 2} I_{\chi}(s, u) \\
& =\frac{1}{2} I_{4}\left(1-s, \varphi^{\iota}, \hat{\Phi}, \tau^{-1}\right) \\
& =\frac{1}{2} I_{5}(s, \varphi, \Phi, \tau) .
\end{aligned}
$$

To obtain the other residue, we use the trivial functional equations

$$
J\left(f_{1}, f_{2} ; \Phi, \tau ; s, u\right)=\overline{J\left(f_{2}, f_{1} ; \bar{\Phi}, \tau^{-1} ; \bar{s}, 1-\bar{u}\right)}
$$

and

$$
\left(\pi_{\chi, \chi^{-1} \omega, u}(\varphi) f_{\beta}, f_{\alpha}\right)=\overline{\left(\pi_{\chi, \chi^{-1} \omega, 1-\bar{u}}\left(\overline{\varphi^{\vee}}\right) f_{\alpha}, f_{\beta}\right)}
$$

(where $\varphi^{\vee}(x)=\varphi\left(x^{-1}\right)$; this formula can be obtained easily by applying the Iwasawa decomposition to the integral defining the scalar product) to get

$$
I_{\chi}(s, u ; \varphi, \Phi, \tau)=\overline{I_{\chi}\left(\bar{s}, 1-\bar{u} ; \overline{\varphi^{\vee}}, \bar{\Phi}, \bar{\tau}\right)}
$$


and hence

$$
\begin{aligned}
\sum_{\chi^{2}=\omega \bar{\tau}} \operatorname{Res}_{u=1-s / 2} I_{\chi}(s, u ; \varphi, \Phi, \tau) & =-\sum_{\chi^{2}=\omega \bar{\tau}} \overline{\operatorname{Res}_{u=\bar{s} / 2} I_{\chi}\left(\bar{s}, u, \overline{\varphi^{\vee}}, \bar{\Phi}, \bar{\tau}\right)} \\
& =-\frac{1}{2} \overline{I_{5}\left(\bar{s}, \overline{\varphi^{\vee}}, \bar{\Phi}, \bar{\tau}\right)}
\end{aligned}
$$

To see that this last expression equals $-\frac{1}{2} I_{5}(s, \varphi, \Phi, \tau)$, we have to show that $I_{5}$ (or $I_{4}$ ) is invariant under $\varphi \rightarrow \varphi^{\vee}$. We recall the definition of $I_{4}$,

$$
\begin{array}{r}
I_{4}(s, \varphi, \Phi, \tau)=-\frac{1}{2} \sum_{\chi^{2}=\bar{\omega} \tau} \int_{N_{\mathbf{A}}} \int_{\mathbf{A} \times} \int_{K} \int_{N_{\mathbf{A}}} f(k, s) \varphi\left[k^{-1} n\left(\begin{array}{ll}
a^{-1} & 0 \\
0 & 1
\end{array}\right) w n^{\prime} k\right] \\
\times \chi(a)|a|^{(s+1) / 2} d n d k d^{\times} a d n^{\prime} .
\end{array}
$$

Replacing $\varphi$ by $\varphi^{\vee}$ in this formula (note that this replaces $\omega$, the central character of $\varphi$, by $\omega^{-1}$ ) and using the identity

$$
\varphi^{\vee}\left[k^{-1} n\left(\begin{array}{ll}
a^{-1} & 0 \\
0 & 1
\end{array}\right) w n^{\prime} k\right]=\omega(a) \varphi\left[k^{-1} n^{\prime-1}\left(\begin{array}{ll}
a^{-1} & 0 \\
0 & 1
\end{array}\right) w n^{-1} k\right]
$$

we find (after the change of variables $\left.n \rightarrow n^{\prime-1}, n^{\prime} \rightarrow n^{-1}, \chi \rightarrow \chi \omega\right)$ that $I_{4}(s, \varphi, \Phi, \tau)$ equals $I_{4}\left(s, \varphi^{\vee}, \Phi, \tau\right)$.

It remains to investigate the effect of the substitutions $s \rightarrow 1-s, \varphi \rightarrow \varphi^{l}$, $\Phi \rightarrow \hat{\Phi}, \tau \rightarrow \tau^{-1}$ on the various terms in the formula for $I(s)$. These interchange $I_{4}$ and $I_{5}$ by definition. We consider the other terms

$I_{E}(s)$. The invariance of $I_{E}(s)$ under the substitution in question follows by the usual Tate method for proving the functional equations of $L$-functions if we identify $E_{A}$ with $A^{2}$ and observe that the Haar measures and additive characters on these two spaces agree. In any case, this functional equation is evident a priori since $I_{E}(s)$ is the integral of the $G_{F}$-invariant function $\sum_{\lambda \in G_{F} ; F[\lambda] \cong E} \varphi\left(x^{-1} \lambda x\right)$ against $E\left(x, \Phi, \tau, \tau^{-1}, s\right)$.

$I_{1}(s)$. Again the invariance follows easily by applying the Tate method to the inner integral

$$
\tau(\operatorname{det} x)|\operatorname{det} x|^{s} \int_{\mathbf{A}^{\times}} \int_{\mathbf{A}^{\times}} \Phi[(a, b) x] \tau(a)|a|^{s} \tau(b)|b|^{s} d^{\times} a d^{\times} b .
$$

$I_{2}(s), I_{3}(s)$. The fact that these integrals are exchanged by the substitution in question is again a corollary of the Tate functional equation since the SchwartzBruhat function

$$
a \mapsto \int_{A} \varphi\left[k^{-1}\left(\begin{array}{cc}
1 & u \\
0 & 1
\end{array}\right) k\right] \psi(a u) d u
$$


occurring in the definition of $I_{3}$ is the Fourier transform of the function $a \mapsto$ $\varphi\left[k^{-1}\left(\begin{array}{ll}1 & a \\ 0 & 1\end{array}\right) k\right]$ occurring in $I_{2}$ and

$$
\begin{aligned}
\int_{\mathbf{A}^{\times}} \int_{\mathbf{A}} \Phi[(t, u) k] d u|t|^{2 s-1} \tau^{2}(t) d^{\times} t & =\int_{\mathbf{A}^{\times}} \widetilde{k \Phi}[(t, 0)]|t|^{2 s-1} \tau^{2}(t) d^{\times} t \\
& =\int_{\mathbf{A}^{\times}} \widehat{k \Phi}[(t, 0)]|t|^{2-2 s} \tau^{-2}(t) d^{\times} t \\
& =f\left(w k^{\iota}, \hat{\Phi}, \tau^{-1}, 1-s\right) .
\end{aligned}
$$

$\sum_{\chi} \int I_{\chi}(s, u) d u$. We have

$$
\begin{aligned}
I_{\chi \omega^{-1}}\left(1-s, \varphi^{\iota}, \hat{\Phi}, \bar{\tau}, u\right) & \\
& =\frac{1}{2} \sum_{\alpha, \beta \in A\left(\chi \omega^{-1} \cdot \chi^{-1}\right)}\left(\pi_{\chi \omega^{-1} \cdot \chi^{-1}, u}\left(\varphi^{\iota}\right) f_{\beta}, f_{\alpha}\right) J\left(f_{\alpha}, f_{\beta} ; \hat{\Phi}, \bar{\tau}, 1-s, u\right) \\
& =\frac{1}{2} \sum_{\alpha, \beta \in A\left(\chi \omega^{-1} \cdot \chi^{-1}\right)}\left(\pi_{\chi \omega^{-1} \cdot \chi^{-1}, u}\left(\varphi^{\iota}\right) f_{\beta}, f_{\alpha}\right) J\left(\tilde{f}_{\alpha}, \tilde{f}_{\beta} ; \Phi, \tau ; s, u\right)
\end{aligned}
$$

by Proposition 2 of 2.4. Since $\left(\tilde{f}_{\alpha}\right)_{\alpha \in A\left(\chi \omega^{-1} \cdot \chi^{-1}\right)}$ is a basis of $H\left(\chi, \chi^{-1} \omega\right)$ and (as is easily checked)

$$
\left(\pi_{\chi \omega^{-1} \cdot \chi^{-1}, u}\left(\varphi^{\iota}\right) f_{\beta}, f_{\alpha}\right)=\left(\pi_{\chi, \chi^{-1} \omega, u}(\varphi) \tilde{f}_{\beta}, \tilde{f}_{\alpha}\right)
$$

this gives

$$
I_{\chi \omega^{-1}}\left(1-s, \varphi^{\iota}, \hat{\Phi}, \bar{\tau}, u\right)=I_{\chi}(s, \varphi, \Phi, \tau, u)
$$

and hence the desired invariance of $\sum_{\chi} \int I_{\chi}(s, u) d u$.

This completes the proof of Theorem 1 . We still have to prove the corollary on the holomorphy of $I(s) / L(s, \tau)$. Looking at the analytic behavior of the various terms $I_{i}(s), I_{E}(s), \int I_{\chi}(s, u) d u$ in Theorem 1, we see that the quotient of each of these terms is holomorphic except for possible simple poles at $s=0,1$ and $\frac{1}{2}$ (for example, $I_{E}(s) \sim L_{E}\left(s, \tau \circ N_{E / F}\right)=L(s, \tau) L\left(s, \tau \chi_{E}\right)$ where $\chi_{E}$ is the quadratic character associated to the extension $E$ ). It remains to investigate these three points.

If $\tau^{2} \neq 1$, then the definition of $I(s)$ as an integral shows that it is regular at $s=0$ and $s=1$, and since $L(s, \tau)$ is nonzero at these two points the quotient is also regular. If $\tau=1$, then both $I(s)$ and $L(s, \tau)$ have simple poles at 0 and 1 and the quotient is again regular there. If $\tau \neq 1$ but $\tau^{2}=1$, then $I(s)$ has a simple pole and $L(s, \tau) \neq 0$, so the quotient also has a simple pole.

As to the point $s=\frac{1}{2}$, the only terms whose quotient by $L(s, \tau)$ might have a pole are $I_{2}, I_{3}, I_{4}$ and $I_{5}$, only if $\tau^{2}=1$.

Near $s=\frac{1}{2}$ we have

$$
\begin{gathered}
\tau(\operatorname{det} k) \int_{\mathbf{A}^{\times}} \int_{\mathbf{A}} \Phi[(t, u) k] d u|t|^{2 s-1} d^{\times} t=-\frac{C(k)}{s-1 / 2}+O(1), \\
C(k)=\frac{1}{2} \tau(\operatorname{det} k) \int_{\mathbf{A}} \Phi[(0, u) k] d u
\end{gathered}
$$

and hence

$$
I_{2}(s)=-\frac{1}{s-1 / 2} \int_{K} C(k) \int_{\mathbf{A}^{\times}} \varphi\left[k^{-1}\left(\begin{array}{cc}
1 & a \\
0 & 1
\end{array}\right) k\right] \tau(a)|a|^{s} d^{\times} a d k+\cdots
$$


where "..." denotes a function whose quotient by $L(s, \tau)$ is regular at $s=\frac{1}{2}$. The inner integral is for each $k$ an entire multiple of $L(s, \tau)$ and hence has the form

$$
L(s, \tau)\left(M(k)+O\left(s-\frac{1}{2}\right)\right) \quad\left(s \rightarrow \frac{1}{2}\right),
$$

so

$$
I_{2}(s)=-\frac{L(s, \tau)}{s-1 / 2} \int_{K} C(k) M(k) d k+\cdots
$$

Similarly

$$
f(k, s)=\frac{C(k)}{s-1 / 2}+O(1) \quad\left(s \rightarrow \frac{1}{2}\right)
$$

implies

$$
\begin{aligned}
I_{3}(s) & =\frac{1}{s-1 / 2} \int_{K} C(k) \int_{\mathbf{A}^{\times}} \int_{\mathbf{A}} \varphi\left[k^{-1}\left(\begin{array}{ll}
1 & u \\
0 & 1
\end{array}\right) k\right] \psi(a u) d u \tau(a)|a|^{s} d^{\times} a+\cdots \\
& =\frac{1}{s-1 / 2} \int_{K} C(k) \int_{\mathbf{A}^{\times}} \varphi\left[k^{-1}\left(\begin{array}{cc}
1 & a \\
0 & 1
\end{array}\right) k\right] \tau(a)|a|^{1-s} d^{\times} a d k+\cdots
\end{aligned}
$$

(by the usual functional equation and because $\tau=\tau^{-1}$ )

$$
\begin{aligned}
& =\frac{1}{s-1 / 2} \int_{K} C(k) L(1-s, \tau)\left(M(k)+O\left(s-\frac{1}{2}\right)\right) d k+\cdots \\
& =\frac{L(s, \tau)}{s-1 / 2} \int_{K} C(k) M(k) d k+\cdots .
\end{aligned}
$$

Hence $\left(I_{2}(s)+I_{3}(s)\right) / L(s, \tau)$ is regular at $s=\frac{1}{2}$.

A similar argument works for $I_{4}$ and $I_{5}$. Near $s=\frac{1}{2}$ we have

$$
\begin{aligned}
& I_{4}(s)=-\frac{1}{4} \sum_{\chi^{2}=\omega^{-1} \tau} \int_{K}\left(\frac{C(k)}{s-1 / 2}+O(1)\right) \\
& \quad \times\left(\int_{N_{\mathbf{A}}} \int_{\mathbf{A}^{\times}} \int_{N_{\mathbf{A}}} \varphi\left[k^{-1} n_{1}\left(\begin{array}{cc}
a^{-1} & 0 \\
0 & 1
\end{array}\right) w n_{2} k\right] \chi(a)|a|^{(s+1) / 2} d n_{1} d^{\times} a d n_{2}\right)_{\text {anal.cont. }} d k
\end{aligned}
$$

and $I_{5}$ is given by a similar expression except that $\Phi$ is replaced by $\hat{\Phi}$ (this changes $C(k)$ to $\left.C\left(w k^{\imath}\right)\right), s$ by $1-s$ and $\varphi$ by $\varphi^{c}$; using the identity

$$
\varphi^{\iota}\left[\left(w k^{\iota}\right)^{-1} n_{1}\left(\begin{array}{cc}
a^{-1} & 0 \\
0 & 1
\end{array}\right) w n_{2}\left(w k^{\iota}\right)\right]=\omega(a) \varphi\left[k^{-1} n_{1}^{-1}\left(\begin{array}{cc}
a^{-1} & 0 \\
0 & 1
\end{array}\right) w n_{2}^{-1} k\right],
$$

we again deduce the divisibility of $I_{4}+I_{5}$ by $L(s, \tau)$ at $s=\frac{1}{2}$.

3.2. Residue at $s=1$ : The Selberg trace formula. Looking at the various terms in the formula we have obtained for $I(s)$, we see that each term is regular at $s=1$ if $\tau^{2} \neq 1$. Of course, the regularity of $I(s)$ itself in this case is clear since $E(x, s)$ has no pole at $s=1$.

If on the other hand $\tau^{2}=1$, then $E(x, s)$ has a simple pole with residue $(C / 2) \tau(\operatorname{det} \chi)$ at $s=1$, where

$$
C=\hat{\Phi}(0)=\iint_{\hat{A}^{2}} \Phi(u, v) d u d v
$$


so we have

$$
\int_{G_{F} Z_{\mathbf{A}} \backslash G_{\mathbf{A}}} K_{0}(g, g) \tau(\operatorname{det} g) d g=\frac{2}{C} \operatorname{Res}_{s=1} I(s) .
$$

From the formula for $I(s)$ near $s=1$ given in 3.1, we see that this equals

$$
\begin{aligned}
\frac{2}{C}\left(\sum_{E} \operatorname{Res}_{s=1} I_{E}(s)+\sum_{i=1}^{4} \operatorname{Res}_{s=1} I_{i}(s)\right. & +\frac{1}{2} \operatorname{Res}_{s=1} I_{5}(s) \\
& \left.+\frac{1}{2 \pi i} \sum_{\chi} \int_{L^{\prime}} \operatorname{Res}_{s=1} I_{\chi}(s, u) d u\right),
\end{aligned}
$$

where $L^{\prime}$ is the line $\mathscr{R} e(u)=\frac{1}{2}$ deformed slightly so as to pass the point $u=\frac{1}{2}$ to the right (cf. Figure 2). So there are seven residues to compute. We distinguish two cases, according as $\tau$ is trivial or not.

Case 1. $\tau^{2}=1, \tau \neq 1$.

Here the terms $I_{1}(s), I_{3}(s), I_{4}(s), \int_{L^{\prime}} I_{\chi}(s, u) d u$ (all $\chi$ ) and all $I_{E}(s)$ with $\tau \circ N_{E / F} \neq 1$ (i.e. all but one $E$ ) are regular at $s=1$, while the three remaining terms all have simple poles. The residues are calculated as follows.

Let $E$ be the quadratic extension of $F$ associated to the quadratic character $\tau$, so that $\tau \circ N_{E / F}=1$. Then the inner integral in the definition of $I_{E}(s)$ has residue $C \tau(\operatorname{det} x)$ at $s=1$, so

$$
\frac{2}{C} \operatorname{Res}_{s=1} I_{E}(s)=\int_{E_{\mathbf{A}}^{\times} \backslash G_{\mathbf{A}}} \sum_{\substack{\lambda \in E^{\times} / F^{\times} \\ \lambda \neq 1}} \varphi\left(x^{-1} \lambda x\right) \tau(\operatorname{det} x) d x .
$$

Similarly, the residue at $s=1$ of the double integral over $t$ and $u$ in the definition of $I_{2}(s)$ is $C / 2$ (independent of $k$ ), so

$$
\frac{2}{C} \operatorname{Res}_{s=1} I_{2}(s)=\left.\int_{K} \int_{\mathbf{A}^{\times}} \varphi\left[k^{-1}\left(\begin{array}{cc}
1 & a \\
0 & 1
\end{array}\right) k\right] \tau(a)|a|^{s} d^{\times} a \tau(\operatorname{det} k) d k\right|_{s=1}
$$

(the integral converges for $\mathscr{R} e(s)>1$ and is a holomorphic multiple of $L(s, \tau)$, so this "contains" $L(1, \tau))$. Finally, we have

$$
\operatorname{Res}_{s=1} I_{5}(s)=-\operatorname{Res}_{s=0} I_{4}\left(s, \varphi^{\imath}, \hat{\Phi}, \tau\right) .
$$

Looking at the formula for $I_{4}$, we see that the pole at $s=0$ comes from the function $f(k, s)$ in the integrand. Since

$$
\operatorname{Res}_{s=0} f(k, \hat{\Phi}, \tau, \tau, s)=-\frac{1}{2} \hat{\Phi}(0) \tau(\operatorname{det} k),
$$

this means that the value of ${ }_{C}^{1} \operatorname{Res}_{s=1} I_{5}(s)$ is obtained by replacing $f(k, s)$ by $\frac{1}{2} \tau(\operatorname{det} k)$ in the formula for $I_{4}\left(s, \varphi^{\imath}, \hat{\Phi}, \tau\right)$ and evaluating the resulting function at $s=0$ (in the sense of analytic continuation), i.e. it equals

$$
\begin{aligned}
&-\frac{1}{4} \sum_{\chi^{2}=\omega \tau} \int_{N_{\mathbf{A}}} \int_{N_{A}} \int_{\mathbf{A}^{\times}} \int_{K} \tau(\operatorname{det} k) \varphi\left[k^{-1} n_{1}\left(\begin{array}{cc}
a^{-1} & 0 \\
0 & 1
\end{array}\right) w n_{2} k\right] \\
& \times\left.\chi(a)|a|^{s} d k d^{\times} a d n_{1} d n_{2}\right|_{s=1 / 2} .
\end{aligned}
$$


This can be interpreted as a trace in the following way: for each $\chi$ with $\chi^{2}=\omega \tau$ consider the composite operator

$$
\begin{aligned}
A_{\tau, \chi, s}(\varphi): H(\tau \chi, \chi) & \stackrel{\cong}{\rightarrow} H(\tau \chi, \chi, s) \stackrel{\pi_{\tau \chi \chi, \times, s}(\varphi)}{\rightarrow} H(\tau \chi, \chi, s) \\
& \stackrel{\tau^{\text {odet }}}{\rightarrow} H(\chi, \tau \chi, s) \stackrel{M(\chi, \tau \chi, s)}{\rightarrow} H(\tau \chi, \chi, 1-s) \stackrel{\cong}{\leftarrow} H(\tau \chi, \chi) .
\end{aligned}
$$

It is given by the kernel function

$\tau\left(\operatorname{det} k_{1}\right) \int_{N_{\mathbf{A}}} \int_{N_{\mathbf{A}}} \int_{\mathbf{A}^{\times}} \varphi\left[k_{1}^{-1} n_{1}\left(\begin{array}{cc}a^{-1} & 0 \\ 0 & 1\end{array}\right) w n_{2} k_{2}\right] \chi(a)|a|^{s} d^{\times} a d n_{1} d n_{2}$

$$
\left(k_{1}, k_{2} \in K\right),
$$

so its trace at $s=\frac{1}{2}$ is the multiple integral above. Summarizing, we have proved

THEOREM 2. Let $\tau$ be a character of order 2 on $\mathbf{A}_{F}^{\times} / F^{\times}$and $E$ the corresponding quadratic extension of $F$. Then

$$
\begin{aligned}
\int_{G_{F} Z_{\mathbf{A}} \backslash G_{\mathbf{A}}} K_{0}(g, g) \tau(\operatorname{det} g) d g & =\int_{E_{\mathbf{A}}^{\times} \backslash G_{\mathbf{A}}} \sum_{\substack{\lambda \in E^{\times} / F^{\times} \\
\lambda \neq 1}} \varphi\left(x^{-1} \lambda x\right) \tau(\operatorname{det} x) d x \\
& +\lim _{s \rightarrow 1} \int_{K} \int_{\mathbf{A}^{\times}} \varphi\left[k^{-1}\left(\begin{array}{ll}
1 & a \\
0 & 1
\end{array}\right) k\right] \tau(a)|a|^{s} d^{\times} a \tau(\operatorname{det} k) d k \\
& -\frac{1}{4} \sum_{\chi^{2}=\tau \omega} \operatorname{tr} A_{\tau, \chi}(\varphi),
\end{aligned}
$$

where $A_{\tau, \chi}(\varphi)$ is defined as the composition

$$
H\left(\tau \chi, \chi, \frac{1}{2}\right) \stackrel{\pi(\varphi)}{\rightarrow} H\left(\tau \chi, \chi, \frac{1}{2}\right) \stackrel{\cdot \tau \circ \operatorname{det}}{\rightarrow} H\left(\chi, \tau \chi, \frac{1}{2}\right) \stackrel{M\left(\chi, \tau \chi, \frac{1}{2}\right)}{\rightarrow} H\left(\tau \chi, \chi, \frac{1}{2}\right) .
$$

We have written out the result in full detail because it is, if known at all, in any case less well known than the formula for $\int K_{0}(g, g) d g$ without any character. However, we hasten to add that the integral $\int K_{0}(g, g) \tau(\operatorname{det} g) d g$ could be calculated directly (indeed, considerably more easily than in the usual case $\tau=1$, just as our residue calculation was simpler than for $\tau=1$; in fact the calculation can be carried out for $\mathrm{GL}(3)$ or even for $\mathrm{GL}(p), p$ prime [6]). We indicate how Theorem 2 can be reinterpreted as essentially equivalent to a theorem of Labesse and Langlands [8]; however, we will be brief since this is not too different from the route followed in Labesse [7].

It is shown in [8] that one can find a "smoothing function" $m: E_{\mathbf{A}}^{\times} \backslash F_{\mathbf{A}}^{\times} \rightarrow \mathbb{C}$, with $m(\lambda)=1$ for $\lambda \in E^{\times} \backslash F^{\times}$and $m(a b)=m(a) \tau(b)$ for $b \in F_{\mathbf{A}}^{\times}, a \in E_{\mathbf{A}}^{\times} \backslash F_{\mathbf{A}}^{\times}$, such that the function

$$
f(\lambda)=m(\lambda) \int_{E_{\mathbf{A}}^{\times} \backslash G_{\mathbf{A}}} \varphi\left(x^{-1} \lambda x\right) \tau(\operatorname{det} x) d x,
$$

a priori defined only for $\lambda \in E_{\mathbf{A}}^{\times} \backslash F_{\mathbf{A}}^{\times}$, extends to a smooth function on $E_{\mathbf{A}}^{\times}$with $f(1)$ equal to the term $\lim _{s \rightarrow 1} \cdots$ in Theorem 2. Then the first two terms on the right-hand side of the theorem equal

$$
\sum_{\lambda \in E^{\times} / F^{\times}} f(\lambda)=\frac{1}{2} \sum_{\mu} \hat{f}(\mu)
$$


by the Poisson summation formula, where $\hat{f}$ is the Fourier transform of $f$ and the sum runs over all characters $\mu$ of $E_{\mathbf{A}}^{\times} / E^{\times}$with $\left.\mu\right|_{F_{\mathbf{A}}^{\times}}=\omega \tau$. (The factor $\frac{1}{2}$ arises because $\operatorname{Vol}\left(E_{\mathbf{A}}^{\times} / R_{\mathbf{A}}^{\times} E^{\times}\right)=2$ with our choice of Haar measures.) For each $\mu$ there is a unitary representation $\left(\pi_{\mu}, \mathscr{H}_{\mu}\right)$ of $G_{\mathbf{A}}$ with central character $\omega$ and an operator $A_{\mu}: \mathscr{H}_{\mu} \rightarrow \mathscr{H}_{\mu}$ such that

$$
\pi_{\mu}(g) \cdot \tau(\operatorname{det} g) A_{\mu}=\pi_{\mu}(g) A_{\mu} \quad\left(g \in G_{\mathbf{A}}\right)
$$

(these are the dihedral representations associated to the quadratic extension $E / F$ ), and $\hat{f}(\mu)=\operatorname{tr}\left(\pi_{\mu}(\varphi) \circ A_{\mu}\right)$. If $\mu=\chi \circ N_{E / F}$ for some character $\chi$ of $F$ then $\mathscr{H}_{\mu} \cong$ $\mathscr{H}_{\tau \chi, \chi, 1 / 2}$ (the " $\frac{1}{2}$ " is the value making $\mathscr{H}_{\tau \chi, \chi, s}$ unitary) and $A_{\mu}=$ $M\left(\tau \chi, \chi, \frac{1}{2}\right) \circ(\cdot \tau \circ$ det $)$. Since $\chi$ and $\tau \chi$ give the same $\mu$, we find that the right-hand side of Theorem 2 equals

$$
\frac{1}{2} \sum_{\substack{\mu \\ \mu \mid F_{\mathbf{A}}^{\times}=\omega \tau \\ \mu \neq \chi \circ N_{E / F}}} \operatorname{tr}\left(\pi_{\mu}(\varphi) \circ A_{\mu}\right),
$$

where now $\mu$ runs over characters on $E_{\mathbf{A}}^{\times} / E^{\times}$which are not of the form $\chi \circ N_{E / F}$ for any character $\chi$ of $F_{A}^{\times} / F^{\times}$, i.e. such that $\mu^{\sigma} \neq \mu(\sigma=$ nontrivial element of $\operatorname{Gal}(E / F))$. The representations $\left(\pi_{\mu}, \mathscr{H}_{\mu}\right)$ and $\left(\pi_{\mu^{\circ}}, \mathscr{H}_{\mu^{\sigma}}\right)$ are isomorphic, so this equals $\sum_{\pi} \operatorname{tr}\left(\pi(\varphi) \circ A_{\pi}\right)$, where now $\pi$ runs over all dihedral irreducible representation associated to $E$.

On the other hand, the left-hand side of the formula of Theorem 2 equals $\operatorname{tr}\left(\rho_{0}(\varphi) \circ B_{\tau}\right)$, where

$$
B_{\tau}: L_{0}^{2}\left(G_{F} \backslash G_{\mathbf{A}}, \omega\right) \rightarrow L_{0}^{2}\left(G_{F} \backslash G_{\mathbf{A}}, \omega\right)
$$

is the operation $\varphi(g) \mapsto \varphi(g) \cdot \tau(\operatorname{det} g)$, which makes sense because the restriction of $\tau$ odet to $Z_{\mathbf{A}}$ is trivial. Only those irreducible constituents $\pi$ of $L_{0}^{2}$ which are invariant under $B_{\tau}$, i.e. which satisfy $\pi=\pi \otimes \tau$, contribute to this trace, so Theorem 2 can be reexpressed as the identity

$$
\sum_{\substack{\pi \text { cuspidal } \\
\pi=\pi \otimes \tau}} \operatorname{tr}\left(\pi(\varphi) \circ B_{\tau}\right)=\sum_{\begin{array}{c}
\pi \text { irreducible } \\
\text { dihedrai representation } \\
\text { associated to } E / F
\end{array}} \operatorname{tr}\left(\pi \circ A_{\pi}\right) .
$$

This shows that the dihedral representations associated to a quadratic extension are automorphic (which had been known for a long time) and that all irreducible cuspidal representations $\pi$ with $\pi \simeq \pi \otimes \tau$ arise in this way (which is the result of Langlands-Labesse). It also shows that the operators $B_{\tau}$ and $A_{\pi}$ correspond, giving an explicit intertwining operator $\pi \stackrel{\sim}{\rightarrow} \pi \otimes \tau$.

Case 2. $\tau=1$.

This case is much harder, since all terms in the formula for $I(s)$ have poles at $s=1$ and three of them have double poles. Since the final result, the usual Selberg trace formula for GL(2), is also much better known than the result for $\tau \neq 1$, we content ourselves with a sketch of the calculation; indeed, we must confess that the computation of the residue of $I_{\chi}(s)$ is so monstrous that we have not ourselves carried it out completely. The occurrence of double poles is related to the appearance of logarithmic divergences of the individual terms when the Selberg trace 
formula is proved by the usual method of truncating the fundamental domain, as in [2]; our use of the complex parameter $s$ should be thought of as an alternative to truncation as a method of getting around the problem of the divergence of the various integrals into which $\int K_{0}(g, g) d g$ splits.

Our goal is to calculate $(2 / C) \operatorname{Res}_{s=1} I(s)$ and to show that the result is the usual trace formula, as given, e.g., in [2]. The terms $I_{E}, I_{3}, I_{4}$ and $\frac{1}{2} I_{5}$ have simple poles and easily calculated residues; the difficulty lies in the double poles of $I_{1}, I_{2}$ and $\int_{L^{\prime}} I_{\chi}(s, u) d u$. The contribution from $I_{E}$ is found as in the case $\tau=\chi_{E}$ of Case 1 to be

$$
\frac{2}{C} \operatorname{Res}_{s=1} I_{E}(s)=\int_{E_{\mathbf{A}}^{\times} \backslash G_{\mathbf{A}}} \sum_{\substack{\lambda \in E^{\times} / F^{\times} \\ \lambda \neq 1}} \varphi\left(x^{-1} \lambda x\right) d x ;
$$

adding up these contributions for all quadratic extensions $E / F$ gives the elliptic contribution (second term) in Theorem 6.33 of [2]. Applying the identity

$$
\operatorname{Res}_{s=1} \int_{\mathbf{A}^{\times}}\left(\int_{\mathbf{A}} F(u) \psi(a u) d u\right)|a|^{s} d^{\times} a=F(0) \quad(F \in S(\mathbf{A}))
$$

(§0) to $F(u)=\varphi\left[k^{-1}\left(\begin{array}{cc}1 & u \\ 0 & 1\end{array}\right) k\right]$, we find

$$
\begin{aligned}
\operatorname{Res}_{s=1} I_{3}(s) & =\varphi(1) \int_{K} f(k, 2) d k \\
& =\varphi(1) \int_{K} \int_{\mathbf{A}^{\times}} \Phi[(0, t) k]|t|^{2} d^{\times} t d k,
\end{aligned}
$$

and this equals

$$
\varphi(1) \cdot \hat{\Phi}(0) \cdot \frac{1}{2} \operatorname{Vol}\left(Z_{\mathbf{A}} G_{F} \backslash G_{\mathbf{A}}\right)
$$

by the identity at the end of $\S 0$, so the contribution of $I_{3}(s)$ to $(2 / C) \operatorname{Res}_{s=1} I(s)$ is the volume term $\varphi(1) \operatorname{Vol}\left(Z_{\mathbf{A}} G_{F} \backslash G_{\mathbf{A}}\right)$ in [2] (note that the Tamagawa number of PGL(2) equals 2). The term $I_{4}$ is a finite sum of terms like the function $\Xi(s, \chi)$ of Lemma 3 of 1.3 (but with $\varphi$ replaced by $\int_{K} f(k, s) \varphi\left(k^{-1} g k\right) d k$ ); applying the formula for $\operatorname{Res}_{s=1} \Xi(s, \chi)$ given in that lemma, we find (with $c=\frac{1}{2} \operatorname{Vol}\left(Z_{\mathbf{A}} G_{F} \backslash G_{\mathbf{A}}\right)$ as in $\S 0$ )

$$
\operatorname{Res}_{s=1} I_{4}(s)=-\frac{1}{2 c} \sum_{\chi^{2}=\omega} \int_{K} f(k, 1) \int_{Z_{\mathbf{A}} \backslash G_{\mathbf{A}}} \varphi\left(k^{-1} g k\right) \bar{\chi}(\operatorname{det} g) d g d k .
$$

Since the inner integral is independent of $k$ and (again) $\int_{K} f(k, 1) d k=C c$, we find

$$
\begin{aligned}
\frac{2}{C} \operatorname{Res}_{s=1} I_{4}(s) & =-\sum_{\chi^{2}=\omega} \int_{Z_{\mathbf{A}} \backslash G_{\mathbf{A}}} \varphi(g) \bar{\chi}(\operatorname{det} g) d g \\
& =-\sum_{\chi^{2}=\omega}\langle\varphi, \chi \circ \operatorname{det}\rangle ;
\end{aligned}
$$

this is the term $-\operatorname{tr} \rho_{\mathrm{sp}}(\varphi)$ in (6.33) of [2]. Finally,

$$
\frac{2}{C} \cdot \frac{1}{2} \operatorname{Res}_{s=1} I_{5}(s)=-\frac{1}{4} \sum_{\chi^{2}=\omega} \operatorname{tr} A_{1, \chi}(\phi)=-\frac{1}{4} \operatorname{tr}\left(M\left(\chi, \chi, \frac{1}{2}\right) \circ \pi(\phi)\right)
$$

by the same calculation as for $\tau^{2}=1, \tau \neq 1$; this is the term (6.37) in [2]. 
There remain the three terms $I_{1}, I_{2}$ and $I_{\chi}$ with double poles at $s=1$; their leading coefficients must cancel and their residues combine to give the three terms (6.34)-(6.36) of [2]. To simplify the calculation somewhat, we shall suppose that $\Phi$ is right $K$-invariant; this is no restriction since we can get the trace formula using any function $\Phi \in S\left(\mathbf{A}^{2}\right)$ with $C=\hat{\Phi}(0,0) \neq 0$, and there are certainly $K$-invariant functions with this property. We will give the proof that the double poles in $I_{1}, I_{2}$ and $\sum I_{\chi}$ cancel, and show that the contribution of $I_{1}+I_{2}$ to the residue gives (6.34) and (6.35) of [2], but, as mentioned in the introduction, we will not compute the residue of the $I_{\chi}$ terms, so that our derivation of the Selberg trace formula will be incomplete.

For any function $\phi \in \mathscr{S}(\mathbf{A})$ we define the finite part f.p. $(\phi)$ by

$$
\int_{\mathbf{A}^{\times}} \phi(a)|a|^{s} d^{\times} a=\frac{\hat{\phi}(0)}{s-1}+\text { f.p. }(\phi)+O(s-1) \quad(s \rightarrow 1)
$$

or equivalently (using the Tate integral calculations reviewed in $§ 0$ ) by

$$
\begin{aligned}
\text { f.p. }(\phi)= & \int_{|a| \geqslant T} \phi(a)|a| d^{\times} a \\
& +\int_{|a| \geqslant T^{-1}} \hat{\phi}(a) d^{\times} a-\phi(0) T+\hat{\phi}(0) \log T \quad(\text { any } T>0) \\
= & \lim _{T \rightarrow 0}\left(\int_{|a| \geqslant T} \phi(a)|a| d^{\times} a+\hat{\phi}(0) \log T\right) .
\end{aligned}
$$

It is easily checked that

$$
\text { f.p. }(a \mapsto \phi(t a))=|t|^{-1} \text { f.p. }(\phi)-\hat{\phi}(0)|t|^{-1} \log |t|
$$

for any $t \in \mathbf{A}^{\times}$. Using the finite-part functional we can give the Laurent expansion near $s=1$ of the double Tate integral $\iint \Phi(t, u)|t u|^{s} d^{\times} t d^{\times} u$ for any $\Phi \in \mathscr{S}\left(\mathbf{A}^{2}\right)$ :

$$
\begin{aligned}
& \iint_{\mathbf{A}^{\times 2}} \Phi(t, u)|t|^{s}|u|^{s} d^{\times} t d^{\times} u \\
&= \int_{\mathbf{A}^{\times}}\left(\frac{1}{s-1} \int_{\mathbf{A}} \Phi(t, u) d u+\text { f.p. }(\Phi(t, \cdot))+O(s-1)\right)|t|^{s} d^{\times} t \\
&= \frac{1}{(s-1)^{2}} \iint_{\mathbf{A}^{2}} \Phi(t, u) d t d u \\
&+\frac{1}{s-1} \text { f.p. } \cdot\left(\int_{\mathbf{A}} \Phi(t, \cdot) d t+\int_{\mathbf{A}} \Phi(\cdot, u) d u\right)+O(1) \\
&= \frac{C(\Phi)}{(s-1)^{2}}+\frac{B(\Phi)+B(w \Phi)}{s-1}+O(1)
\end{aligned}
$$

where $C(\Phi)=\hat{\Phi}(0,0)$ as before and

$$
B(\Phi)=\text { f.p. }\left(t \mapsto \int_{\mathbf{A}} \Phi(t, u) d u\right)=\text { f.p. }(\tilde{\Phi}(\cdot, 0))
$$


( $\tilde{\Phi}$ as in $\S 0$ ). If $\Phi$ is right $K$-invariant then $w \Phi=\Phi$ and we write simply $B, C$ for $B(\Phi)$ and $C(\Phi)$. An easy calculation shows that in this case $B, C$ transform by

$$
\begin{gathered}
C(g \Phi)=|\operatorname{det} g|^{-1} C(\Phi), \\
B(g \Phi)=|\operatorname{det} g|^{-1}\left(B(\Phi)-\frac{1}{2}(\log |\operatorname{det} g|+\log H(g)) C(\Phi)\right)
\end{gathered}
$$

for $g \in \mathrm{GL}(2)$, where $H(g)$ is defined by $H(g)=|a / b|$ if $g=\left(\begin{array}{cc}a & u \\ 0 & b\end{array}\right) k$ with $a$, $b \in \mathbf{A} \times, u \in \mathbf{A}, k \in K$. Hence the above formula applied to $g \Phi$ gives

$$
\begin{aligned}
|\operatorname{det} g|^{s} \iint_{\mathbf{A}^{\times 2}} \Phi[(t, u) g]|t|^{s}|u|^{s} d^{\times} t d^{\times} u \\
=\frac{C}{(s-1)^{2}}+\frac{2 B-\frac{1}{2} C \log [H(g) H(w g)]}{s-1}+O(1) .
\end{aligned}
$$

We apply this to the inner integral in the definition of $I_{1}(s)$ to get

$$
\begin{aligned}
I_{1}(s)= & \frac{1}{2} \int_{K} \int_{N_{\mathbf{A}}} \sum_{\substack{\alpha \in F^{\times} \\
\alpha \neq 1}} \phi\left[k^{-1} n^{-1}\left(\begin{array}{ll}
\alpha & 0 \\
0 & 1
\end{array}\right) n k\right] \\
& \times\left(\iint_{\mathbf{A}^{\times 2}} \Phi[(t, u) n k]|t|^{s}|u|^{s} d^{\times} t d^{\times} u\right) d n d k \\
= & \frac{1}{2} \int_{K} \int_{N_{\mathrm{A}}} \sum_{\substack{\alpha \in F^{\times} \\
\alpha \neq 1}} \phi\left[k^{-1} n^{-1}\left(\begin{array}{cc}
\alpha & 0 \\
0 & 1
\end{array}\right) n k\right] \\
& \times\left(\frac{C}{(s-1)^{2}}+\frac{2 B-\frac{1}{2} C \log H(w n k)}{s-1}+O(1)\right) d n d k \\
= & \left(\frac{\frac{1}{2} C}{(s-1)^{2}}+\frac{B}{s-1}\right) A_{1}+\frac{\frac{1}{2} C}{s-1} T_{1}+O(1)
\end{aligned}
$$

with

$$
\begin{aligned}
& A_{1}=\int_{K} \int_{\mathbf{A}} \sum_{\substack{\alpha \in F^{\times} \\
\alpha \neq 1}} \phi\left[k^{-1}\left(\begin{array}{cc}
\alpha & x \\
0 & 1
\end{array}\right) k\right] d x d k, \\
& T_{1}=-\frac{1}{2} \int_{K} \int_{N_{\mathbf{A}}} \sum_{\substack{\alpha \in F^{\times} \\
\alpha \neq 1}} \phi\left[k^{-1} n^{-1}\left(\begin{array}{cc}
\alpha & 0 \\
0 & 1
\end{array}\right) n k\right] \log H(w n k) d n d k .
\end{aligned}
$$

For $I_{2}$ the definitions of $I_{2}$ and of the finite-part functional immediately give

$$
\begin{aligned}
I_{2}(s) & =\int_{K}\left(\int_{\mathbf{A}^{\times}} \phi\left[k^{-1}\left(\begin{array}{ll}
1 & a \\
0 & 1
\end{array}\right) k\right]|a|^{s} d^{\times} a\right)\left(\frac{C}{2 s-2}+B+O(s-1)\right) d k \\
& =\left(\frac{\frac{1}{2} C}{(s-1)^{2}}+\frac{B}{s-1}\right) A_{2}+\frac{\frac{1}{2} C}{s-1} T_{2}+O(1)
\end{aligned}
$$


with

$$
\begin{gathered}
A_{2}=\int_{K} \int_{\mathbf{A}} \phi\left[k^{-1}\left(\begin{array}{cc}
1 & a \\
0 & 1
\end{array}\right) k\right] d a d k, \\
T_{2}=\text { f.p. }\left(a \mapsto \int_{K} \phi\left[k^{-1}\left(\begin{array}{ll}
1 & a \\
0 & 1
\end{array}\right) k\right] d k\right) .
\end{gathered}
$$

The expressions $T_{1}$ and $T_{2}$ are just the terms (6.35) and (6.34) in [2]. Hence to complete the proof of the trace formula we would need a formula of the form

$$
\frac{1}{2 \pi i} \int_{L^{\prime}} I_{\chi}(s, u) d u=\left(\frac{\frac{1}{2} C}{(s-1)^{2}}+\frac{B}{s-1}\right) A_{\chi}+\frac{\frac{1}{2} C}{s-1} T_{\chi}+O(1)
$$

with $\sum_{\chi} A_{\chi}=-A_{1}-A_{2}$ and $\sum_{\chi} T_{\chi}$ equal to the term (6.36) in [2]. Recall that $I_{\chi}(s, u)$ has the form

$$
I_{\chi}(s, u)=-\frac{1}{2} \sum_{\alpha, \beta \in A\left(\chi, \chi^{-1} \omega\right)}\left(\pi_{\chi, \chi^{-1} \omega, u}(\phi) f_{\beta}, f_{\alpha}\right) J_{\alpha, \beta}(s, u)
$$

where $J_{\alpha, \beta}(s, u)$ is a function whose analytic properties are given by Propositions $1-4$ of 2.5. The first of these propositions shows that $J_{\alpha, \beta}(s, u)$ is a meromorphic function of $s$ and $u$ which is regular near $s=1, u=\frac{1}{2}$ unless $\chi^{2}=\omega$, in which case it has the form

$$
J_{\alpha, \beta}(s, u)=L(s)^{2} \frac{L(s+1-2 u) L(s-1+2 u)}{L(2-2 u) L(2 u)} \times \text { regular function }
$$

$\left(L(s)=L_{F}(s, 1)\right)$. From the Taylor expansion

$$
\frac{L(s+1-2 u) L(s-1+2 u)}{L(2-2 u) L(2 u)}=1+\left(\frac{L^{\prime}}{L}(2-2 u)+\frac{L^{\prime}}{L}(s u)\right)(s-1)+O(s-1)^{2}
$$

and the fact that $\left(L^{\prime} / L\right)(2-2 u)+\left(L^{\prime} / L\right)(2 u)$ is regular at $\frac{1}{2}$, we see that $J_{\alpha, \beta}(s, u)$ has a Laurent expansion at $s=1$ of the form

$$
J_{\alpha, \beta}(s, u)=\frac{a_{\alpha \beta}(u)}{(s-1)^{2}}+\frac{b_{\alpha \beta}(u)}{s-1}+O(1)
$$

with $a_{\alpha \beta}(u)$ and $b_{\alpha \beta}(u)$ holomorphic at $u=\frac{1}{2}$. Proposition 4 of 2.5 gives the formula $a_{\alpha \beta}(u)=C \cdot \delta_{\alpha, \beta}$, and refining its proof we find that $b_{\alpha \beta}(u)$ has the form $2 B \cdot \delta_{\alpha, \beta}+C \cdot t_{\alpha, \beta}(u)$ where $t_{\alpha, \beta}(u)$ depends only on $\phi$, not on $\Phi$, and by the remarks above is regular at $u=\frac{1}{2}$. This gives a Laurent expansion of $\frac{1}{2 \pi i} \int_{L^{\prime}} I_{\chi}(s, u) d u$ of the form written above with

$$
\begin{aligned}
A_{\chi} & =-\sum_{\alpha \in A\left(\chi, \chi^{-1} \omega\right)} \frac{1}{2 \pi i} \int_{1 / 2-i \infty}^{1 / 2+i \infty}\left(\pi_{\chi, \chi^{-1} \omega, u}(\phi) f_{\alpha}, f_{\alpha}\right) d u \\
& =-\frac{1}{2 \pi i} \int_{1 / 2-i \infty}^{1 / 2+i \infty} \operatorname{tr}\left(\pi_{\chi, \chi^{-1} \omega, u}(\phi)\right) d u
\end{aligned}
$$

and

$$
T_{\chi}=-\sum_{\alpha, \beta \in A\left(\chi, \chi^{-1} \omega\right)} \frac{1}{2 \pi i} \int_{1 / 2-i \infty}^{1 / 2+i \infty}\left(\pi_{\chi \cdot \chi^{-1} \omega, u}(\phi) f_{\beta}, f_{\alpha}\right) t_{\alpha, \beta}(u) d u
$$


The formula for $\left(\pi_{\chi, \chi^{-1} \omega, u}(\phi) f_{\beta}, f_{\alpha}\right)$ at the end of 1.4 gives the identity

$$
\operatorname{tr}\left(\pi_{\chi, \chi^{-1} \omega, u}(\phi)\right)=\int_{\mathbf{A} \times} \int_{K} \int_{\mathbf{A}} \phi\left[k^{-1}\left(\begin{array}{cc}
a & x \\
0 & 1
\end{array}\right) k\right] \chi(a)|a|^{u-1} d x d k d^{\times} a
$$

and together with the Mellin inversion formula

$$
\sum_{\chi} \frac{1}{2 \pi i} \int_{1 / 2-i \infty}^{1 / 2+i \infty}\left(\int_{\mathbf{A}^{\times}} F(a) \chi(a)|a|^{u-1} d^{\times} a\right) d u=\sum_{\alpha \in F^{\times}} F(\alpha) \quad(F \in \mathscr{S}(\mathbf{A}))
$$

this shows that $A_{1}+A_{2}+\sum_{\chi} A_{\chi}$ vanishes (as indeed it must to make $(s-1) I(s)$ regular at $s=1)$. As stated previously, we will not calculate $t_{\alpha, \beta}(u)$, but only mention that the result needed to show that $\Sigma_{\chi} T_{\chi}$ agrees with the term (6.36) in [2] would be the formula

$$
t_{\alpha, \beta}(u)=\frac{1}{2}\left(M_{\chi^{-1} \omega}(u) M_{\chi}^{\prime}(u) f_{\alpha}, f_{\beta}\right),
$$

where $M_{\chi}(u)$ is defined as the composition

$$
H\left(\chi, \chi^{-1} \omega\right) \stackrel{\sim}{\rightarrow} H\left(\chi, \chi^{-1} \omega, u\right) \stackrel{M_{\chi, \chi^{-1} \omega, u}(\phi)}{\rightarrow} H\left(\chi^{-1} \omega, \chi, 1-u\right) \leftleftarrows H\left(\chi^{-1} \omega, \chi\right)
$$

and $M_{\chi}^{\prime}(u)=d M_{\chi}(u) / d u$ (we are obliged to identify all fibers of the fiber bundle $\cup_{u} H\left(\chi_{1}, \chi_{2}, u\right)$ with a fixed fiber $H\left(\chi_{1}, \chi_{2}\right)$ in order to make sense of this derivative).

3.3. On the holomorphy of certain Dirichlet series. In this section we prove

THEOREM 2. Let $\pi$ be an irreducible cuspidal representation of $G_{\mathbf{A}}$ and $\tau$ a character of $\mathbf{A}^{\times} / F^{\times}$. Then the quotient of the $L$-series $L(s, \pi \otimes \tau \times \tilde{\pi})$ by $L(s, \tau)$ is holomorphic except possibly for simple poles at $s=0$ and $s=1$ if $\tau^{2}=1, \tau \neq 1$.

As mentioned in the introduction this theorem was proved in [3] using a method introduced by Shimura to prove the same theorem in the holomorphic case [10].

We remark that the behavior near $s=1$ of $L\left(s, \pi \times \pi^{\prime}\right)$ for any pair of cuspidal representations $\pi, \pi^{\prime}$ is given by Rankin's method (see for instance [3]). In particular the poles mentioned in the theorem can only occur if $\pi \otimes \tau=\pi$, in which case $\pi$ is a dihedral representation. However, the real interest of Theorem 2 is the vanishing of $L(s, \pi \otimes \tau \times \tilde{\pi})$ at the zeroes of $L(s, \tau)$.

Proof. Let $\omega$ be a character of $\mathbf{A}^{\times} / F^{\times}$and denote by $V$ the Hilbert space $L_{0}^{2}\left(G_{F} \backslash G_{\mathbf{A}}, \omega^{-1}\right)$; then $V=\oplus_{\pi} V_{\pi}$ (Hilbert space sum) where $\pi$ varies over the set of irreducible cuspidal representations of $G_{\mathbf{A}}$ with central character $\omega^{-1}$ and $V_{\pi}$ is the corresponding isotypical component. By "multiplicity one", the representation of $G_{\mathrm{A}}$ on $V_{\pi}$ is actually equivalent to $\pi$. For each $\pi$, choose an orthonormal basis $B_{\pi}$ of $V_{\pi}$ made up of $K$-finite vectors. Let $\varphi$ as always be an element of the Hecke algebra of $G_{\mathbf{A}}$ with central character $\omega$ and $K_{0}(x, y)$ the kernel function for the action $\rho_{0}(\varphi)$ of $\varphi$ on $V$. Then we have the decomposition

$$
\begin{gathered}
K_{0}(x, y)=\sum_{\pi} K_{\pi}(x, y) \\
K_{\pi}(x, y)=\sum_{a \in B_{\pi}} \pi(\varphi) a(x) \overline{a(y)} .
\end{gathered}
$$


All of the functions occuring here are of rapid decay in $x$ and $y$. The first sum converges in $L^{2}$ and hence also in the space of rapidly decaying functions, by the usual estimates on the growth of cusp forms. The second sum is finite uniformly in $x$ and $y$ for a given $\varphi$ because of the $K$-finiteness of $\varphi$.

Proposition. Let $F(x)$ be a function on $G_{F} Z_{\mathbf{A}} \backslash G_{\mathbf{A}}$ which is $K$-finite and of slow growth (polynomial growth in a Siegel domain). Then the following statements are equivalent:

(i) $\int_{G_{F} Z_{\mathbf{A}} \backslash G_{\mathbf{A}}} K_{0}(x, x) F(x) d x=0$ for all $\varphi$;

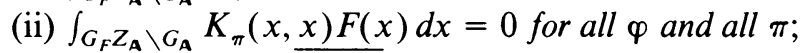

(iii) $\int_{G_{F}} Z_{\mathbf{A}} \backslash G_{\mathbf{A}} a_{1}(x) \overline{a_{2}(x)} F(x) d x=0$ for all $\pi$ and any $K$-finite functions $a_{1}$, $a_{2} \in V_{\pi}$.

To deduce the theorem from this proposition, we choose

$$
F(x)=\left.\frac{\partial^{j}}{\partial s^{j}} E\left(x, \Phi, \tau, \tau^{-1}, s\right)\right|_{s=\rho},
$$

where $\rho$ is a zero of $L(s, \tau)$ of order $n_{\rho}, j$ an integer between 0 and $n_{\rho}-1$, and $\Phi$ an arbitrary function in $\mathscr{S}\left(\mathbf{A}^{2}\right)$. The corollary to Theorem 1 (divisibility of $I(s, \varphi, \Phi, \tau)$ by $L(s, \tau))$ implies that statement (i) of the proposition holds for this $F$, and statement (iii) of the proposition implies that the function

$$
\int_{G_{F} Z_{\mathbf{A}} \backslash G_{\mathbf{A}}} a_{1}(x) \overline{a_{2}(x)} E\left(x, \Phi, \tau, \tau^{-1}, s\right) d x
$$

is divisible by $L(s, \tau)$ (except for possible poles at $s=0$ and 1) for all $a_{1}, a_{2} \in V_{\pi}$ and $\Phi \in \mathscr{S}\left(\mathbf{A}^{2}\right)$. Since the $L$-series $L(s, \pi \otimes \tau \times \tilde{\pi})$, defined as the "greatest common divisor" of these integrals, is actually the sum of a finite number of them, the theorem follows.

Proof of The Proposition. The implication (iii) $\Rightarrow$ (ii) is trivial since the sum in the formula for $K_{\pi}$ is finite. To prove the converse, choose an elementary idempotent $\xi$ such that $\pi(\xi)$ fixes $a_{1}$ and $a_{2}$. The image $V_{\xi}$ of $\pi(\xi)$ is finite-dimensional. We may assume that $B_{\pi}$ contains an orthonormal basis $B_{\pi, \xi}$ of $V_{\xi}$ and that $a_{2} \in B_{\pi, \xi}$. Let $H_{\xi}$ be the subalgebra of the Hecke algebra consisting of all $\varphi$ with $\xi * \varphi * \xi=\varphi$. Then any $\varphi \in H_{\xi}$ maps the basis vectors in $B_{\pi}-B_{\pi, \xi}$ to 0 . Since the representation of $H_{\xi}$ on $V_{\xi}$ is algebraically irreducible, we can find a $\varphi \in H_{\xi}$ which maps $a_{2}$ to $a_{1}$ and the other elements of $B_{\pi, \xi}$ to zero. Applying (ii) to this $\varphi$ gives the identity (iii).

It remains to prove that (i) implies (ii) (the converse is trivial because of the rapid convergence). Let $S$ be a finite set of places containing all infinite places. Let $H^{S}$ be the set of all functions on $G^{S}=\Pi_{v \notin S} G_{v}$ which are bi- $K$-finite and have central character $\omega$. From now on we consider only functions $\varphi$ of the form $\varphi(x)=$ $\varphi_{S}\left(x_{S}\right) \varphi^{S}\left(x^{S}\right)$ with $\varphi_{S}=\Pi_{v \in S} \varphi_{v}$ and $\varphi^{S} \in H^{S}$. Then the $K_{\pi}$ are nonzero only for those $\pi$ which contain a vector invariant under $K^{S}=\Pi_{v \notin S} K_{v}$.

Let $V_{\pi}^{S}$ be the space of $K^{S}$-invariant vectors. We can choose the basis $B_{\pi}$ such that $B_{\pi}$ contains a basis $B_{\pi}^{S}$ of $V_{\pi}^{S}$ and $H^{S}$ annihilates the vectors of $B_{\pi}-B_{\pi}^{S}$. We have an operation $\pi_{S}\left(\varphi_{S}\right)$ on $V_{\pi}^{S}$ by convolution over $G_{S}$, and for $a \in V_{\pi}^{S}$ we have

$$
\pi(\varphi) a=\lambda_{\pi}\left(\varphi^{S}\right) \cdot \pi_{S}\left(\varphi_{S}\right) a
$$


where $\lambda_{\pi}^{S}: H^{S} \rightarrow \mathrm{C}$ is an algebra homomorphism. By strong multiplicity one, the homomorphisms $\lambda_{\pi}^{S}$ are distinct for different $\pi$. Our decomposition of $K_{0}$ now becomes

$$
\begin{gathered}
K_{0}(x, y)=\sum_{\pi} \lambda_{\pi}\left(\varphi^{S}\right) K_{\pi}^{S}(x, y), \\
K_{\pi}^{S}(x, y)=\sum_{a \in B_{\pi}^{S}} \pi_{S}\left(\varphi_{S}\right) a(x) \overline{a(y)} \quad \text { (finite sum). }
\end{gathered}
$$

Then

$$
0=\int K_{0}(x, x) F(x) d x=\sum_{\pi} \lambda_{\pi}\left(\varphi^{S}\right) c_{\pi}
$$

where

$$
c_{\pi}=\int K_{\pi}^{S}(x, x) F(x) d x .
$$

Taking $\varphi^{S}=$ characteristic function of $K^{S}$, we see that $\Sigma_{\pi}\left|c_{\pi}\right|<\infty$. We now appeal to the following lemma.

Lemma. Suppose $c_{\pi}$ is a family of complex numbers with $\sum_{\pi}\left|c_{\pi}\right|<\infty$ and

$$
\sum_{\pi} c_{\pi} \lambda_{\pi}\left(\varphi^{S}\right)=0 \quad\left(\forall \varphi^{S} \in H^{S}\right) .
$$

Then $c_{\pi}=0$ for all $\pi$.

Proof (the argument we use can be found essentially in Langlands [9]). We have $H^{S}=\otimes_{v \notin S} H_{v}$ and $\lambda_{\pi}=\otimes_{v \notin S} \lambda_{\pi, v}$, where the local Hecke algebra $H_{v}$ is isomorphic to a polynomial algebra $\mathbf{C}\left[z_{v}\right]$ and the homomorphism $\lambda_{\pi, v}$ corresponds to the homomorphism $\mathbf{C}\left[z_{v}\right] \rightarrow \mathbf{C}$ obtained by specializing $z_{v}$ to a number $\theta_{\pi, v}$ which is real and bounded, $0 \leqslant \theta_{\pi, v} \leqslant C_{v}$. Indeed, the usual description of $H_{v}$ is as the set of symmetric polynomials in two variables $x_{v}$ and $y_{v}$ subject to the relation $x_{v} y_{v}=t_{v}$, where $t_{v}$ is the value of $\omega_{v}$ on a uniformizer at $v$ and is a complex number of absolute value 1 . The isomorphism $H_{v} \cong \mathbb{C}\left[z_{v}\right]$ is then obtained by mapping $z_{v}$ to $t_{v}^{-1 / 2}\left(x_{v}+y_{v}\right)$. The homomorphism $\lambda_{\pi, v}$ sends $x_{v}$ to $t_{v}^{1 / 2} q_{v}^{s}$ and $y_{v}$ to $t_{v}^{1 / 2} q_{v}^{-s}$, where, since $\pi$ is unitary, $s$ is either pure imaginary or else real and between $-\frac{1}{2}$ and $\frac{1}{2}$; thus $z_{v} \rightarrow \theta_{\pi, v}, 0 \leqslant \theta_{\pi, v} \leqslant q_{v}^{1 / 2}+q_{v}^{-1 / 2}$.

Thus each $\lambda_{\pi}$ corresponds to a point $\theta_{\pi}=\left(\theta_{\pi, v}\right)_{v \notin S}$ in the compact set $I=$ $\Pi_{v \notin S}\left[0, C_{v}\right]$, and we have the relation $\sum_{\pi} c_{\pi} f\left(\theta_{\pi}\right)=0$ for all polynomials $f$ on $I$ (by "polynomial" we of course mean polynomials in finitely many variables of the infinite product).

Now let $\pi_{1}$ be one of our representations and $\varepsilon>0$ arbitrary. Choose a finite set $T$ of representations $\pi$ such that $T \ni \pi_{1}$ and $\sum_{\pi \notin T}\left|c_{\pi}\right|<\varepsilon$. Then

$$
\left|\sum_{\pi \in T} c_{\pi} f\left(\theta_{\pi}\right)\right|=\left|-\sum_{\pi \notin T} c_{\pi} f\left(\theta_{\pi}\right)\right| \leqslant \varepsilon \max _{\theta \in I}|f(\theta)|
$$


for all polynomials $f$. By the Stone-Weierstrass theorem, this inequality holds for all continuous functions $f$ on $I$. Choose a continuous function $f \neq 0$ such that $f\left(\theta_{\pi}\right)=0$ for all $\pi$ in $T$ except $\pi_{1}$ and $\left|f\left(\theta_{\pi_{1}}\right)\right|=\max _{I}|f(\theta)|$; then the inequality above becomes $\left|c_{\pi_{1}}\right| \leqslant \varepsilon$, and since $\pi_{1}$ and $\varepsilon$ were arbitrary this proves the lemma.

\section{BIBLIOGRAPHY}

1. S. Gelbart, Automorphic forms on adele groups. Ann. of Math. Studies, No. 83, Princeton Univ. Press, Princeton, N. J., 1975.

2. S. Gelbart and H. Jacquet, Forms of GL(2) from the analytic point of view, Automorphic Forms, Representations and L-Functions, Proc. Sympos. Pure Math., vol. 33, Amer. Math. Soc., Providence, R. I., 1979, pp. 213-251.

3. $\ldots$ A relation between automorphic representations of $G L(2)$ and $G L(3)$, Ann. Sci. École Norm. Sup. (4) 11 (1978), 471-542.

4. H. Jacquet and R. P. Langlands, Automorphic forms on $G L(2)$, Lecture Notes in Math., vol. 114, Springer-Verlag, Berlin and New York, 1970.

5. H. Jacquet, Automorphic forms on GL(2). II, Lecture Notes in Math., vol. 278, Springer-Verlag, Berlin and New York, 1972.

6. D. Kazhdan, On lifting, Lie Group Representations. II, Lecture Notes in Math., vol. 1041, Springer-Verlag, Berlin and New York, 1984, pp. 209-249.

7. J. P. Labesse, L-indistinguishable representations and the trace formula for $S L(2)$, Lie Groups and their Representations, Adam Hilger, London, 1975, pp. 331-338.

8. J. P. Labesse and R. P. Langlands, L-indistinguishability for SL(2), Canad. J. Math. 31 (1979), $726-785$.

9. R. P. Langlands, Base change for GL(2), Ann. of Math. Studies, No. 96, Princeton Univ. Press, Princeton, N. J., 1980.

10. G. Shimura, On the holomorphy of certain Dirichlet series, Proc. London Math. Soc. 31 (1975), 79-98.

11. D. Zagier, Modular forms whose Fourier coefficients involve zeta-functions of quadratic fields, Modular Functions of One Variable. VI, Lecture Notes in Math., vol. 627, Springer-Verlag, Berlin and New York, 1977, pp. 105-169.

12. Eisenstein series and the Selberg trace formula. I, Automorphic Forms, Representation Theory and Arithmetic, Tata Inst. Fund. Res. Stud. Math., No. 10, Springer-Verlag, Berlin and New York, 1981.

Department of Mathematics, Columbia University, New York, New York 10027

Department of Mathematics, University of Maryland, College Park, Maryland 20742 